\title{
Two transitional type la supernovae located in the Fornax cluster member NGC 1404: SN 2007on and SN 2011iv $\star$
}

\author{
C. Gall ${ }^{1,2}$, M. D. Stritzinger ${ }^{1}$, C. Ashall ${ }^{3}$, E. Baron ${ }^{4}$, C. R. Burns ${ }^{5}$, P. Hoeflich ${ }^{6}$, E. Y. Hsiao ${ }^{6,1}$, P. A. Mazzali ${ }^{3}$, \\ M. M. Phillips ${ }^{7}$, A. V. Filippenko ${ }^{8,9}$, J. P. Anderson ${ }^{10}$, S. Benetti ${ }^{11}$, P. J. Brown ${ }^{12}$, A. Campillay ${ }^{7}$, P. Challis ${ }^{10}$, \\ C. Contreras ${ }^{7,1}$, N. Elias de la Rosa ${ }^{11}$, G. Folatelli ${ }^{12}$, R. J. Foley ${ }^{13}$, M. Fraser ${ }^{14}$, S. Holmbo ${ }^{1}$, G. H. Marion ${ }^{15}$, \\ N. Morrell ${ }^{7}$, Y.-C. Pan $^{13}$, G. Pignata ${ }^{16,17}$, N. B. Suntzeff ${ }^{12}$, F. Taddia ${ }^{18}$, S. Torres Robledo ${ }^{7}$, and S. Valenti ${ }^{19}$ \\ 1 Department of Physics and Astronomy, Aarhus University, Ny Munkegade 120, 8000 Aarhus C, Denmark \\ e-mail: christa@dark-cosmology.dk \\ 2 Dark Cosmology Centre, Niels Bohr Institute, University of Copenhagen, Juliane Maries Vej 30, 2100 Copenhagen Ø, Denmark \\ 3 Astrophysics Research Institute, Liverpool John Moores University, IC2, Liverpool Science Park, 146 Brownlow Hill, Liverpool \\ L3 5RF, UK \\ ${ }^{4}$ Homer L. Dodge Department of Physics and Astronomy, University of Oklahoma, 440 W. Brooks, Rm 100, Norman, \\ OK 73019-2061, USA \\ 5 Observatories of the Carnegie Institution for Science, 813 Santa Barbara St., Pasadena, CA 91101, USA \\ 6 Department of Physics, Florida State University, Tallahassee, FL 32306, USA \\ 7 Carnegie Observatories, Las Campanas Observatory, 601 Casilla, La Serena, Chile \\ 8 Department of Astronomy, University of California, Berkeley, CA 94720-3411, USA \\ 9 Miller Senior Fellow, Miller Institute for Basic Research in Science, University of California, Berkeley, CA 94720, USA \\ ${ }^{10}$ European Southern Observatory, Alonso de Córdova 3107, Casilla 19, Santiago, Chile \\ 11 INAF-Osservatorio Astronomico di Padova, vicolo dell Osservatorio 5, 35122 Padova, Italy \\ 12 George P. and Cynthia Woods Mitchell Institute for Fundamental Physics \& Astronomy, Texas A\&M University, Department of \\ Physics, 4242 TAMU, College Station, TX 77843, USA \\ 13 Department of Astronomy and Astrophysics, University of California, Santa Cruz, CA 95064, USA \\ 14 School of Physics, O’Brien Centre for Science North, University College Dublin, Belfield, Dublin 4, Ireland \\ 15 Department of Astronomy, University of Texas, Austin, TX 78712, USA \\ 16 Departamento de Ciencias Fisicas, Universidad Andres Bello, Avda. Republica 252, Santiago, Chile \\ 17 Millennium Institute of Astrophysics, 782-0436 Macul, Santiago, Chile \\ 18 The Oskar Klein Centre, Department of Astronomy, Stockholm University, AlbaNova, 10691 Stockholm, Sweden \\ 19 Department of Physics, University of California, Davis, CA 95616, USA
}

Received 29 March 2017 / Accepted 19 September 2017

\begin{abstract}
We present an analysis of ultraviolet (UV) to near-infrared observations of the fast-declining Type Ia supernovae (SNe Ia) 2007on and 2011 iv, hosted by the Fornax cluster member NGC 1404. The $B$-band light curves of SN 2007on and SN 2011iv are characterised by $\Delta m_{15}(B)$ decline-rate values of $1.96 \mathrm{mag}$ and $1.77 \mathrm{mag}$, respectively. Although they have similar decline rates, their peak $B$ - and $H$-band magnitudes differ by $\sim 0.60 \mathrm{mag}$ and $\sim 0.35 \mathrm{mag}$, respectively. After correcting for the luminosity vs. decline rate and the luminosity vs. colour relations, the peak $B$-band and $H$-band light curves provide distances that differ by $\sim 14 \%$ and $\sim 9 \%$, respectively. These findings serve as a cautionary tale for the use of transitional SNe Ia located in early-type hosts in the quest to measure cosmological parameters. Interestingly, even though SN 2011iv is brighter and bluer at early times, by three weeks past maximum and extending over several months, its $B-V$ colour is 0.12 mag redder than that of SN 2007on. To reconcile this unusual behaviour, we turn to guidance from a suite of spherical one-dimensional Chandrasekhar-mass delayed-detonation explosion models. In this context, ${ }^{56} \mathrm{Ni}$ production depends on both the so-called transition density and the central density of the progenitor white dwarf. To first order, the transition density drives the luminosity-width relation, while the central density is an important second-order parameter. Within this context, the differences in the $B-V$ colour evolution along the Lira regime suggest that the progenitor of SN 2011iv had a higher central density than SN 2007on.
\end{abstract}

Key words. supernovae: general - supernovae: individual: SN 2007on - supernovae: individual: SN 2011iv - dust, extinction

\section{Introduction}

Type Ia supernovae are fundamental extragalactic distance indicators used to map out the expansion history of the Uni-

\footnotetext{
* The photometry tables are only available at the CDS via anonymous ftp to cdsarc.u-strasbg. fr (130.79.128.5) or via http://cdsarc.u-strasbg.fr/viz-bin/qcat?]/A+A/611/A58
}

verse. In doing so, they provide an accurate estimate of the Hubble constant (Riess et al. 2016) and a means to study the nature of dark energy (e.g. Suzuki et al. 2012; Betoule et al. 2014; Narayan et al. 2016). Although details of their origin remain a mystery, SNe Ia have long been considered to arise from the thermonuclear disruption of carbon-oxygen white dwarfs in binary star systems (Hoyle \& Fowler 1960). 
Today, SN Ia cosmology is no longer limited by sample size but rather by a subtle matrix of systematic errors. In order for future SN Ia experiments to differentiate between various static and or time-dependent types of dark energy, models will require an improvement in the precision of SN Ia peak luminosity measurements to $\sim 1 \%$ out to $z \approx 1.0$ (Albrecht et al. 2006). Contemporary SN Ia experiments achieve a precision of $\sim 5 \%$ (Barone-Nugent et al. 2012; Kelly et al. 2015). To reach percent-level distances will require a rest-frame near-infrared (NIR) SN Ia sample located in the Hubble flow where relative peculiar motions are small (Folatelli et al. 2010; Mandel et al. 2011), along with improved photometric calibrations and (ultimately) a deeper theoretical understanding of the progenitors and explosion mechanism than currently exists.

Current efforts to improve upon the most dominant systematics are focusing on the construction of homogeneous samples of low-redshift SNe Ia (e.g. Hamuy et al. 2006; Ganeshalingam et al. 2010; Stritzinger et al. 2011b), improvements in photometric calibration techniques (e.g. Rheault et al. 2010; Stubbs \& Brown 2015), and an expanded understanding of SN Ia intrinsic colours and dust corrections (e.g. Folatelli et al. 2010; Mandel et al. 2011; Burns et al. 2014). Additional efforts to further understand SN Ia progenitor systems and their explosion physics are being made through, amongst others, focused studies of their ultraviolet (UV; e.g. Foley et al. 2016) and NIR properties (e.g. Hsiao et al. 2013; Diamond et al. 2015), as well as studies probing their immediate circumstellar environments (e.g. Sternberg et al. 2011; Pan et al. 2015).

Over the past $30 \mathrm{yrs}$, significant observational efforts have revealed the existence of multiple subclasses of SNe Ia. The first clear indication of departure from homogeneity arrived with the study of the low-luminosity SN 1986G, located in Centaurus A (e.g. Branch 1987; Phillips et al. 1987; Ashall et al. 2016b). Five years later, with the discovery of both the high-luminosity SN 1991T (Filippenko et al. 1992b; Ruiz-Lapuente et al. 1992; Phillips et al. 1992) and the low-luminosity SN 1991bg (Filippenko et al. 1992a; Leibundgut et al. 1993; Turatto et al. 1996), it became obvious that SNe Ia exhibit significant diversity (see, e.g. Filippenko 1997).

At the faint end of the SN Ia luminosity distribution, one finds subtypes including the intrinsically red and subluminous SN 1991bg-like SNe Ia (Filippenko et al. 1992a; Leibundgut et al. 1993; Turatto et al. 1996) and the so-called "transitional" objects. The light curves of SN 1991bg-like SNe Ia decline rapidly and are characterised by NIR light curves that exhibit a single maximum after the optical peak magnitudes are reached. In contrast, the transitional subtypes have NIR light curves exhibiting two maxima, similar to those of normal SNe Ia, with their first maximum being reached before the peak of the optical bands. Additionally, they are brighter than the SN 1991bg-like SNe Ia, despite having similar $\Delta m_{15}(B)$ values of 1.7-2.0 mag (see, e.g. Ashall et al. 2016a). Examples of transitional SNe Ia include SN 2003hv (Leloudas et al. 2009), iPTF 13ebh (Hsiao et al. 2015), and SN 2015bp (Srivastav et al. 2017).

In this paper, we focus on the Type Ia SN 2007on and SN 2011iv, both located in the Fornax cluster member NGC 1404. Within the context of the light-curve luminosity vs. width relation characterised by the light-curve decline-rate parameter $\Delta m_{15}(B)$ (Phillips 1993), these two objects are located at the faint end of the SN Ia luminosity distribution. However, with $\Delta m_{15}(B)$ measured values of $\sim 1.7-2.0 \mathrm{mag}$, along with a number of other photometric and spectroscopic properties as discussed below, SN 2007on and SN 2011iv are most akin to

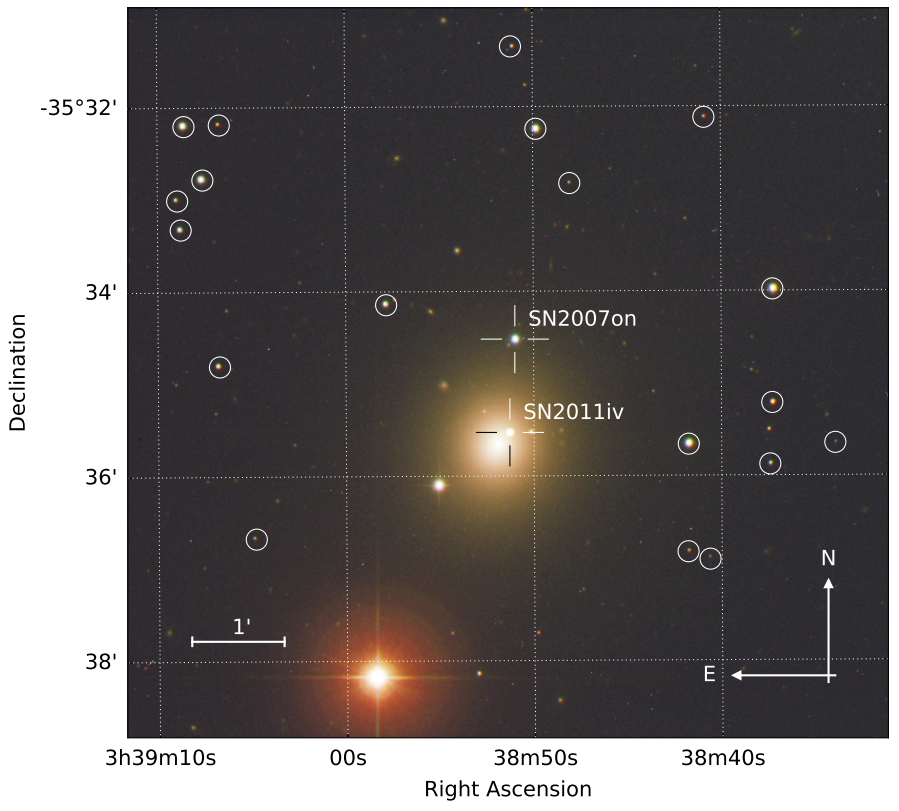

Fig. 1. Composite image of NGC 1404 with the positions of SN 2007on, SN 2011iv, and a number of local sequences stars indicated.

the transitional SN Ia iPTF 13ebh (Hsiao et al. 2015). As both SN 2007on and SN 2011iv were hosted by the same galaxy, they offer a rare opportunity to test the precision of transitional SNe Ia as distance indicators.

Here, comprehensive observations (Sect. 2) of SN 2007on and SN 2011iv are presented and studied with the intent to gain a better understanding on the nature of transitional SNe Ia and their progenitors. In Sect. 3, a detailed analysis of the photometric dataset is presented, including a study of their light-curve behaviour, their $B-V$ colour evolution, estimates of their hostgalaxy reddening, as well as their ${ }^{56} \mathrm{Ni}$ masses derived from constructed bolometric (UV, optical, and IR, hereafter UVOIR) light curves. In addition, given that both objects are very similar and located in the same galaxy, they provide an excellent opportunity to test the methods used to estimate their distance (Sect. 4). A detailed spectral analysis including modelling of the optical maximum-light spectra of both $\mathrm{SNe}$ Ia is then presented in Sect. 5, followed by a discussion in Sect. 6. Our conclusions are summarised in Sect. 7.

SN 2007on and SN 2011iv occurred in the Fornax cluster member and early-type (E1) elliptical galaxy NGC 1404 (Corwin et al. 1994), and their location along with a number of local sequence stars is presented in Fig. 1. Both SNe Ia are northwest of the centre of NGC 1404 within the projected bow-shock region of the galaxy, which itself is on an infall course directed toward the central galaxy of the Fornax cluster, NGC 1399 (e.g. Machacek et al. 2005). Interestingly, NGC 1404 has the lowest dust to stellar flux ratio of galaxies in the KINGFISH survey (Skibba et al. 2011), suggesting the absence of significant amounts of dust.

SN 2007on was discovered on 5.25 November 2007 UT (Pollas \& Klotz 2007) and identified as a subluminous, fastdeclining SN Ia (Gal-Yam et al. 2007; Morrell et al. 2007). An X-ray source close to the position of SN 2007on identified in archival Chandra images was suggested, but not unambiguously confirmed, to be the progenitor of SN 2007on (Voss \& Nelemans 2008; Roelofs et al. 2008).

Four years after the discovery of SN 2007on, the Type Ia SN 2011iv was discovered in NGC 1404 on 2.57 December 
2011 UT (Drescher et al. 2011). At a projected radius of $\lesssim 2 \mathrm{kpc}$ from the host's centre, its location is close to the effective radius of the galaxy (Loewenstein et al. 1994), unlike SN 2007on, which is at a projected radius of about $8 \mathrm{kpc}$ from the centre. Visual-wavelength spectroscopy indicated that SN 2011iv was a young, rising SN Ia (Noguchi et al. 2011; Stritzinger et al. 2011a).

There are numerous direct distance measurements obtained with various different methods published in the literature and compiled on NED ${ }^{1}$ for NGC 1404 . This includes surface brightness fluctuation (SBF) distances ranging from about $15.7 \mathrm{Mpc}$ up to $22.2 \mathrm{Mpc}$ (e.g. Tonry 1991; Blakeslee et al. 2009; Liu et al. 2002); as well as among others: fundamental plane, planetary nebula luminosity function, and Tully Fisher distance estimates (see NED). Here we adopt a distance of $17.9 \pm 2.9 \mathrm{Mpc}$ (corresponding to a distance modulus of $\mu=31.27 \pm 0.20 \mathrm{mag}$ ), which is consistent with the Advanced Camera for Surveys (ACSs) Fornax Cluster Survey estimate based on the half-light radii of globular clusters (Masters et al. 2010), though with a more conservative uncertainty.

\section{Observations}

Detailed optical and NIR light curves of SN 2007on obtained by the first phase of the Carnegie Supernova Project (CSP-I, 2004-2009; Hamuy et al. 2006) were published by Stritzinger et al. (2011b), while visual-wavelength spectra obtained at early and late times are presented by Folatelli et al. (2013) and Maeda et al. (2010). Here we complement these observations with two previously unpublished visual-wavelength spectra at $+11 \mathrm{~d}$ and $+73 \mathrm{~d}$ obtained with the ESO-NTT (+ EMMI: ESO Multi-Mode Instrument) and two NIR spectra with the ESO-NTT (+ SOFI: Son of ISAAC). Furthermore, we present recalibrations of the spectra and updated photometry of SN 2007on computed using a more accurate measurement of the local-sequence photometry, as well a vastly improved reduction of the late-phase Gemini-South spectrum of SN 2007on.

Over the course of a second instalment of the CSP, referred to as CSP-II (2011-2015), detailed optical and NIR follow-up imaging and spectroscopy were obtained for SN 2011iv, extending from $-9 \mathrm{~d}$ to $+260 \mathrm{~d}$ relative to the epoch of $B$-band maximum. Combining the CSP-II observations with five epochs of UV-optical spectroscopy obtained with the UltraViolet Optical Telescope (UVOT) aboard the Swift satellite (Burrows et al. 2005; Roming et al. 2005) and seven epochs of Hubble Space Telescope (HST) (+ STIS: Space Telescope Imaging Spectrograph) UV and visual-wavelength spectroscopy, and a large number of additional visual-wavelength and NIR spectra obtained through various facilities, yields the most detailed datasets yet obtained for a transitional SN Ia.

\subsection{Ultraviolet, optical and NIR imaging}

UV uvw2-, uvm2-, and uvw1-band imaging of both SN 2007on and SN 2011iv were obtained with Swift (+ UVOT). Photometry of SN 2007on and SN 2011iv was computed following the method described in detail by Brown et al. (2014), who use the calibration published by Breeveld et al. (2011). The Swift UVOT images and photometry are also available as part of the Swift Optical Ultraviolet Supernova Archive (SOUSA; Brown et al. 2014). In the process of computing definitive photometry, science images of SN 2011 iv were used for host-galaxy

\footnotetext{
NED is the NASA/IPAC Extragalactic Database (Steer et al. 2017).
}

subtraction of SN 2007on's science images, while science images of SN 2007on were used for host-galaxy subtraction of SN 2011iv's science images. Definitive UVOT uvw2-, uvm2-, and $u v w 1$-band photometry is provided in Table F.1; we note that the photometry of SN 2007on presented here supersedes the photometry published by Milne et al. (2010).

Optical ugriBV-band imaging of SN 2007on and SN 2011iv was obtained with the Henrietta Swope $1.0 \mathrm{~m}$ telescope (+ SITe3 direct CCD camera) located at the Las Campanas Observatory (LCO). The NIR YJH-band imaging of SN 2007on was obtained with the Swope (+ RetroCam) and the Irénée du Pont $2.5 \mathrm{~m}$ (+ WIRC: Wide Field Infrared Camera) telescopes (Stritzinger et al. 2011b), while in the case of SN 2011iv all NIR $Y J H$-band imaging was taken with RetroCam attached to the Irénée du Pont telescope.

The reduction procedure applied to all imaging data is described in detail in the final CSP-I SN Ia data release (Krisciunas et al., in prep.). In brief, point-spread function (PSF) photometry is computed differentially to a local sequence of stars in the field of NGC 1404. The optical local sequence is calibrated relative to Landolt (1992) (BV) and Smith et al. (2002) (ugri) standardstar fields observed over multiple photometric nights. The NIR $J$-band and $H$-band local sequences were calibrated relative to the Persson et al. (1998) standard stars, while the $Y$-band local sequence was calibrated relative to standard $Y$-band magnitudes computed using a combination of stellar atmosphere models (Castelli \& Kurucz 2003) with the $J-K_{\mathrm{s}}$ colours of the Persson et al. standard-star catalogue (Hamuy et al. 2006).

Absolute optical photometry in the "standard" photometric system and NIR photometry in the "natural" photometric system of the local sequences used to compute photometry of SN 2007on and SN 2011iv are provided in Tables F.2 and F.3, respectively. The accompanying uncertainties corresponding to the weighted average of the instrumental errors obtained over the various nights in which photometric standard fields were observed.

Prior to computing photometry of the SNe Ia, host-galaxy template subtraction was performed on each science image. Deep host-galaxy template images were obtained with the du Pont telescope under excellent seeing conditions well after the SNe Ia faded beyond the detection limit. With galaxy-subtracted science images in hand, final optical and NIR photometry of SN 2007on and SN 2011iv was computed on the CSP "natural" system. The optical and NIR photometry of SN 2007on and SN 2011 iv is provided in Tables F.4 and F.5, respectively ${ }^{2}$. The associated uncertainty of each photometric data point is computed by adding in quadrature of the instrumental error and the nightly zero-point error. Final Swift UV and CSP optical and NIR light curves of SN 2007on and SN 2011iv are presented in Fig. 2, including best-fit template light curves computed by SNooPy (Burns et al. 2011).

\subsection{Ultraviolet, visual-wavelength, and NIR spectroscopic observations}

Multiple epochs of spectroscopy were obtained of SN 2007on and SN 2011iv with a variety of facilities; details summarising these observations are provided in Tables F.6 and F.7, respectively.

\footnotetext{
2 Definitive photometry of the local sequence stars and the SNe Ia is also available electronically on the CSP webpage http://csp.obs. carnegiescience.edu/data/
} 


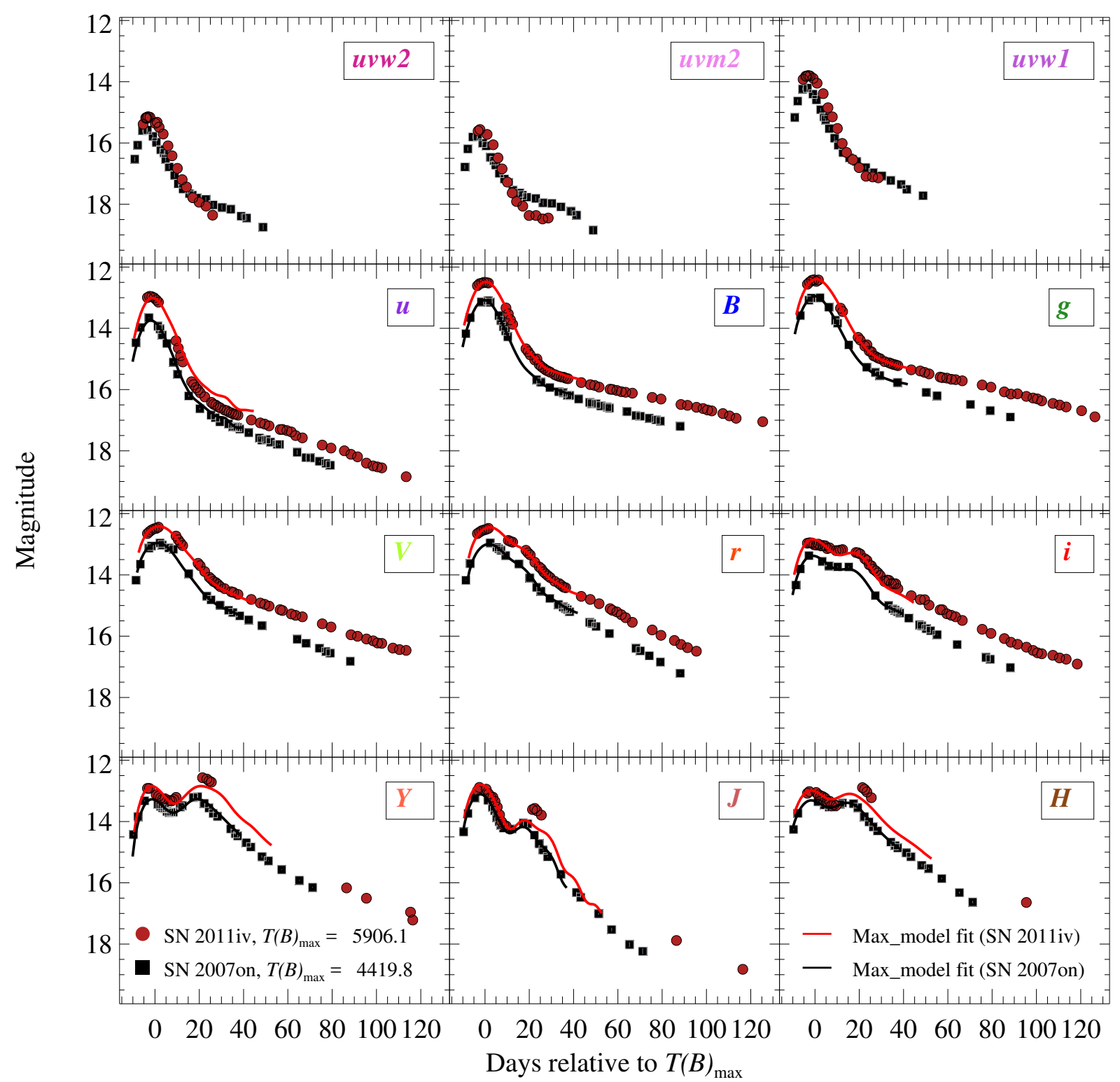

Fig. 2. UV, optical, and NIR light curves of SN 2007on (squares) and SN 2011iv (circles). The black and red solid lines represent the best SNooPy "max model" fits.

The spectroscopic time-series of SN 2007on consists of 23 epochs ranging from $-4.0 \mathrm{~d}$ to $+380 \mathrm{~d}$. The early-phase spectra were published by Folatelli et al. (2013), while Maeda et al. (2010) presented the two oldest nebular-phase spectra. In addition to these, presented here for the first time are two additional epochs of spectra obtained with the NTT (+ EMMI). These observations were conducted between $+12 \mathrm{~d}$ and $+74 \mathrm{~d}$, and the data were reduced following standard procedures within the $\operatorname{IRAF}^{3}$ environment. Additionally, we present two epochs of NIR spectroscopy also obtained with the NTT (+ SOFI), and these data were reduced as described by Smartt et al. (2015).

In the case of SN 2011iv, a total of 23 epochs of visualwavelength spectroscopy was obtained between $-7 \mathrm{~d}$ and +276 d. In addition, twelve epochs of UV spectroscopy were procured, yielding one of the most comprehensive UV datasets

3 IRAF is distributed by the National Optical Astronomy Observatories, which are operated by the Association of Universities for Research in Astronomy, Inc., under cooperative agreement with the US National Science Foundation. yet obtained for a fast-declining SN Ia. This includes five epochs $(-5 \mathrm{~d}$ to $+1 \mathrm{~d})$ taken with Swift (+UVOT; wavelength range $0.19-0.68 \mu \mathrm{m}$ ) and seven epochs (ranging from $-0.4 \mathrm{~d}$ to $+29 \mathrm{~d}$ ) taken with HST (+ STIS; wavelength range 0.18-1.023 $\mu \mathrm{m}$; GO-12592). Additionally, 16 epochs of NIR spectroscopy of SN 2011iv were obtained between $-2 \mathrm{~d}$ and $+141 \mathrm{~d}$.

Spectral data were reduced in the standard manner. Swift spectra were reduced as described by Pan et al. (in prep.), HST data reductions follow the prescription of Foley et al. (2012b), and the ground-based visual-wavelength data were reduced following the methods described by Hamuy et al. (2006). NIR spectroscopy obtained by the Magellan $6.5 \mathrm{~m}$ Baade telescope (+ FIRE; Folded Port Infrared Echellette) was reduced using the FIREHORSE software package developed by Rob Simcoe (see Hsiao et al. 2013), while data taken with the VLT (+ ISAAC: Infrared Spectrometer And Array Camera) and the NTT (+ SOFI) were reduced as described by Smartt et al. (2015).

The UV spectroscopic time-series of SN 2011iv is plotted in Fig. 3 and the visual-wavelength time-series of SN 2007on 

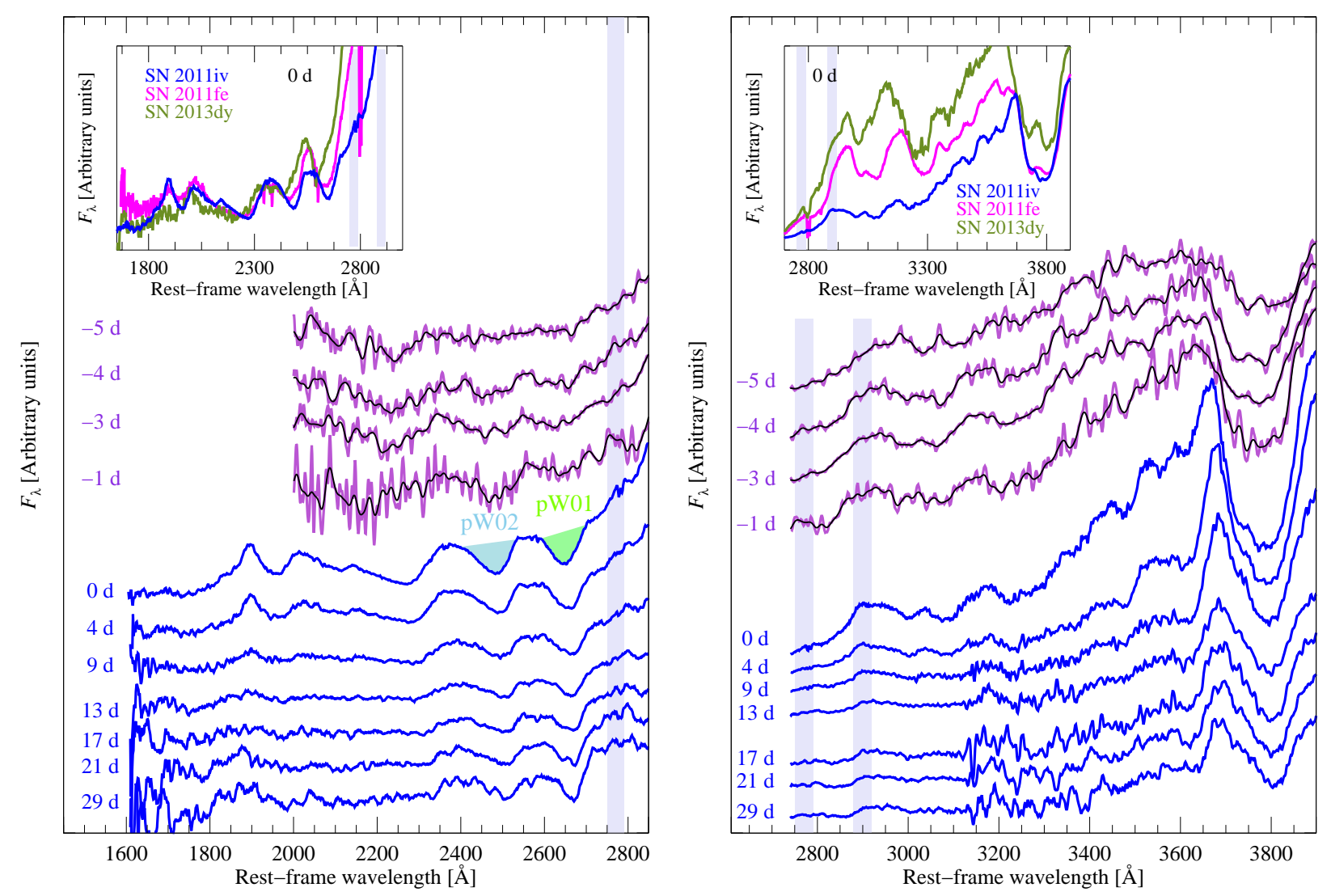

Fig. 3. Time-series of UV-wavelength spectroscopy of SN 2011iv obtained with Swift (purple) and HST (blue) over the course of nearly a month beginning $5 \mathrm{~d}$ before maximum brightness. Smoothed versions of the Swift spectra are presented in black. The insets are close-up views of the

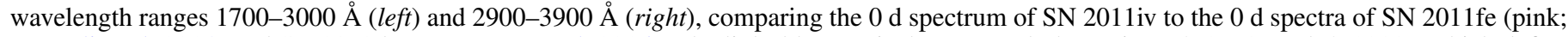
Mazzali et al. 2014) and SN 2013dy (green; Pan et al. 2015). The light-blue vertical areas mark the regions $f_{\lambda}(2770)$ and $f_{\lambda}(2900)$, which define the UV ratio $\mathcal{R}_{\mathrm{UV}}$ (see Appendix A). The green and blue shaded regions indicate the area enclosed by the pseudo-equivalent widths defined as pW01 and pW02, respectively (Sect. 5.1.2).

and SN 2011iv are presented in Fig. 4. Figure 5 displays the NIR spectra of SN 2011iv, as well as a spectrum of SN 2007on. Finally, the late-phase visual-wavelength spectra of both SNe Ia are presented in Fig. 6.

\section{Photometric analysis}

\subsection{Light curves}

The optical light curves of both objects are well sampled around the primary maximum, and high-cadence photometry extends to $\sim+80 \mathrm{~d}$ for SN 2007on and $\sim 120 \mathrm{~d}$ for SN 2011iv. The NIR light curves of SN 2007on are also densely sampled within the first three months of its evolution. However, the NIR light curves of SN 2011iv mainly cover the rise and fall around the primary (peak) maximum, with the secondary maximum only partially covered within a few days of its peak value. Additionally, individual NIR photometric points were obtained between $+80 \mathrm{~d}$ and $+120 \mathrm{~d}$.

The optical light curves of SN 2007on and SN 2011iv exhibit, compared to normal $\mathrm{SNe} I a$, a relatively quick decline from maximum light. In the case of the riYJH-band light curves, a transition to a secondary maximum around $20 \mathrm{~d}$ past the primary maximum is apparent, which is followed by a linear decline in brightness extending to late phases (e.g. Leloudas et al. 2009). For both $\mathrm{SNe} \mathrm{Ia}$, the $H$-band secondary maxima are nearly equal to the luminosity of the primary maxima, while the $Y$-band secondary maxima exceed the brightness of the primary maxima. In
Fig. 2, the light-curve shapes for the two SNe Ia are very similar, yet curiously, SN 2011iv is brighter than SN 2007on in all passbands. The observed peak brightness difference is found to decline gradually as a function of wavelength from $\sim 0.6$ mag in the $B$ band to $\sim 0.35 \mathrm{mag}$ in the $H$ band.

Estimates of key light-curve parameters are computed using the SN Ia template light-curve fitting package SNooPy (Burns et al. 2011). SNooPy offers several different models to fit SN Ia light curves, and the most appropriate model to use depends on the particular question being addressed. To estimate the time of maximum brightness, $T_{\max }$, the peak magnitude in each passband, $m_{X}$ (where $X=u, g, r, i, B, V, Y, J, H$ corrected for time dilation, Galactic reddening, and $K$ corrections) we use SNooPy's “max model” (see Stritzinger et al. 2010, Eq. (5)), adopting the colour-stretch parameter, $s_{B V}{ }^{4}$. For reasons outlined by Burns et al. (2014), $s_{B V}$ is preferred over $\Delta m_{15}$ to obtain the aforementioned light-curve parameters in the case of fastdeclining SNe Ia. The results are summarised in Table 1.

Figure 2 compares the resulting best template light-curve fits to the optical and NIR light curves. With an $s_{B V}$ value of $0.57 \pm$ 0.04 for SN 2007on and $0.64 \pm 0.04$ for SN 2011iv, both objects are found to evolve photometrically like fast-declining $\mathrm{SNe}$ Ia (i.e. $s_{B V} \lesssim 0.7$ or $\Delta m_{15} \gtrsim 1.7 \mathrm{mag}$ ). This is confirmed from

4 Recently introduced by Burns et al. (2014), the colour-stretch parameter, $s_{B V}$, is a dimensionless parameter defined to be the epoch after maximum light when the $B-V$ colour reaches its maximum value, divided by 30 days. 

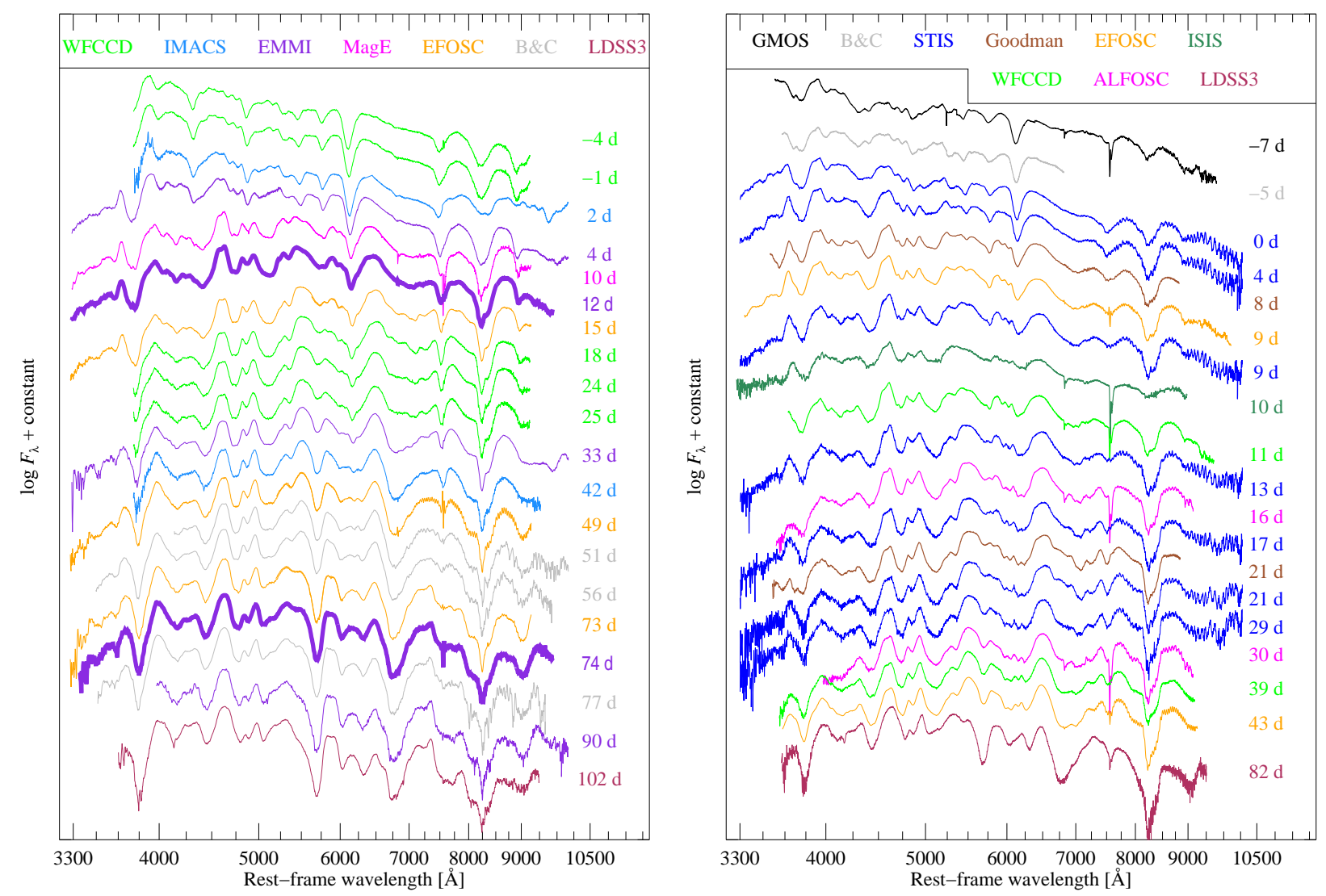

Fig. 4. Montage of selected visual-wavelength spectra of SN 2007on (left) and SN 2011iv (right). The spectra are colour coded with respect to the facility used to obtain the observations. Previously unpublished NTT (+EMMI) spectra of SN 2007on are highlighted as thick purple lines. All spectra are listed in Tables F.6 and F.7.

Table 1. Light curve parameters of SN 2007on and SN 2011iv.

\begin{tabular}{lrrc}
\hline \hline Parameter & SN 2007on & SN 2011iv & Unit \\
\hline$T(B)_{\max }{ }^{a}$ & $2454419.8 \pm 0.04$ & $2455906.1 \pm 0.05$ & JD \\
$s_{B V}{ }^{a}{ }_{u}^{a b}$ & $0.57 \pm 0.03$ & $0.64 \pm 0.03$ & \\
$m_{B}$ & $13.62 \pm 0.01$ & $12.91 \pm 0.01$ & $\mathrm{mag}$ \\
$m_{B}$ & $13.03 \pm 0.01$ & $12.44 \pm 0.01$ & $\mathrm{mag}$ \\
$m_{g}$ & $12.92 \pm 0.01$ & $12.37 \pm 0.01$ & $\mathrm{mag}$ \\
$m_{V}$ & $12.92 \pm 0.01$ & $12.38 \pm 0.01$ & $\mathrm{mag}$ \\
$m_{r}$ & $12.98 \pm 0.01$ & $12.43 \pm 0.01$ & $\mathrm{mag}$ \\
$m_{i}$ & $13.35 \pm 0.02$ & $12.82 \pm 0.01$ & $\mathrm{mag}$ \\
$m_{Y}$ & $13.26 \pm 0.01$ & $12.83 \pm 0.07$ & $\mathrm{mag}$ \\
$m_{J}$ & $13.10 \pm 0.02$ & $12.76 \pm 0.03$ & $\mathrm{mag}$ \\
$m_{H}$ & $13.25 \pm 0.02$ & $12.87 \pm 0.05$ & $\mathrm{mag}$ \\
\hline$E(B-V)_{\text {host }}{ }^{c}$ & $-0.052 \pm 0.07$ & $-0.07 \pm 0.07$ & $\mathrm{mag}$ \\
$E(B-V)_{\text {lira }}{ }^{c}$ & $-0.025 \pm 0.07$ & $0.10 \pm 0.07$ & $\mathrm{mag}$ \\
$\mu_{\mathrm{SNooPy}}{ }^{e}$ & $31.57 \pm 0.09$ & $31.17 \pm 0.09$ & $\mathrm{mag}$ \\
$\Delta m_{15}(B)^{e}$ & $1.96 \pm 0.01$ & $1.77 \pm 0.01$ & $\mathrm{mag}$ \\
\hline
\end{tabular}

Notes. ${ }^{(a)}$ Based on SNooPy fits using the max model $s_{B V}$ parameterization. ${ }^{(b)}$ Peak magnitudes estimated from photometry $K$-corrected using the Hsiao et al. (2007) spectral template, and corrected for Galactic extinction. ${ }^{(c)}$ Based on SNooPy fits using the "EBV_method2" model. The reported uncertainty was determined by adding in quadrature both statistical and systematic errors. ${ }^{(d)}$ Based on the Lira-relation fits (cf. Folatelli et al. 2010). ${ }^{(e)}$ Based on direct "Gaussian process" spline fits computed within the SNooPy envirnoment.

direct Gaussian process spline fits to the $B$-band light curves of each object, yielding $\Delta m_{15}(B)$ values of $1.96 \pm 0.01 \mathrm{mag}$ for SN 2007on and $1.77 \pm 0.01 \mathrm{mag}$ for SN 2011iv. The measured light-curve parameters for SN 2007on and SN 2011iv compare well with other works in the literature (e.g. Stritzinger et al. 2011b; Foley et al. 2012b; Burns et al. 2014); although specific light-curve parameter values vary, both $\mathrm{SNe}$ Ia are undoubtedly identified as subluminous.

Examination of the NIR light curves of both SNe Ia reveals that they peak prior to the epoch of $B$-band maximum. This, together with the presence of a secondary maximum, are key photometric characteristics of a normal SN Ia (Krisciunas et al. 2009). In contrast, fast-declining SN 1991bg-like SNe Ia only exhibit a single NIR maximum that peaks after the time of $B$-band maximum. In summary, the measured $s_{B V}$ values and the spectral characteristics discussed below, indicate SN 2007on and SN 2011iv are both similar to SN 2003gs (Krisciunas et al. 2009), SN 2003hv (Leloudas et al. 2009), and iPTF 13ebh (Hsiao et al. 2015), all of which are transitional SNe Ia.

Figure 7 displays the luminosity vs. decline-rate relation parametrised by $\Delta m_{15}$ (left) and $s_{B V}$ (right) for an extended sample of SNe Ia observed by the CSP-I (e.g. Contreras et al. 2010; Stritzinger et al. 2011b) as well as the fast-declining SN 1986G (Phillips et al. 1987) and SN 1991bg (Filippenko et al. 1992a; Leibundgut et al. 1993; Turatto et al. 1996). Both SN 2007on and SN 2011iv are brighter than expected for typical fastdeclining $\mathrm{SNe}$ Ia and are therefore located closer to the faint end of the luminosity decline-rate relation of normal SNe Ia.

\section{2. $B-V$ and ultraviolet colour evolution}

Figure 8 compares the $B-V$ colour evolution of SN 2007on and SN 2011iv to that of the normal SN 2006dd and the 


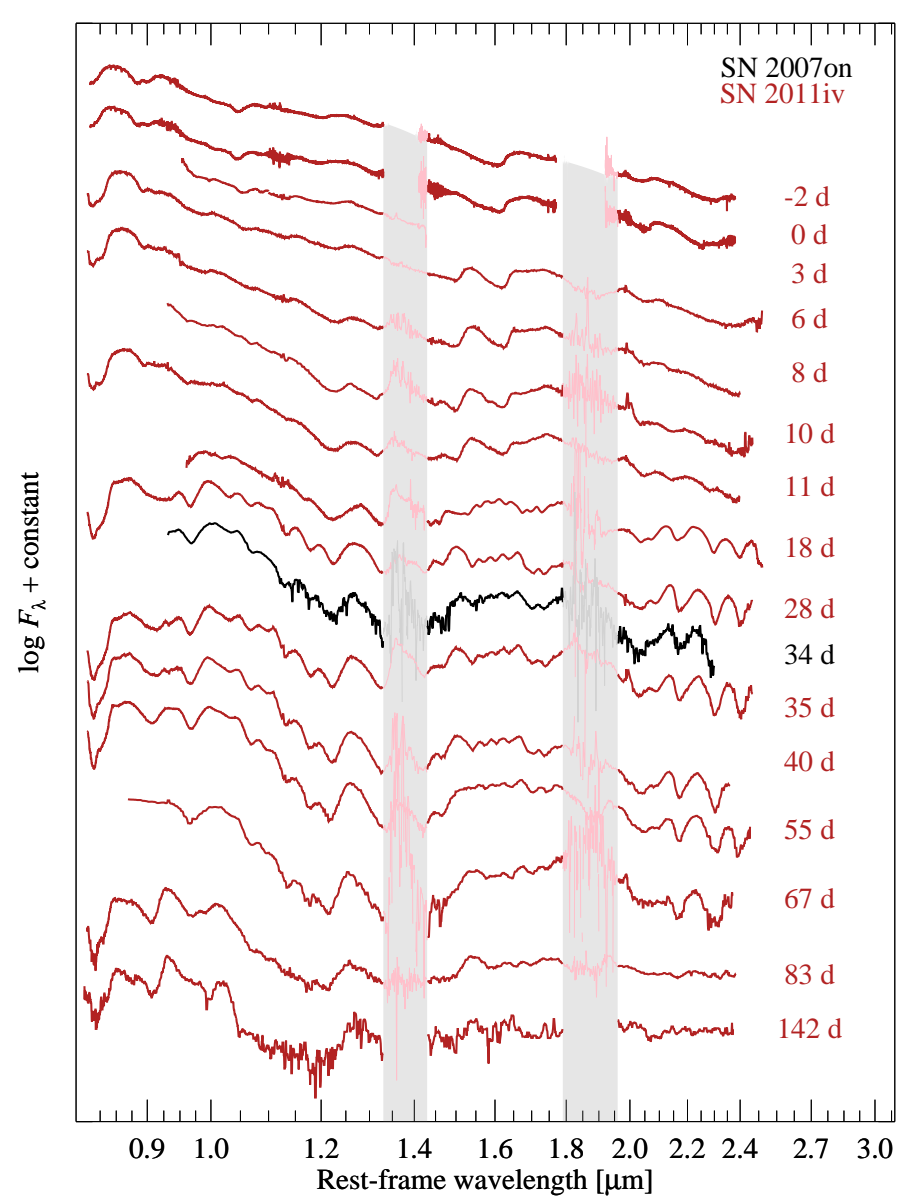

Fig. 5. Selected NIR-wavelength spectra of SN 2007on (black) and SN 2011iv (red) taken between $-2 \mathrm{~d}$ and $+142 \mathrm{~d}$ with various instruments (see Tables F.6 and F.7). The grey vertical bands indicate regions of prevalent telluric absorption. Some spectra have been smoothed for presentation purposes.

SN 1991bg-like SN 2006mr (both hosted in Fornax A; see Sect. 1), and the transitional SN Ia iPTF13ebh (Hsiao et al. 2015). The comparison SNe Ia act as representatives for each SN Ia subgroup, and are chosen because of either their close location (member of the Fornax cluster) or their excellent data coverage.

While overall the $B-V$ colour evolution of all of these objects follows a very similar morphology, there are differences in the $B-V$ colour values apparent for the different types of $\mathrm{SNe}$ Ia. Typically, at early epochs (before $B$-band maximum), the $B-V$ colours have their bluest values. However, as the light curves evolve past maximum, the colour values abruptly become redder again, successively reaching their maximum over a period of $10 \mathrm{~d}$ (in the case of SN 2006mr) to $20 \mathrm{~d}$ for the other SNe Ia. Thereafter, the $B-V$ colour curves are characterised by a nearly linear decline from red to blue colours, again over the period $\sim+30 \mathrm{~d}$ to $+90 \mathrm{~d}$.

Around $B$-band maximum and until the SNe Ia reach their respective maximum $B-V$ value, the colours vary in the sense that more-luminous SNe Ia exhibit bluer colours. This effect is likely tied to the ${ }^{56} \mathrm{Ni}$ production, the mixing of ${ }^{56} \mathrm{Ni}$, and hence the ionisation state of the ejecta (Kasen \& Woosley 2007). Quantifying the $B-V$ colours at maximum light $(+0 \mathrm{~d})$, we find values ranging from $\sim-0.07 \pm 0.01 \mathrm{mag}$ for $\mathrm{SN} 2006 \mathrm{dd}$, to between $\sim 0.01 \pm 0.01$ and $0.08 \pm 0.01 \mathrm{mag}$ for the transitional objects, to $\sim 0.73 \pm 0.01 \mathrm{mag}$ for the SN $1991 \mathrm{bg}$-like SN $2006 \mathrm{mr}$.

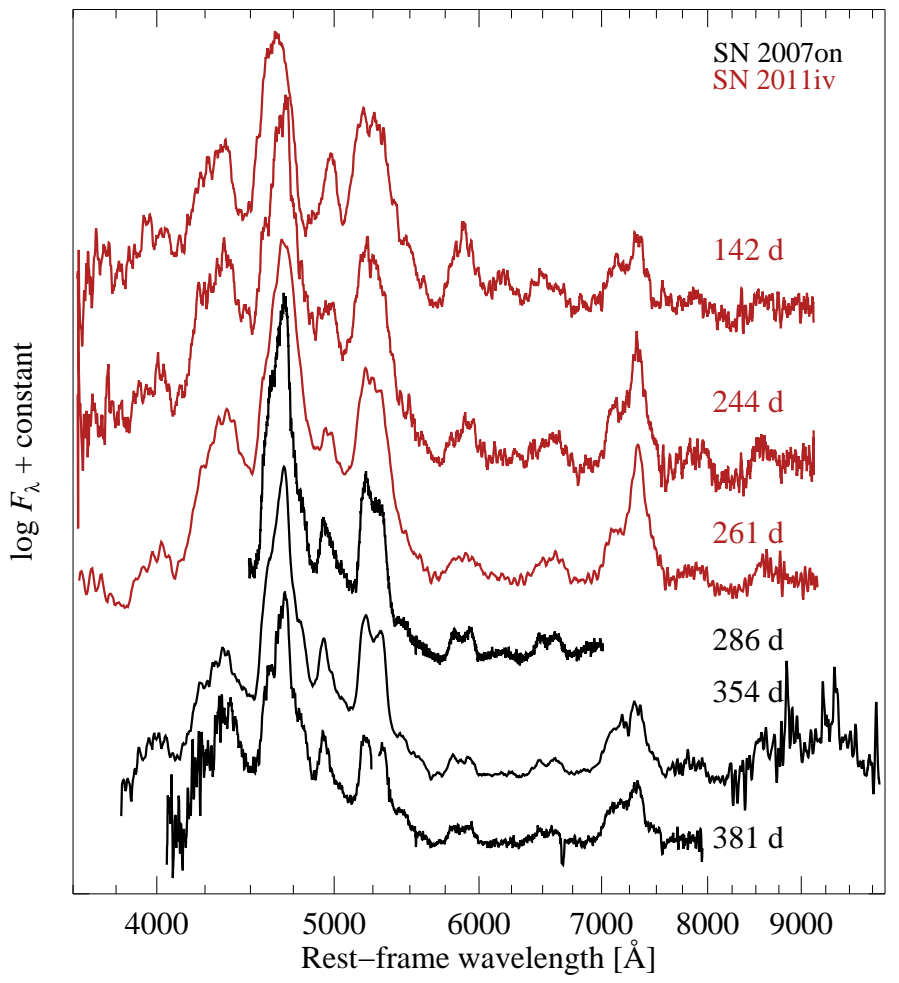

Fig. 6. Nebular-phase visual-wavelength spectra of SN 2007on (black) and SN 2011iv (red).

As the SNe Ia evolve beyond maximum light, the colour differences become even more discrepant (see Fig. 8). The subluminous SN $2006 \mathrm{mr}$ reaches its reddest $B-V$ colour value already at about $+10 \mathrm{~d}$, while the other objects peak later, around $+20 \mathrm{~d}$. Beginning around $+20 \mathrm{~d}$ and extending to beyond $+85 \mathrm{~d}$, the $B-V$ colours of SN 2007on are $\sim 0.12 \pm 0.01$ mag bluer than the colours of SN 2011iv, despite SN 2007on being fainter at $B$ band maximum. In Sect. 6.2 we speculate that this difference is due to intrinsic variations between the progenitor's central densities, and is not related to any effects of dust.

To further assess the colour evolution of SN 2007on and SN 2011iv, we examine their UV colours. We choose to use the Swift uvm 2 band because it gives the highest contrast to the optical bands. This is because it has a sharper cutoff at the longwavelength end of its passband as compared with the Swift uvw1 and $u v w 2$ bands. It is therefore more sensitive to UV effects (e.g. Brown et al. 2015). Displayed in Fig. 9 are the Swift uvm2-CSP $V$ colours (left panel), the CSP $u-B$ colours (middle panel), and the CSP $u-V$ colours (right panel) of SN 2007on and SN 2011 iv. At early epochs, where there are concurrent observations beginning around $-3 \mathrm{~d}$, the UV colours (uvm $2-V, u-V)$ of SN 2007on and SN 2011iv are quite similar, suggesting that the two objects have nearly the same conditions in their outer layers $\left(\sim 10^{-3}\right.$ $10^{-2} M_{\odot}$; see Appendix E). As the SNe Ia evolve, the UV colours of SN 2011 iv become redder at epochs past $+10 \mathrm{~d}$, resembling the $B-V$ colour evolution.

\subsection{Host-galaxy reddening}

According to the NASA/IPAC Extragalactic Database (NED), the Schlafly \& Finkbeiner (2011) recalibration of the Schlegel et al. (1998) dust maps suggests that the Galactic extinction component in the direction of NGC 1404 is $A_{V}^{\mathrm{MW}}$ $=0.031$ mag. Adopting a Fitzpatrick (1999) reddening law 


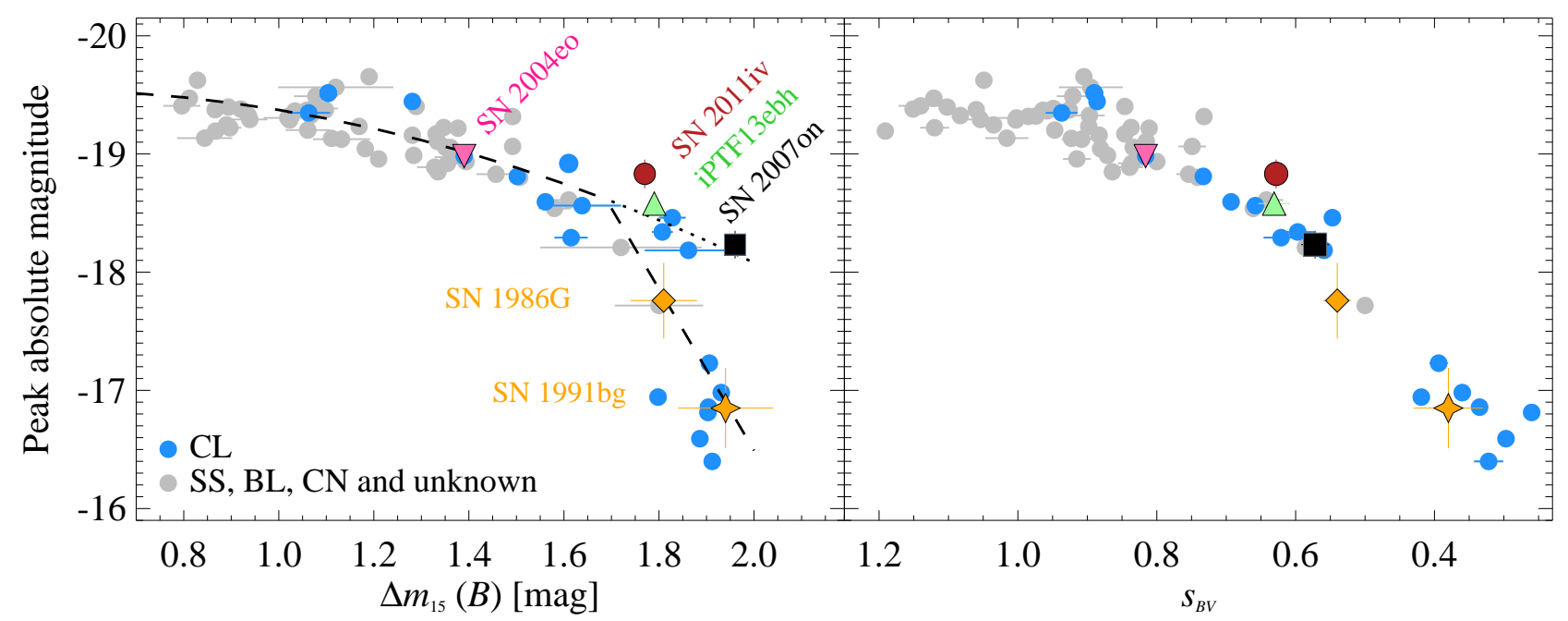

Fig. 7. Luminosity vs. decline-rate relation populated with a subset of CSP-I SNe Ia (Burns et al. 2014) and a few fast decliners from the literature. The relation is parameterised by $\Delta m_{15}$ (left) and $s_{B V}$ (right). The absolute $B$-band magnitudes are extinction corrected as described by Burns et al. (2014), and distances are computed using a Hubble constant $H_{0}=73 \mathrm{~km} \mathrm{~s}^{-1} \mathrm{Mpc}^{-1}$. A distance modulus of $\mu=31.27$ mag is used to place SN 2007on (black square) and SN 2011iv (red circle) on the luminosity scale. The blue circles correspond to SNe Ia classified as CL (cool) on the Branch et al. (2006) diagram, whereas the grey circles represent SNe Ia with either different (i.e. SS, CN, BL) or unknown Branch spectral subtype classifications. Additionally, included for comparison are the low-luminosity Type Ia SNe 1986G (yellow diamond) and 1991bg (yellow star), the transitional Type Ia iPTF13ebh (green triangle), and the normal Type Ia SN 2004eo (pink downward triangle). The dashed curves represent the parameterised luminosity vs. decline-rate relation for normal SNe Ia (Phillips et al. 1999) and for the subluminous SNe Ia (Taubenberger et al. 2008). The dotted curve represents an interpolation of the Phillips et al. (1999) relation for SNe Ia with $1.7<\Delta m_{15}(B)<2.0$ mag.

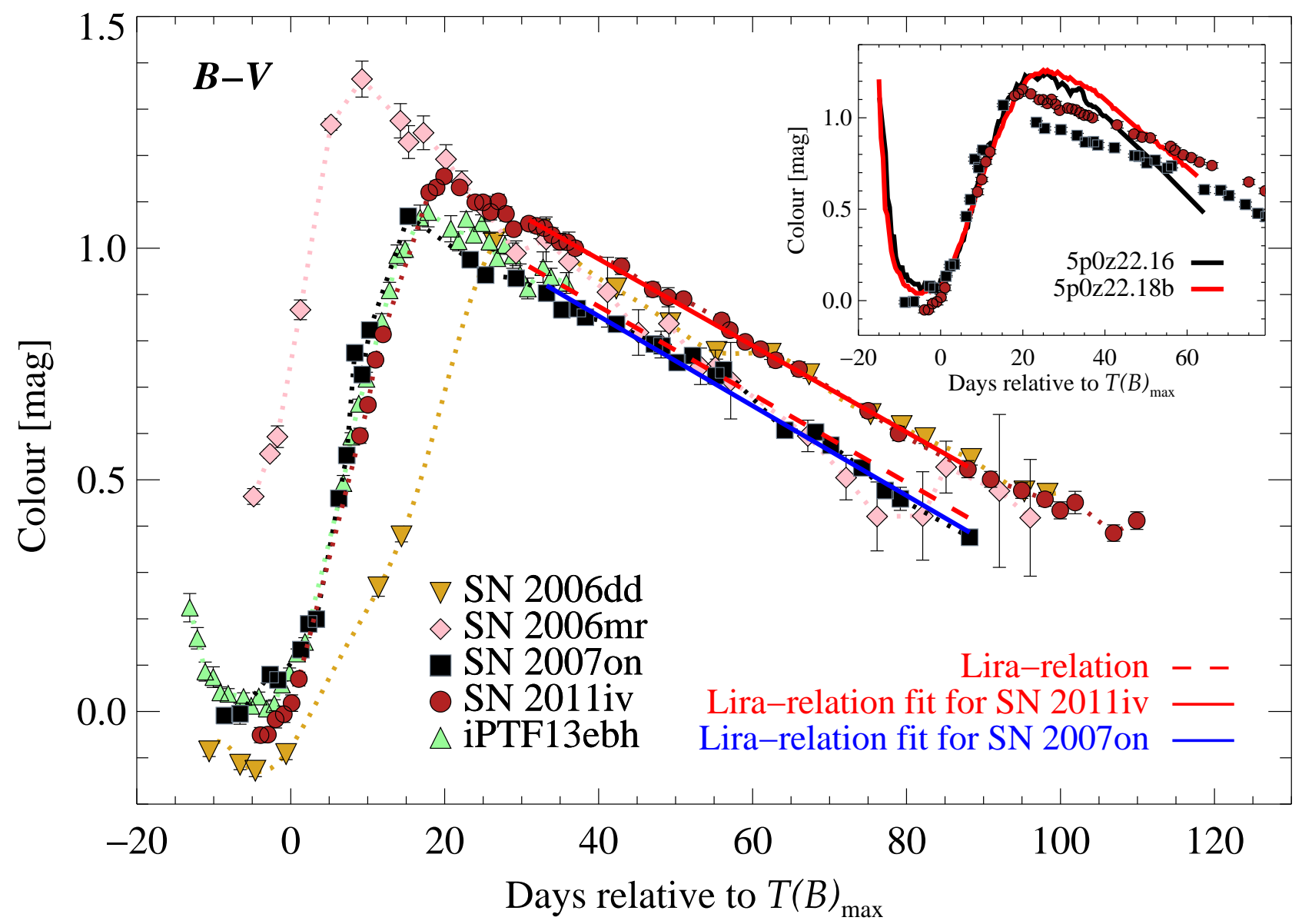

Fig. 8. Temporal evolution of the observed $B-V$ pseudo-colour. The filled red circles, black squares, light-green triangles, pink diamonds, and golden downward triangles represent the data of (respectively) SN 2011iv, SN 2007on, iPTF13ebh, SN 2006mr, and SN 2006dd. The data of the normal SN 2006dd and the subluminous SN 2006mr are from Stritzinger et al. (2010) and Contreras et al. (2010), respectively. All colours have been corrected for Galactic reddening. The Lira relation from Folatelli et al. (2010) is indicated as a red dashed line, and Lira-relation fits to the data are shown as red (SN 2011iv) and blue (SN 2007on) solid lines. The top-right inset contains the $B-V$ colour evolution of SN 2007on and SN 2011iv compared to their corresponding modelled $B-V$ colour evolution predicted by the best-fit DD models presented in Appendix D. 

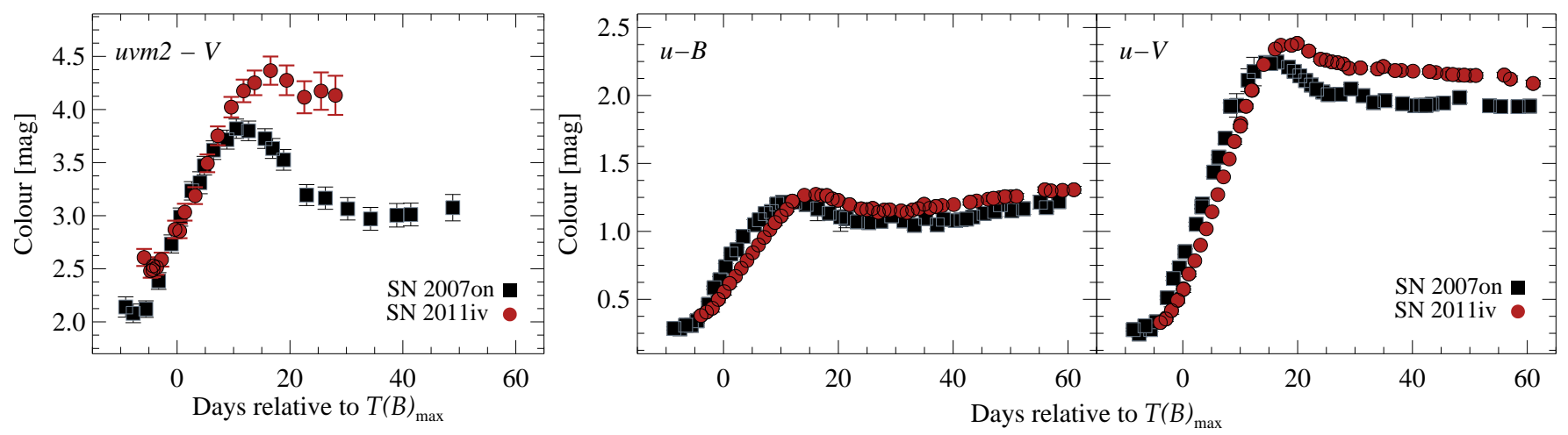

Fig. 9. Near-UV colours of SN 2007on (black squares) and SN 2011iv (red circles). Presented are the Swift uvm2 - CSP V colours (left panel), followed by CSP $u-B$ and $u-V$ colours in the middle and right panels, respectively.

characterised by $R_{V}=3.1$, this corresponds to a negligible $E(B-V)_{\mathrm{MW}}$ value of $0.01 \mathrm{mag}$.

Figure 10 (top panel) displays high-resolution visualwavelength spectra of SN 2007on and SN 2011iv zoomed in on the wavelength region where $\mathrm{NaI} \mathrm{D}$ absorption is expected. The +5 d spectrum of SN 2007on (Sternberg et al. 2014) was obtained with the Keck-I telescope (+ HIRES: High Resolution Echelle Spectrometer; Vogt et al. (1994)), and the $+55 \mathrm{~d}$ spectrum of SN 2011iv was obtained with the Magellan Clay telescope (+ MIKE: Magellan Inamori Kyocera Echelle). The spectra of both SNe Ia exhibit very weak Galactic NaI D absorption, which is consistent with the Schlegel et al. (1998) dust maps. On the other hand, no Na I D absorption components associated with the host galaxy are detected, suggesting minimal to no host-galaxy reddening. An examination of the UV spectra of SN 2011iv (bottom panel of Fig. 10) reveals several narrow interstellar absorption lines including Fe II $\lambda \lambda 2344,2374,2382$, 2586, and 2600, and Mg II $\lambda \lambda 2796,2803$. Close inspection of these absorption features show that they are not associated with material located at the redshift of the host galaxy, but rather originate from material within the Milky Way located along the line of sight to NGC 1404.

Estimates of host reddening for SN 2007on and SN 2011iv are made by comparing the observed peak colours to the intrinsic peak colours as defined by a large sample of minimally reddened SNe Ia (Burns et al. 2014). Using SNooPy's "EBV_method2" (see, e.g. Stritzinger et al. 2010, Eq. (6)), we compute template light-curve fits that imply host-galaxy colour excess values of $E(B-V)_{\text {host }}^{07 \text { on }}=-0.06 \pm 0.01$ (random) \pm 0.06 (systematic) mag and $E(B-V)_{\text {host }}^{11 \text { iv }}=-0.02 \pm 0.01$ (random) \pm 0.06 (systematic) mag, both consistent with minimal to no host reddening.

Host-reddening estimates may also be obtained using the Lira relation (Lira 1996). The Lira relation is based on the empirical trend that the $B-V$ colours of normal, minimally reddened SNe Ia evolve uniformly from $+30 \mathrm{~d}$ to $+90 \mathrm{~d}$ with a linear slope, and it has been found to also apply to a sample of fast-declining SNe Ia (e.g. Taubenberger et al. 2008). However, Burns et al. (2014) suggest a parameterisation of the Lira law using the colour-stretch parameter, $s_{B V}$, to account for a potentially steeper $B-V$ slope such as exhibited by SN $1991 \mathrm{bg}-$ like $\mathrm{SNe}$ Ia. Here we adopt the Lira-relation as parameterised by Folatelli et al. (2010), which is shown in Fig. 8. Comparing the offset of the observed $B-V$ colour evolution to that of the Lira relation between $+30 \mathrm{~d}$ and $+90 \mathrm{~d}$, we obtain host-galaxy colourexcess values of $E(B-V)_{\text {Lira }}^{07 \text { on }}=-0.025 \pm 0.01$ (random) \pm 0.04 (systematic) mag and $E(B-V)_{\text {Lira }}^{11 \text { iv }}=0.1 \pm 0.01$ (random) \pm 0.04
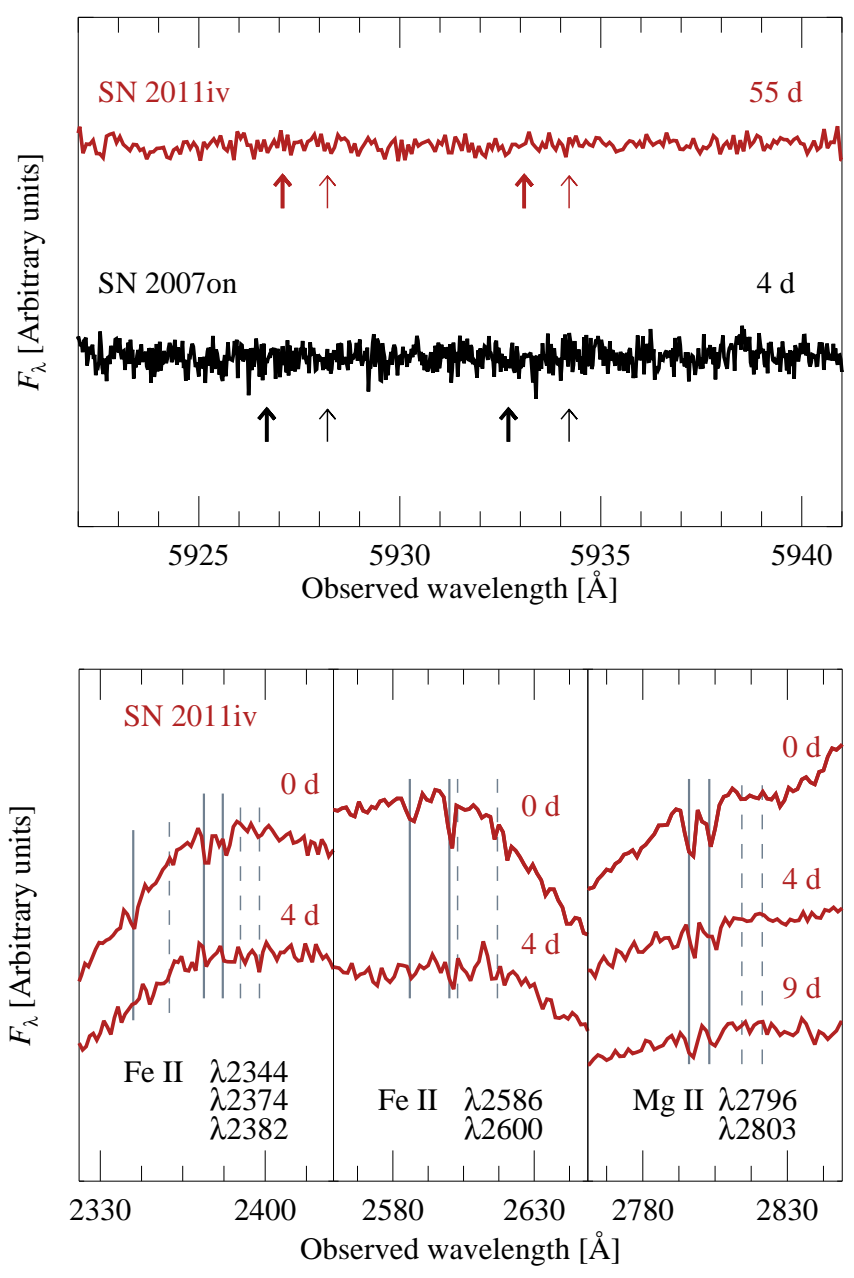

Fig. 10. Host and interstellar absorption. (top) High-resolution visualwavelength spectra of SN 2011iv (top) and SN 2007on (bottom) (Sternberg et al. 2014), zoomed in at the expected location of the hostgalaxy Na I D absorption. The thin arrows indicate the positions of the $\mathrm{Na}$ I D lines at the heliocentric velocity of NGC 1404, while the thick arrows indicate the expected positions based on the rotation curve of the host (Graham et al. 1998). In both cases, no Na I D lines are discernible, suggesting minimal to no host-galaxy reddening. (bottom) Interstellar Fe and Mg absorption features in UV spectra of SN 2011iv. The spectra around maximum light of SN 2011 iv exhibit narrow absorption features of Fe II $\lambda 2344$, Fe II $\lambda 2374$, and Fe II $\lambda 2382$ (left panel), Fe II $\lambda \lambda 2586$, 2600 (middle panel), and Mg II $\lambda \lambda 2796,2803$ (right panel) at the position of the Milky Way (solid grey lines). The dashed grey lines indicate the expected position of the absorption features, if they originate from the host galaxy. 

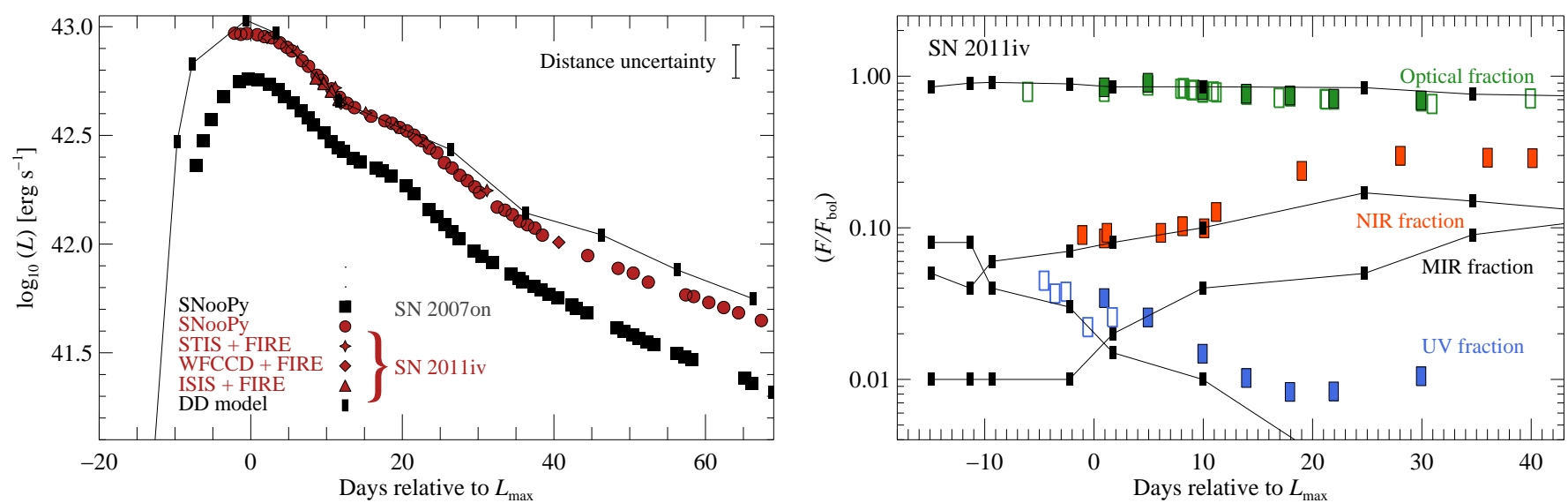

Fig. 11. Left: blometric light curves of SN 2007on (black) and SN 2011 iv (red) constructed from observations constraining the flux extending from uvm 2 to the $H$ band ( 2250-18 $800 \AA$ A; UVOIR). Complementing the majority of SN 2011iv measurements are a handful of additional bolometric points covering the wavelength range extending from the UV (1620 ̊) to $K_{\mathrm{s}}(24900 \AA)$. The error bar on the bolometric luminosity is presented in the top right of the plot, and assumes an error in the adopted distance modulus of \pm 0.2 mag. Also shown is the bolometric light curve (line) of model 5p0z22.18b presented in Sect. 6.2. Right: UV (blue), optical (green), and NIR (red) flux fractions of the total bolometric light curve of SN 2011 iv computed from observed spectroscopy. The open green symbols illustrate reduced optical flux fractions from $3480 \AA$ to $8630 \AA$, while the solid green symbols represent the full range from the atmospheric cutoff at $3050 \AA$ to $8630 \AA$. The UV fraction extends from either $1620 \AA$ (STIS, solid blue symbols) or $1900 \AA$ (UVOT, open blue symbols) to $3050 \AA$, and the NIR fraction extends from $9000 \AA$ to $24900 \AA$. Shown as connected filled squares is the fraction of flux distributed among the different wavelength regimes for model 5p0z22.18b. This includes the predicted MIR fraction (24900 ̊ to $1000000 \AA$ ), which drives the difference between the observed and modelled UVOIR light curves beginning around a month past maximum brightness.

(systematic) mag. In what follows, SN 2007on and SN 2011iv are assumed to have zero host-galaxy extinction.

\subsection{Bolometric light curves and ${ }^{56} \mathrm{Ni}$ estimates}

The exquisite observational datasets of SN 2007on and SN 2011iv enable us to construct comprehensive bolometric (UVOIR) light curves with the use of the bolometric function contained within SNooPy. The function has several different ways to construct the bolometric light curve. In our case we adopted the so-called SED (spectral energy distribution) method. In this method the $u B g V r i Y J H$-band light curves were first fit with Gaussian process spline functions. The resultant spline functions were then used to estimate the colours of the SN on the same phases of the Hsiao et al. (2007) spectral templates 5 . The spectral templates were then multiplied by an appropriate bspline function, which ensured their synthetic colours match the observed colours. Next, flux bluewards of the atmospheric cutoff ( $\approx 3050 \AA$ ) of the spectral templates was estimated by interpolating to the $u v m 2$ flux point. The total flux was then obtained by integrating from the effective wavelength of the $u v m 2$ passband $(\approx 2250 \AA)$ to the red edge of the $H$ band $(\approx 18800 \AA)$. Finally the integrated flux was de-reddened and placed on the absolute luminosity scale using the adopted distance discussed in Sect. 1.

Figure 11 (left panel) displays the definitive bolometric light curves of SN 2007on and SN 2011iv and the corresponding luminosity values are listed in Tables F.8 and F.9, respectively. The figure also shows an indication of the uncertainty in the bolometric luminosity adopting an uncertainty in the adopted distance modulus of $\pm 0.20 \mathrm{mag}$. Additionally, for SN 2011iv there are a handful of spectrophotometric bolometric points constructed by integrating the SN Ia flux from combined UV, optical, and NIR spectra; these are reported in Table F.10. Given that

\footnotetext{
5 SNooPy has an updated set of spectral templates extending from optical to NIR wavelengths.
}

the NIR spectra include the additional wavelength region corresponding to the $K_{\mathrm{s}}$ band, the spectrophotometric bolometric points tend to have an ever-increasing amount of luminosity relative to maximum as compared to the bolometric points obtained from the photometry. The time dependence of the difference between the photometric vs. spectrophotometric bolometric points reflects the increasing fraction of flux emitted at red wavelengths as the SED evolves. Also shown in the left panel of Fig. 11 is the bolometric light curve corresponding to the best-fit DD model of SN 2011 iv presented below (see Sect. 6.2 and Appendix D).

The bolometric light curves indicate that SN 2011iv is more luminous than SN 2007on at all epochs. Quantitatively, the peak luminosity obtained for SN 2007on is $L_{\mathrm{bol}}^{\max }=$ $(5.05 \pm 0.8) \times 10^{42} \mathrm{erg} \mathrm{s}^{-1}$, and for SN 2011iv it is $L_{\mathrm{bol}}^{\max }=$ $(9.03 \pm 0.9) \times 10^{42} \mathrm{erg} \mathrm{s}^{-1}$. The peak luminosities are used to estimate the ejected ${ }^{56} \mathrm{Ni}$ abundance through application of Arnett's rule (Arnett 1982). Following the method described by Stritzinger \& Leibundgut (2005), and adopting the same rise time as employed for modelling the maximum-light optical spectra (Sect. 5.2, Fig. 15), ${ }^{56} \mathrm{Ni}$ yields of $0.24 \pm 0.05 M_{\odot}$ and $0.43 \pm 0.06 M_{\odot}$ are computed for SN 2007on and SN 2011iv, respectively.

Given the broad wavelength coverage obtained for SN 2011iv, we show in Fig. 11 (right panel) the fraction of the total bolometric flux in the UV, optical, and NIR regimes estimated using observed spectroscopy Clearly, the contribution from optical wavelengths (3050-8630 ̊) dominates the total flux at all phases. At maximum brightness, the fraction of flux blueward of the atmospheric cutoff is less than $10 \%$, while in the NIR extending from $9000 \AA$ out to $24900 \AA$, it accounts for about $10 \%$. By a month past maximum the UV fraction drops to less than $1 \%$, while the NIR contribution increases to about $30 \%$. Also shown is the fraction of flux distributed among the different wavelength regimes for the best-fit DD model of SN 2011iv, including the fraction of flux in the mid-IR (MIR). 


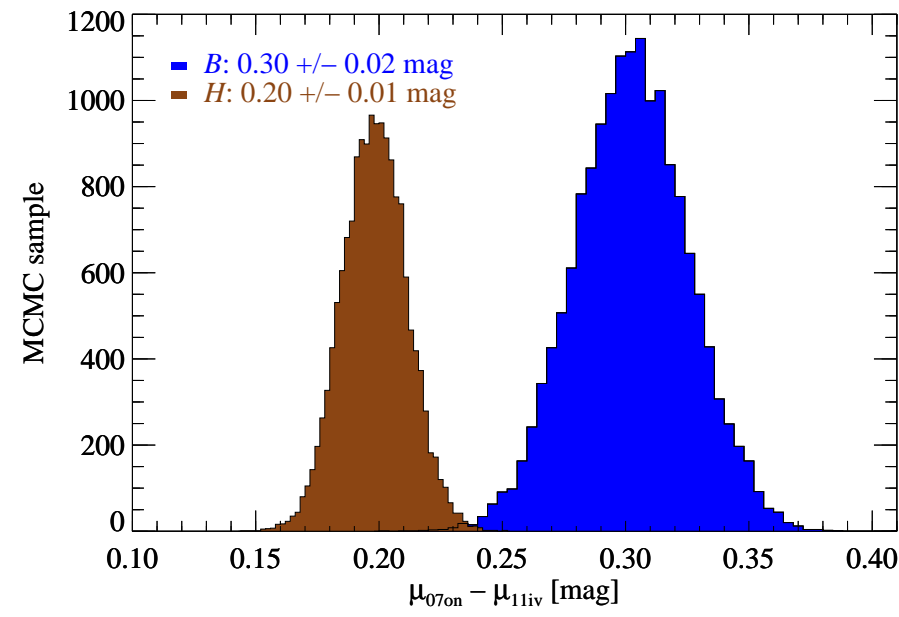

Fig. 12. Probability distribution of the differences in dereddened distance computed for SN 2007on and SN 2011iv from their peak $B$ - and $H$-band magnitudes, after having applied corrections for the luminosity vs. colour and luminosity vs. decline-rate relations. The $H$-band distribution is narrower because it is less sensitive to errors in the extinction correction.

\section{Type la supernova distance to NGC 1404}

Equipped with the optical and NIR light curves of SN 2007on and SN 2011iv, we can use them to independently ascertain the distance to their host galaxy, NGC 1404. We focus our efforts on determining the relative difference between the distances inferred from SN 2007on and SN 2011iv, rather than on their absolute distances.

Assuming SN 2007on and SN 2011iv are indeed located in NGC 1404, two factors are key for explaining the difference in their observed flux: extinction owing to dust and the luminosity vs. decline-rate relation (Phillips et al. 1999). To investigate this, we construct a Markov chain Monte Carlo (MCMC) simulation that models the distance to each object as a function of its observed magnitude. For details of the procedure, see Burns et al. (2011). To compute the host extinction, we use the results from Burns et al. (2014) assuming the Gaussian mixture model as a prior for $R_{V}$. Similar to what was discussed previously (Sect. 3.3), here we also found that both objects only suffer minimal to no host reddening. However, it is important to include the uncertainties and their covariances in the analysis. From the resulting MCMC chains, we compute the statistics on the "difference" between the distances of SN 2007on and SN 2011iv for each observed passband.

Interestingly, the distances derived from the optical bands are more discrepant between the two $\mathrm{SNe}$ than the distances derived using the NIR bands. This is demonstrated in Fig. 12, which shows the probability distribution of the differences in distance moduli computed for the $B$ - and $H$-band peak luminosities of our two $\mathrm{SNe}$ after applying the luminosity vs. colour and luminosity vs. decline-rate corrections. The probability distributions of the difference in distance moduli is narrower in the $H$ band because the corrected NIR luminosities are less sensitive to errors in the extinction correction. Quantitatively, the $B$-band distance modulus of SN 2007on is $\left(\mu_{0}-5 \log _{10} \cdot h_{73}\right)=31.37 \pm 0.024$ (random) mag, where $h_{73}=H_{0} /\left(73 \mathrm{~km} \mathrm{~s}^{-1} \mathrm{Mpc}^{-1}\right)$, and the $H$-band distance modulus is $\left(\mu_{0}-5 \log _{10} \cdot h_{73}\right)=31.31 \pm 0.030$ (random) mag. For SN 2011iv, on the other hand, the $B$-band distance modulus is $\left(\mu_{0}-5 \log _{10} \cdot h_{73}\right)=31.07 \pm 0.021 \mathrm{mag}$ and the $H$-band distance modulus is $\left(\mu_{0}-5 \log _{10} \cdot h_{73}\right)=31.11 \pm 0.023 \mathrm{mag}$.
These values yield differences of $\Delta \mu=0.30 \pm 0.02$ mag and $\Delta \mu=0.20 \pm 0.01 \mathrm{mag}$ in the $B$ band and $H$ band, respectively (see Fig. 12). These comparisons correspond to $\sim 14 \%$ and $\sim 9 \%$ differences in distance.

\section{Spectral analysis}

\subsection{HST ultraviolet spectroscopy}

\subsubsection{Observations and spectral comparison}

The UV spectrum of a SN Ia is shaped by a complex set of processes, including significant line-blanketing driven by Fe-group elements at various ionisation states (see, e.g. Mazzali 2000). The UV spectral region therefore offers an opportunity to study the ${ }^{56} \mathrm{Ni}$ and $\mathrm{Fe}$-group element content located in the outer layers of the expanding ejecta (e.g. Hoeflich et al. 1998; Lentz et al. 2000; Timmes et al. 2003; Sauer et al. 2008; Walker et al. 2012).

Given the limited observational coverage to date of the UV portion of SNe Ia, we have undertaken a detailed examination of our UV spectroscopic time-series (Sect. 2.2). From Fig. 3, it is evident that SN 2011iv is rich in prominent P-Cygni features, including those located at $2300 \AA, 2500 \AA, 2650 \AA$, $3000 \AA$, and $3200 \AA$, which are typically caused by $\mathrm{Mg}$ II, Fe II, and other Fe-group elements. The early-epoch $(+0 \mathrm{~d}$ to +9 d) spectra also exhibit a conspicuous feature around $1900 \AA$. A similar feature typically attributed to Fe II or Co II is also present in the normal Type Ia SN 1992A (Kirshner et al. 1993) and SN 2011fe (Mazzali et al. 2014), as well as in the Type IIP SN 1999em (Bufano et al. 2009) and the Type IIb SN 2001ig (Ben-Ami et al. 2015).

Contained within the inset of Fig. 3 is a comparison between the maximum-light spectrum of SN 2011iv to similarepoch spectra of the normal Type Ia SN 2011fe (Mazzali et al. 2014) and SN 2013dy (Pan et al. 2015). SN 2011iv clearly shows notable differences in the UV spectral range. As demonstrated in the inset of the left panel, the flux level in the range 1600$2600 \AA$ differs marginally between the depicted SNe Ia. However, the inset in the right panel reveals a significantly lower flux level in SN 2011iv at 2600-3600 $\AA$ as compared to the other objects. Furthermore, significant differences in line strengths, shapes, and locations are apparent for the most prominent features.

SN 2011fe and SN 2013dy are chosen for comparison because they are both normal objects with high-quality data obtained at similar wavelengths and epochs. Furthermore, a similar comparison as shown in the insets of Fig. 3 has been discussed elsewhere (Foley et al. 2012b, 2016; Pan et al. 2015). The origin of the notable flux-level differences exhibited by SNe Ia in the UV is a matter of open debate. Detailed modelling of SN Ia spectra points to a rather complex interplay between various physical parameters. This includes ejecta stratification (mixing), metallicity, modification of density structures due to pulsations (Gerardy et al. 2004), spectral line formation and ionisation states (e.g. Hoeflich et al. 1998; Lentz et al. 2000; Mazzali et al. 2014; Sauer et al. 2008), as well as geometric and viewing-angle effects that may alter the UV flux level (e.g. Kromer \& Sim 2009). In the remainder of this section, we quantify the similarities and differences between the UV properties of SN 2011iv and other normal SNe Ia.

\subsubsection{UV pseudo-equivalent width}

To quantify the UV spectral properties of the two prominent absorption features indicated in Fig. 3 (left panel), measurements 
Table 2. UV feature limits for the pseudo-equivalent width.

\begin{tabular}{cccc}
\hline \hline Feature & $\begin{array}{c}\text { Position/ } \\
\text { Wavelength } \\
{[\AA]}\end{array}$ & $\begin{array}{c}\text { Blueward } \\
\text { limit range } \\
{[\AA]}\end{array}$ & $\begin{array}{c}\text { Redward } \\
\text { limit range } \\
{[\AA]}\end{array}$ \\
\hline$p W 01$ & Mg II 2800 & $2580-2620$ & $2700-2750$ \\
$p W 02$ & $\sim 2500$ & $2380-2420$ & $2580-2620$ \\
\hline
\end{tabular}

are made of their pseudo-equivalent width ( $\mathrm{pEW}$ ), which is a common method to quantify spectral properties of $\mathrm{SNe}$ Ia at optical wavelengths (e.g. Folatelli 2004; Garavini et al. 2007; Folatelli et al. 2013). Parameters that define the two UV pEW indicators considered here were obtained following the prescription of Garavini et al. (2007) and are summarised in Table 2. In short, the two pEW indicators are defined from the blue to the red maximum of an absorption trough.

Based on this definition, Fig. 13 shows the resulting temporal evolution of the derived UV pEWs for SN 2011iv, as well as for the comparison SNe (SN 1992A, SN 2011fe, and SN 2013dy), chosen as they are representatives of normal SNe Ia with excellent UV data. Interestingly, the pEW values of SN 2011iv resemble those of SN 2011fe, while the pEW values of SN 1992A evolve similar to those of SN 2013dy. The pEW values of pW02 appear to increase until around $+10 \mathrm{~d}$ past maximum light, whereafter the $\mathrm{pEW}$ steadily declines in a similar fashion for the considered SNe Ia.

\subsubsection{The $3000 \AA$ feature}

Features at longer wavelength (i.e. $\gtrsim 2800 \AA$ ) are formed well within the SN ejecta and are products of nuclear burning (e.g. Branch \& Venkatakrishna 1986; Kirshner et al. 1993; Sauer et al. 2008). Conspicuous features in this region are those around $3000 \AA$ and $3250 \AA$, which are blends of Fe II or Co II (e.g. Branch \& Venkatakrishna 1986). Comparing the $3000 \AA$ absorption feature of the maximum-light spectrum of SN 2011iv (Fig. 3) with that of other SNe Ia reveals noticeable differences in its shape. SN 2011iv and SN 2011fe exhibit a broad "Wshaped" absorption trough, while in the case of SN 2013dy this feature is "V-shaped". A V-shaped feature is also present in SN 1992A (Kirshner et al. 1993). We measured the pEW of the entire $3000 \AA$ feature for epochs around maximum light in a similar fashion as for the other UV features and find values of about $40 \AA$, in agreement with measurements in the literature (e.g. Foley et al. 2008).

\subsubsection{No evidence of SN la ejecta and circumstellar interaction}

UV observations have been used in the past to provide constraints on interaction between the expanding SN Ia ejecta and circumstellar material (CSM). In Appendix E we discuss our efforts to search for evidence of interaction between the ejecta of SN 2011iv and any possibly CSM. In short, our efforts yield no evidence of interaction in the case of SN 2011iv.

\subsection{Optical spectral comparison of early epochs and spectral synthesis}

Unlike the UV spectral range that is distinguished by significant line blending of various $\mathrm{Fe}$-group elements in the outermost layers, the visual-wavelength range is characterised by a variety of

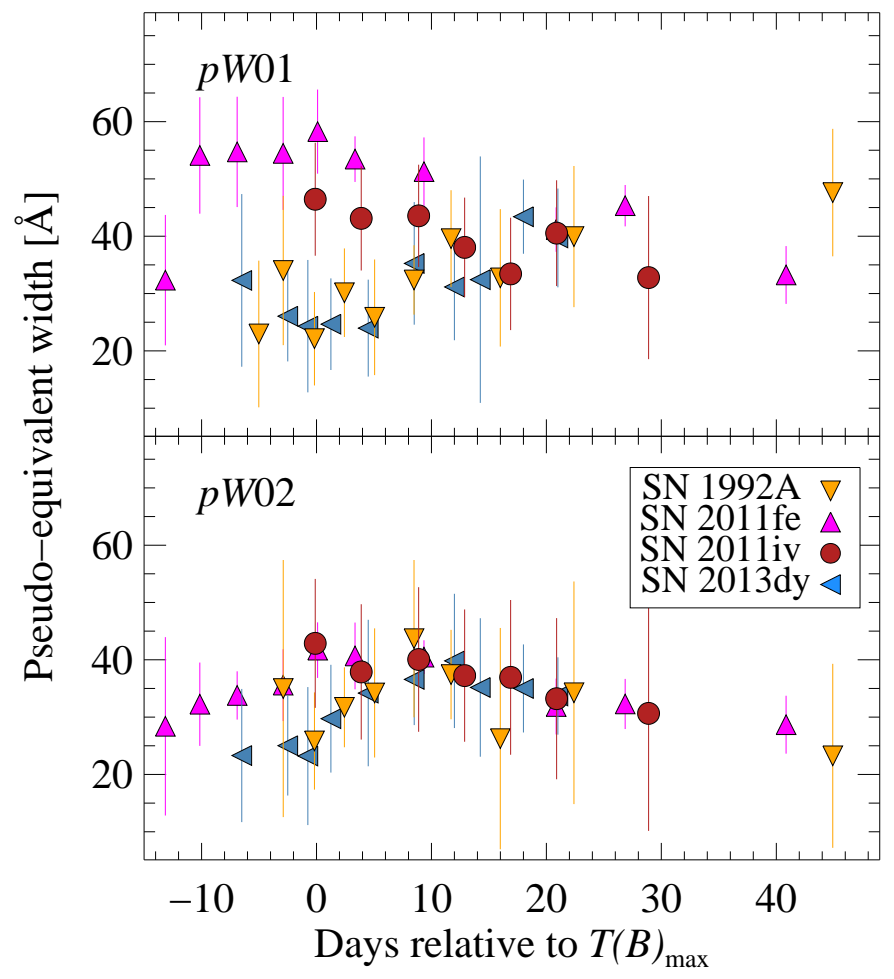

Fig. 13. The $\mathrm{pEW}$ of the prominent $U V$ spectral absorption features designated pW01 and pW02. The pEW measurements are presented for SN 1992A (Kirshner et al. 1993, yellow downward triangles), SN 2011fe (Mazzali et al. 2014, pink upward triangles), SN 2011iv (red circles), and SN 2013dy (Pan et al. 2015, blue left-sided triangles).

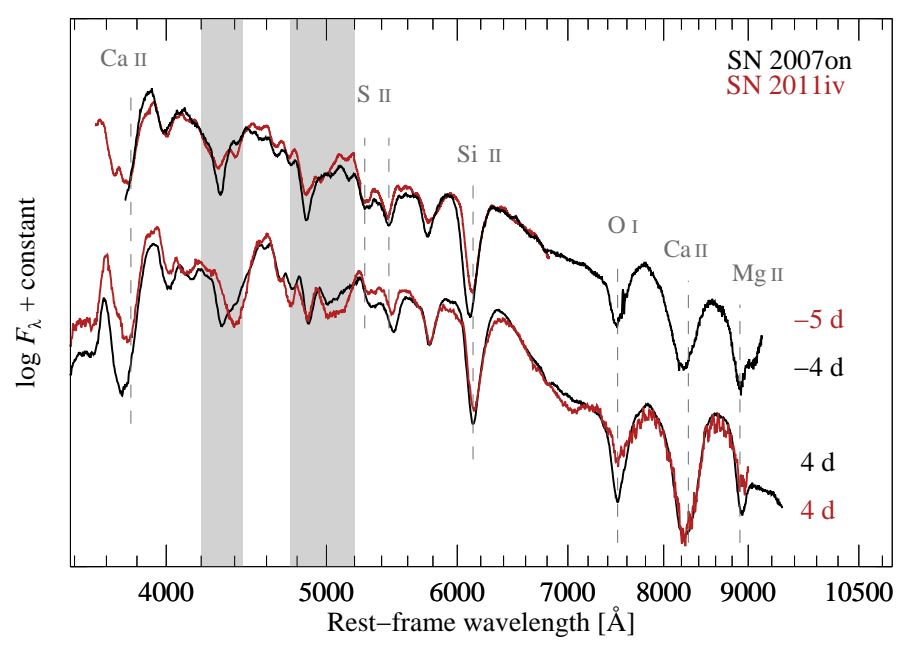

Fig. 14. Comparison of visual-wavelength spectra of SN 2007on and SN 2011 iv taken around maximum light. As described in Sect. 5.2, the grey vertical bands highlight prominent features around 4200-4400 and 4800-5200 $\AA$, where the two objects show clear disagreement.

broad P-Cygni profiles from single elements (see, e.g. Fig. 4). The study of these features and their temporal evolution can provide clues regarding SN Ia progenitors and an avenue to constrain the explosion physics.

Figure 14 presents a comparison between visual-wavelength spectra of SN 2007on and SN 2011iv taken around $-5 \mathrm{~d}$ and $+4 \mathrm{~d}$, along with identifications for all of the prominent spectral features. Overall, the spectra are similar, and all of the main ions that characterise a SN Ia are present. However, as highlighted 
by the shaded regions in Fig. 14, notable spectral differences are evident at 4300-4500 $⿱$ and 4700-5200 $\AA$. Additionally, there are some small differences in the blueshifts of the absorption minimum of the Si II $\lambda 6355$ line.

To facilitate the identification of the various spectral features observed in the early epochs of SN 2007on and SN 2011iv, and in particular, in the first two shaded regions of Fig. 14, we turn to spectral synthesis modelling using well-established techniques applied to the study of numerous SNe Ia including the normal SN 2011fe (Mazzali et al. 2014) and SN 2014J (Ashall et al. 2014). Synthetic spectra are computed based on the W7 density profile (Nomoto et al. 1984), allowing for consistency between line identification with other models. We note that the majority of SN Ia explosion models produce similar-looking density profiles in the regions where the maximum-light spectrum is formed; the main differences are in the outermost velocities. Therefore, the results presented below are independent of whether we use a W7 density profile or that associated with our best-fit DD explosion models (see Sect. 6.2).

Our spectral synthesis calculates the radiation field above a blackbody photosphere, with element abundances, luminosity, and photospheric velocity varied to produce a best-fit spectrum (Mazzali 2000). The code uses the density profile of the "fast deflagration" single-degenerate W7 model (Nomoto et al. 1984; Iwamoto et al. 1999).

Figure 15 shows the synthetic spectral models computed for the $-1.0 \mathrm{~d}$ spectrum of SN 2007on and the spectrum of SN 2011iv taken at maximum light. The model parameters for the premaximum spectrum of SN 2007on imply a photospheric velocity $v_{\mathrm{ph}}=9500 \mathrm{~km} \mathrm{~s}^{-1}$, a photospheric blackbody temperature $T_{\mathrm{ph}}=10600 \mathrm{~K}$, a bolometric luminosity $L_{\mathrm{bol}}=$ $4.80 \times 10^{42} \mathrm{erg} \mathrm{s}^{-1}$, and a rise time to maximum $t_{\text {rise }}=17.4 \mathrm{~d}$. In the case of the synthetic spectrum of SN 2011iv, the model parameters are $v_{\mathrm{ph}}=9500 \mathrm{~km} \mathrm{~s}^{-1}, T_{\mathrm{ph}}=10700 \mathrm{~K}, L_{\mathrm{bol}}=$ $7.03 \times 10^{42} \mathrm{erg} \mathrm{s}^{-1}$, and $t_{\text {rise }}=17.9 \mathrm{~d}$.

The models consist of spectral features attributed to many of the same ions found in the spectra of normal SNe Ia (see Fig. 15), and some of the features are produced by the blending of several ions. Prominent spectral features are attributed to various ions of intermediate-mass elements including Ca II H\&K, Si II $\lambda \lambda 3856,4130,5972,6355$, S II $\lambda \lambda 5449,5623$, and the Ca II NIR triplet. The prevalent feature seen around $7500 \AA$ is dominated by a blend of $\mathrm{O}$ I $\lambda 7773$, which is a characteristic of subluminous SN 1991bg-like SNe Ia (e.g. Garnavich et al. 2004; Taubenberger et al. 2008; Ashall et al. 2016b).

The spectral feature located at 4300-4500 A in SN 2011iv is broader than in SN 2007on (see first shaded region in Fig. 14). The synthetic spectra of SN 2011 iv have a more significant contribution of Si III $\lambda 4553$ and Fe III $\lambda 4420$, whereas in the case of SN 2007on this region is dominated by Mg II $\lambda 4481$.

Moving to longer wavelengths, our models indicate that the prominent feature in SN 2011 iv at 4700-5000 $\AA$ is formed from a blend of various Si II and Fe III lines, with the strongest contributions from Si II $\lambda 5055$ and Fe III $\lambda 5156$, and it contains only a small contribution of Fe II and S II. In the case of SN 2007on, this region in the synthetic spectrum is formed by $\mathrm{Si}$ II $\lambda 5055$, with some contribution from S II $\lambda 5032$ and Fe II $\lambda$ 15169. The differences between the spectra of SN 2007on and SN 2011iv are attributed to the latter being intrinsically hotter, which translates into it having ejecta characterised by a higher ionisation state. A full detailed spectral analysis using the abundance tomography technique will be presented in an forthcoming paper (Ashall et al. 2018).
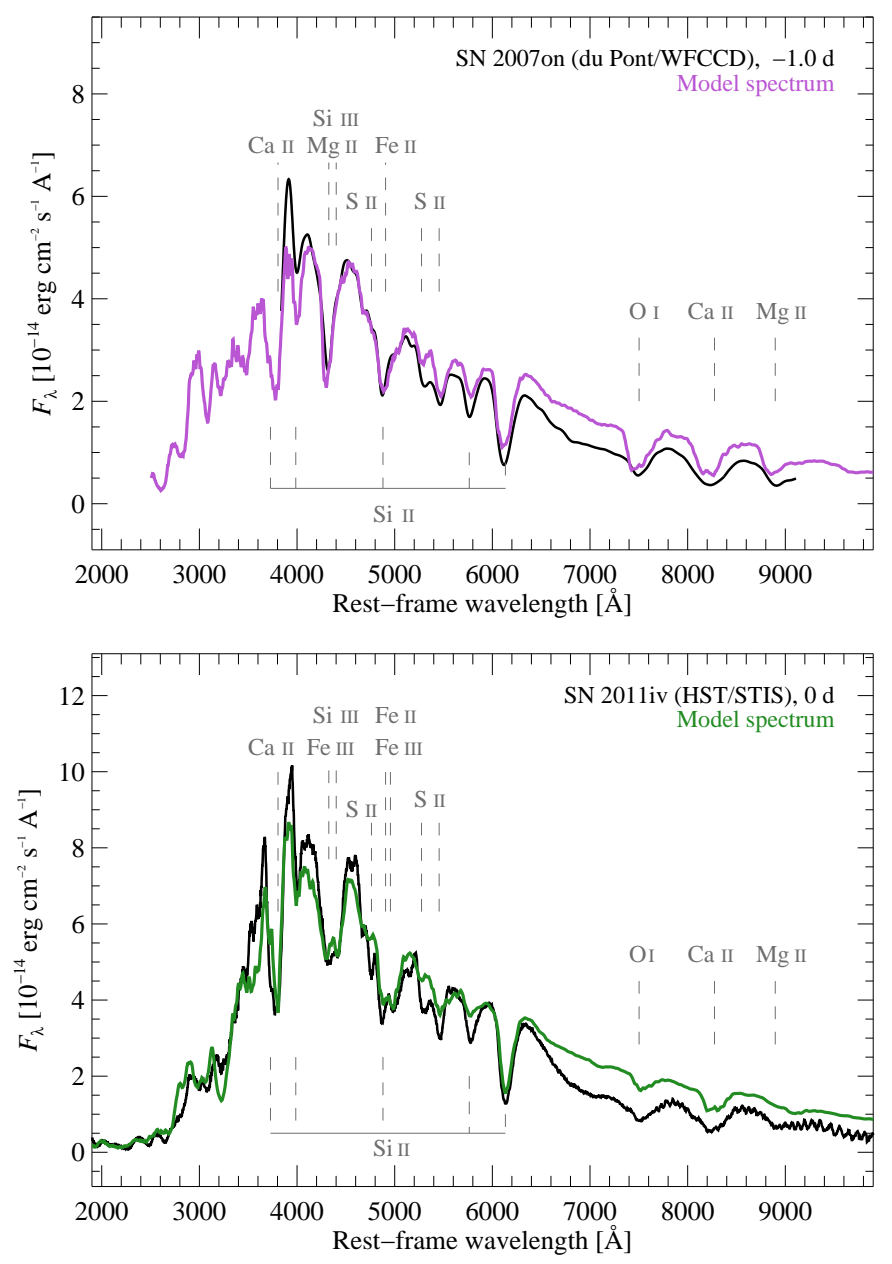

Fig. 15. Near-maximum-light visual-wavelength spectra (black) of SN 2007on (top) and SN 2011iv (bottom) compared to our best-fit modelled spectrum.

\subsection{NIR Spectroscopy}

At early epochs, the NIR spectra (see Fig. 5) of SN 2011iv are rather smooth, exhibiting only a handful of features, including the Mg II $\lambda 9218$ line and an absorption dip at $\sim 1.6 \mu \mathrm{m}$, which is associated with the $H$-band break. As the spectrum evolves with time, a multitude of features attributed to Fe-group elements (such as Fe II, Co II, and Ni II) emerge and dominate the spectrum, particularly at the wavelength regions corresponding to the $H$ and $K_{\mathrm{s}}$ bands. The prominent feature at $\sim 9000 \AA$ may be attributed to a blend of Mg II $\lambda \lambda 9218,9244$, as was identified in the transitional iPTF13ebh (Hsiao et al. 2015). At epochs beyond $+10 \mathrm{~d}$, the latter feature becomes broader and even more prominent, together with a feature located around $\sim 9800 \AA$. These absorption troughs are likely the result of line blanketing of a large number of $\mathrm{Mg}$ II, Ca II, Co II, and Fe II lines (Hoeflich et al. 2002).

Hsiao et al. (2015) provided a detailed study of the $H$-band break observed in SN 2011iv, which is located right between the two major telluric regions in the NIR. In summary, the profile of the $H$-band break of SN 2011 iv evolves differently than, say, that of iPTF13ebh, despite both objects being very similar otherwise (see Hsiao et al. 2015). The feature is weaker in strength compared to normal SNe Ia (see Hsiao et al. 2015, Fig. 15), though the peak of the $H$-band break ratio of SN 2011iv appears to fit 
into the correlation with the light-curve decline rate, $\Delta m_{15}$, and the colour-stretch parameter, $s_{B V}$ (Hsiao et al. 2013).

The velocities of the NIR Mg II 19218 (Sect. B.2, Fig. B.2) feature appear to decrease consistently in SN 2011iv and are similar to those observed in the fast-declining SN 1991bg and iPTF13ebh. High velocities at early phases also characterise the $\mathrm{Mg}$ II $\lambda 1.0972 \mu \mathrm{m}$ line of iPTF13ebh and SN 2011 iv (Hsiao et al. 2015, Fig. 16).

Displayed in Fig. 16 are combined visual-wavelength and NIR spectra of SN 2007on and SN 2011 iv taken around a month past maximum brightness, with line identifications provided for the most prevalent features. The spectra have been calibrated through multiplication by a function that ensures their synthetic broad-band magnitudes match the observed broad-band magnitudes inferred from the interpolated optical and NIR photometry on $+29 \mathrm{~d}$. The comparison shows that the objects are overall quite similar. The spectral features of both objects also remain similar at all wavelengths, including the Co II features located between $2.0 \mu \mathrm{m}$ and $2.4 \mu \mathrm{m}$.

\subsection{Nebular spectroscopy}

The late-phase visual-wavelength spectra of SN 2007on and SN 2011iv provide an opportunity to assess the inner regions of SNe Ia located at the faint end of the luminosity vs. declinerate relation. Figure 6 displays the nebular spectra of SN 2007on taken on $+286 \mathrm{~d},+353 \mathrm{~d}$, and $+380 \mathrm{~d}$, as well as those of SN 2011 iv taken on $+142 \mathrm{~d},+244 \mathrm{~d}$, and $+260 \mathrm{~d}$. Late-phase spectra of both $\mathrm{SNe}$ are characterised by emission features formed by numerous blended, mostly forbidden emission lines. The strongest emission feature is around $4800 \AA$ followed by those in the ranges 4000-4500 $\AA, 5100-5400 \AA$, and 7000$8000 \AA$. The $4800 \AA$ feature is dominated by Fe III with weak contributions of Fe II, while the 5000-5500 $\AA$ feature is dominated by Fe II with contributions of Fe III (Axelrod 1980). Close inspection of the spectra of SN 2007on at $+353 d$ and $+380 d$ reveal the existence of double-peak profiles, which were first noted by Dong et al. (2015), leading them to suggest a bi-modal ${ }^{56} \mathrm{Ni}$ distribution possibly linked to a non-standard explosion scenario. A detailed spectral synthesis study exploring this option for SN 2007on and SN 2011iv will be presented in a forthcoming publication (Mazzali et al., in prep.).

Preliminary modelling of the nebular spectra has been accomplished with a non-local-thermodynamic-equilibrium (NLTE) SN nebular code (Mazzali et al. 2007). Spectral synthesis suggests ${ }^{56} \mathrm{Ni}$ masses of $0.19 M_{\odot}$ produced in SN 2007on and $0.41 M_{\odot}$ in SN 2011 iv. These values are within $\sim 23 \%$ and $5 \%$ of the values computed from the peak of the UVOIR light curves of SN 2007on and SN 2011iv, respectively. We note that the two different methods to estimate the ${ }^{56} \mathrm{Ni}$ mass have an expected scatter of $\sim 20 \%$ (see Stritzinger et al. 2006).

\section{Discussion}

\subsection{SN 2007on and SN 2011iv: transitional SNe la}

We have presented a comprehensive set of photometric and spectroscopic observations for the Type Ia SN 2007on and SN 2011 iv, both hosted by NGC 1404, and provided a detailed analysis of their data. The findings in this paper suggest that both $\mathrm{SNe}$ Ia are transitional objects, which means they have properties photometrically and spectroscopically between those of normal SNe Ia and subluminous, SN 1991bg-like SNe Ia.

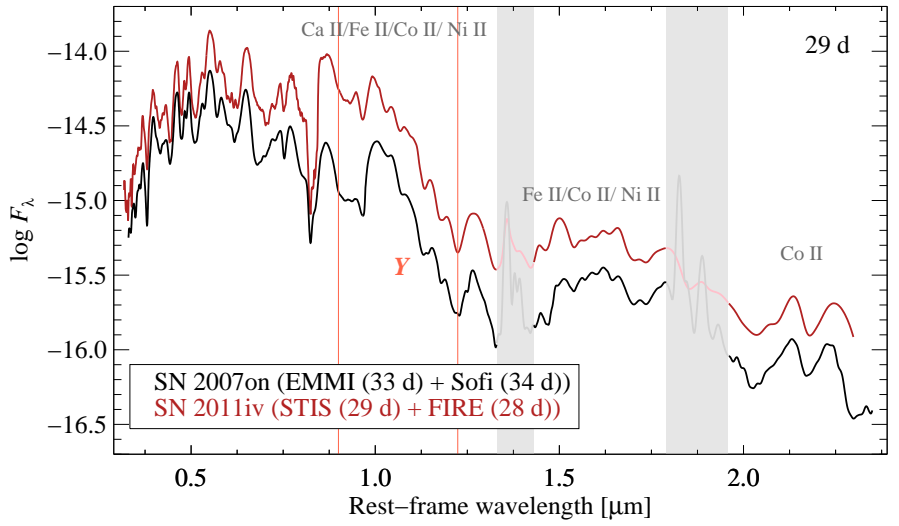

Fig. 16. Comparison of visual-wavelength and NIR spectra of SN 2007on and SN 2011iv taken around a month past maximum light. Each spectrum has been calibrated to match the broad-band photometry on $+29 \mathrm{~d}$ and smoothed for presentation purposes.

Figures 2 and 7 demonstrate that SN 2011iv is brighter than SN 2007on by $\sim 0.6 \mathrm{mag}$ in the $B$ band and $0.35 \mathrm{mag}$ in the $H$ band at maximum light. Although both objects are nearly as bright as the faintest normal SNe Ia, their optical decline rates are consistent with fast-declining, subluminous SNe Ia, with $\Delta m_{15}$ typically $\gtrsim 1.7$. Nonetheless, from a comparison of the colourstretch parameter, $s_{B V}$ (Fig. 7), we find that both SNe Ia are positioned in the luminosity vs. $s_{B V}$ relation between normal SNe Ia $\left(s_{B V} \gtrsim 0.8\right)$ and SN 1991 bg-like objects $\left(s_{B V} \lesssim 0.5\right)$.

Additionally, the NIR light curves of SN 2007on and SN 2011iv are found to peak prior to the time of $B$-band maximum, and both exhibit a secondary NIR maximum. These characteristics are consistent with normal SNe Ia and are contrary to bona fide SN 1991bg-like SNe Ia that exhibit single-peaked NIR light curves, which typically peak $\sim 2-5$ days "after" $B$-band maximum (Krisciunas et al. 2009).

To place order among the normal, transitional, and subluminous $\mathrm{SNe}$ Ia, presented in Fig. 17 is a comparison of near-maximum spectra (arranged from top down by decreasing peak luminosity) of the normal Type Ia SN 2004eo (Pastorello et al. 2007), the transitional Type Ia SNe 2011iv, iPTF13ebh (Hsiao et al. 2015), and 2007on, and the subluminous Type Ia SNe $1986 \mathrm{G}$ (Cristiani et al. 1992) and 1991bg (Turatto et al. 1996). Examination of the spectral sequence reveals that the normal and transitional objects exhibit spectral features related to the transition of doubly to singly ionised Fegroup elements, while the subluminous objects exhibit Ti II. The strength of these features and how they vary can be understood to first order by a range in photospheric temperatures (Nugent et al. 1995). Within this framework, Ti II features are present in subluminous SNe Ia characterised by cooler photospheres, while Fe II features appear with increased temperature and luminosity, followed by even higher ionisation driven by increased temperatures and the emergence of Fe III. This is consistent with the luminosity vs. decline-rate relation (e.g. Hoeflich et al. 1996), and as explained below, it is linked to the amount of ${ }^{56} \mathrm{Ni}$ produced during the explosion.

\subsection{Model comparison}

To gain a theoretically based understanding of the key differences between SN 2007on and SN 2011iv we seek guidance from a suite of spherical one-dimensional (1D) delayeddetonation (DD) explosion models of Chandrasekhar-mass 


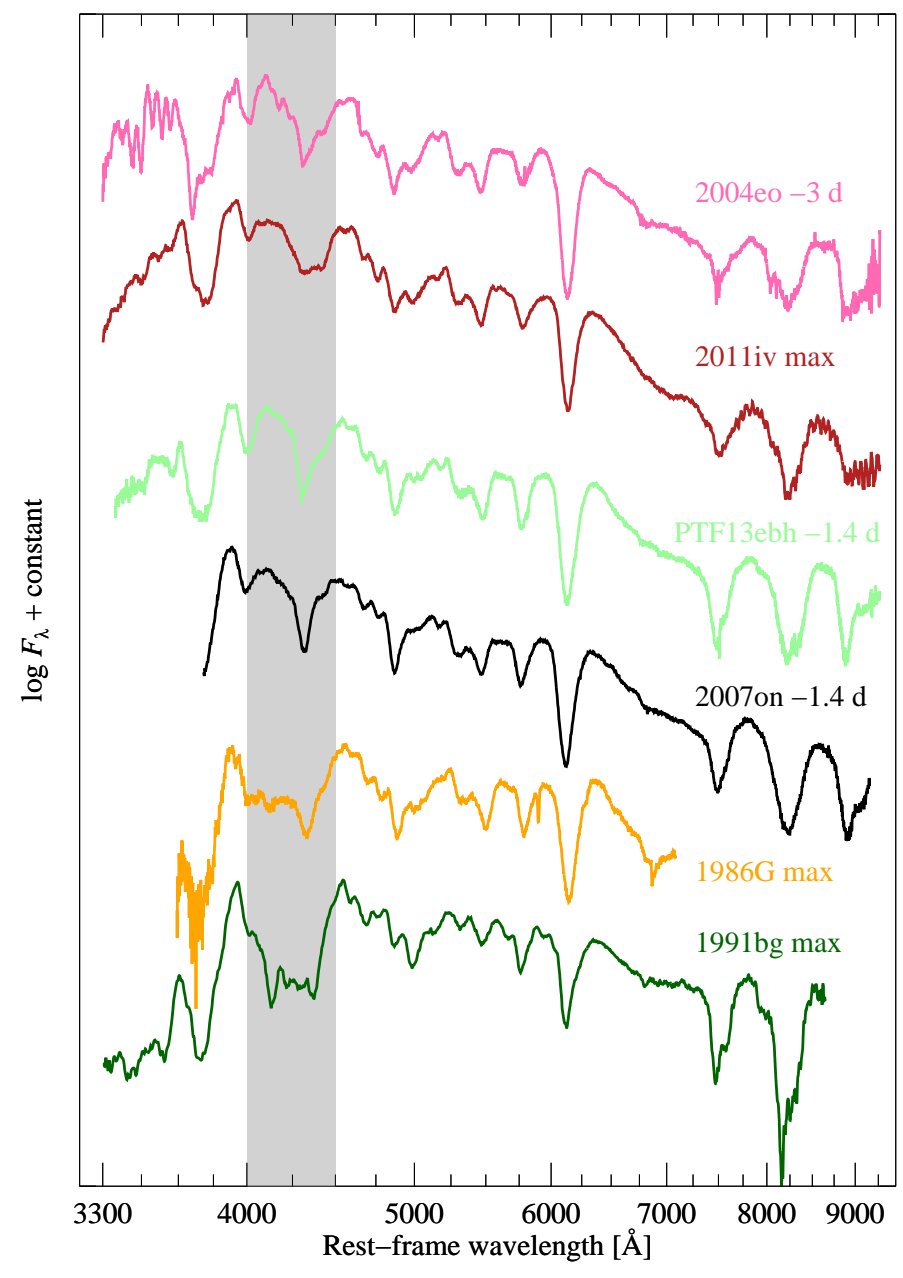

Fig. 17. Visual-wavelength spectral comparisons around maximum brightness (arranged from top to bottom by decreasing peak luminosity) of the normal SN 2004eo (Pastorello et al. 2007), SN 2011iv, iPTF13ebh (Hsiao et al. 2015), SN 2007on, SN 1986G (Cristiani et al. 1992), and SN 1991bg (Turatto et al. 1996). The spectra of SN 1986G were dereddened using the reddening parameters of $A_{V}=2.03 \mathrm{mag}$ and $R_{V}=2.57$, while spectra of SN $1991 \mathrm{bg}$ were dereddened using the parameters $A_{V}=0.22 \mathrm{mag}$ and $R_{V}=3.1$. The grey shaded region highlights the $4150 \AA$ Ti II feature that is weak in SN 1986G, prevalent in SN 1991bg, and not present in the other comparison objects (see text for discussion).

$\left(M_{\mathrm{Ch}} \approx 1.4 M_{\odot}\right)$ carbon-oxygen white dwarfs (Hoeflich et al. 2002). Spherical 1D models are used as current 3D hydrodynamical models predict significant mixing throughout the envelope which is inconsistent with observations (see Appendix C for discussion).

In standard DD models, a larger amount of ${ }^{56} \mathrm{Ni}$ is produced during the detonation burning phase compared to the deflagration burning phase (Khokhlov 1991). During the deflagration phase, 0.25 to $0.30 M_{\odot}$ of carbon-oxygen is required to be burned to lift the white dwarf from its gravitational potential. In spherically symmetric explosion models, this amount is conveniently parameterised by the transition density (hereafter $\rho_{\mathrm{tr}}$ ), which marks the density of the burning material when the laminar burning flame transitions from traveling at less than the local sound speed (a deflagration) to faster than the local sound speed (a detonation). During the deflagration phase, burning reaches nuclear statistical equilibrium (NSE), leading to the production of mostly iron-group elements. The abundances of isotopes depends on the level of neutronisation, which is specified by the electron to baryon fraction: $Y_{e}=Y_{p} /\left(Y_{p}+Y_{n}\right)$. With increasing central density (hereafter $\rho_{\mathrm{c}}$ ), electron capture shifts NSE away from the production of radioactive ${ }^{56} \mathrm{Ni}$ and toward the production of stable Fe-group elements (e.g. ${ }^{58} \mathrm{Ni}$ ). Therefore, with increasing $\rho_{\mathrm{c}}$, the abundance of stable Fe-group elements increases relative to ${ }^{56} \mathrm{Ni}$, and inevitably produces a central hole in the ${ }^{56} \mathrm{Ni}$ distribution. Alternatively, in high-metallicity white dwarfs, settling of ${ }^{22} \mathrm{Ne}$ in the core can also shift the NSE abundances away from ${ }^{56} \mathrm{Ni}$ in the central regions, but this requires very long evolutionary times on the order of five or more billion years (Bravo et al. 2011). Since the amount of ${ }^{22} \mathrm{Ne}$ is relatively limited, the size of the ${ }^{56} \mathrm{Ni}$ hole is expected to account for $\leq 0.05 M_{\odot}$ (Bildsten \& Hall 2001).

During the deflagration phase, depending on $\rho_{\mathrm{c}},{ }^{56} \mathrm{Ni}$ production can range from very little up to $\sim 0.3 M_{\odot}$ (e.g. Nomoto et al. 1984; Brachwitz et al. 2000; Hoeflich et al. 2002; Diamond et al. 2015). During the detonation phase, depending on the value of $\rho_{\mathrm{tr}},{ }^{56} \mathrm{Ni}$ production can range from very little to $\sim 0.6 M_{\odot}$ (Hoeflich et al. 2002). Therefore, the total production of ${ }^{56} \mathrm{Ni}$ in DD models can range from very little up to $\sim 0.9 M_{\odot}$, with a varying distribution of ${ }^{56} \mathrm{Ni}$ within the expanding ejecta. While in normal SNe Ia the total ${ }^{56} \mathrm{Ni}$ mass is dominated by contributions from both the deflagration and detonation phases, in subluminous $\mathrm{SNe}$ Ia the ${ }^{56} \mathrm{Ni}$ is produced primarily during the deflagration phase, leading to a ${ }^{56} \mathrm{Ni}$ distribution more centrally condensed and influenced by $\rho_{\mathrm{c}}$.

Within this well-established framework, the higher peak luminosity (and hence slower decline rate) of SN 2011 iv compared to SN 2007on is attributed to having produced more ${ }^{56} \mathrm{Ni}$, and this in turn produced the bluer colours exhibited by SN 2011iv at early times (see Fig. 8). The dependence of temperature and luminosity on the ${ }^{56} \mathrm{Ni}$ mass was described decades ago by Arnett (1982), and this relationship also drives the spectroscopic sequence plotted in Fig. 17 (see also Nugent et al. 1995). Specifically, objects with smaller amounts of ${ }^{56} \mathrm{Ni}$ will have lowertemperature photospheres leading to the presence of Ti II lines, while higher ${ }^{56} \mathrm{Ni}$ mass objects will have hotter photospheres and therefore higher-ionisation conditions leading to the presence of Fe II and or Fe III features.

As demonstrated in Fig. 8, despite SN 201 liv being brighter and bluer than SN 2007on at early epochs, between $+20 \mathrm{~d}$ and $+85 \mathrm{~d}$ SN 2011 iv appears $\sim 0.12 \pm 0.01 \mathrm{mag}$ redder than SN 2007on, and this behaviour is confirmed by the UV vs. optical colour (see Fig. 9). This rather perplexing behaviour can be explained by the progenitor white dwarf of SN 2011iv having a larger $\rho_{\mathrm{c}}$ compared to SN 2007on. As discussed above, increasing $\rho_{\mathrm{c}}$ leads to the production of more stable Fe-group elements at the expense of radioactive ${ }^{56} \mathrm{Ni}$. Less ${ }^{56} \mathrm{Ni}$ is produced in the deflagration phase owing to the higher value of $\rho_{\mathrm{c}}$. In turn, this leads to less centrally condensed ${ }^{56} \mathrm{Ni}$, less heating of the central regions of the ejecta, and (consequently) redder colours at late times.

To demonstrate the influence $\rho_{\mathrm{c}}$ has on the $B-V$ colour evolution, plotted in the inset of Fig. 8 is the $B-V$ colour evolution of SN 2007on and SN 2011iv compared to that corresponding to the best-fit DD models (see Appendix D). At early times the models match the colours quite well, while at later times the models are steeper than observed in SN 2007on and SN 2011iv. This is largely due to forbidden lines become increasingly important, and the processes associated with these transitions are difficult to model because of a lack of atomic data (see, e.g. Höflich 1995; Dessart et al. 2014; Baron et al. 2015; Telesco et al. 2015; Friesen et al. 2014, 2017). Nonetheless the 
best-fit DD models demonstrate that with increased $\rho_{\mathrm{c}}$ one obtains redder $B-V$ colours along the Lira relation, while models with reduced $\rho_{\mathrm{c}}$ exhibit bluer $B-V$ colours. Assuming a canonical value of $\rho_{\mathrm{c}}=2 \times 10^{9} \mathrm{~g} \mathrm{~cm}^{-3}$ for SN 2011iv, a $B-V$ colour offset of $0.12 \mathrm{mag}$ between models during the phases coincident with the Lira relation corresponds to a model with $\rho_{\mathrm{c}}=1$ $\times 10^{9} \mathrm{~g} \mathrm{~cm}^{-3}$ for SN 2007on. As shown in Hoeflich et al. (2017), the offset is relatively stable over the period of the Lira relation for models of different brightness. We note that a similar result was also found from the detailed analysis of the low-luminosity SN 1986G (Ashall et al. 2016b).

\subsection{Variations in luminosity}

Tentative evidence exists that SNe Ia residing in early-type galaxies exhibit smaller Hubble scatter than SNe Ia hosted in late-type galaxies (Sullivan et al. 2003). This is thought to be driven by (i) the progenitor stars being older and spanning a smaller mass range; and (ii) dust extinction being less significant in early-type hosts compared with late-type hosts. Some high-redshift experiments have therefore been designed to target early-type galaxies in distant clusters.

With SN 2007on and SN 20011iv being both located in the same galaxy, they offer a rare opportunity to test the assertion that $\mathrm{SNe}$ Ia located in early-type hosts provide minimal dispersion in their peak luminosities. However, as we have shown in Sect. 4, even after correcting for colour stretch and colour (extinction), both objects exhibit significantly different peak absolute magnitudes. This is in contrast to the three normal $\left(\Delta m_{15}(B) \approx 1.1-1.2 \mathrm{mag}\right) \mathrm{SNe}$ Ia hosted in the early-type galaxy Fornax A, whose distances were found to be consistent at the 3\% level (Stritzinger et al. 2010). Therefore, the significant discrepancy between the distances of SN 2007on and SN 2011iv serves as a cautionary tale for the use of transitional $\mathrm{SNe}$ Ia located in early-type hosts in the quest to measure cosmological parameters. The implications of this finding are important in cosmology; for example, more than half of the $\mathrm{SNe}$ Ia used by Suzuki et al. (2012) to constrain the high-redshift $(z>1)$ end of their Hubble diagram are fast-declining SNe (i.e. objects best described by the SALT parameter $x_{1}<-1$, or equivalently $\left.\Delta m_{15}(B)>1.4 \mathrm{mag}\right)$.

As described above, within the context of the DD $M_{\mathrm{Ch}}$ models employed in this study, the ${ }^{56} \mathrm{Ni}$ production depends both on $\rho_{\mathrm{c}}$ and $\rho_{\mathrm{tr}}$. While $\rho_{\mathrm{tr}}$ is the primary driver of the luminosity vs. decline-rate relation, $\rho_{\mathrm{c}}$ plays an important role for the fasterdeclining SNe Ia which produce smaller amounts of radioactive ${ }^{56} \mathrm{Ni}$ as compared to normal-luminosity $\mathrm{SNe}$ Ia. In $\mathrm{SNe}$ with the same $\Delta m_{15}(B)$ (so-called "twins"), this secondary parameter can lead to changes in the peak brightness of 0.05 mag for normalbrightness $\mathrm{SNe}$ Ia, and by up to $0.7 \mathrm{mag}$ in the case of twin subluminous SNe Ia (Hoeflich et al. 2017).

Höflich et al. (2010) studied the variation in brightness between the peak and the tail (roughly the brightness at day +40 ) caused by variations in progenitor mass, metallicity, accretion rate, $\dot{M}$, and central density. The effects of variations in $\rho_{\mathrm{c}}$ are further elucidated in Fig. 7 of Hoeflich et al. (2017), which shows the variation of heating from gamma-ray deposition as a function of central concentration of radioactive nickel. This more central heating in lower- $\rho_{\mathrm{c}} \mathrm{SNe}$ leads to a hotter central region and therefore bluer colours, with a variation in $B-V$ of up to 0.2 mag. $\mathrm{SNe}$ of comparable central densities have similar Lira relations. The differences in SN 2007on and SN 2011iv lead to expected variations in $\rho_{\mathrm{c}}$ of a factor of $\sim 2$ (see Sect. 6.2). For this density variation, we expect SN 2007on would be brighter at maximum by about 0.18 mag than a model with the same $\rho_{\mathrm{c}}$ of SN 2011iv. These modifications bring the distance of both $\mathrm{SNe}$ to within $\sim 0.1 \mathrm{mag}$. In light of these results, our findings suggest that observations extended to at least +40 days are required to break the degeneracy between $\rho_{\mathrm{c}}$ and the luminosity vs. declinerate relation in fast-declining SNe Ia. Consequently, future SN Ia cosmological experiments should consider obtaining photometry at phases coincident with the Lira relation.

\section{Summary and conclusions}

We have presented a comprehensive set of photometric and spectroscopic observations - spanning from UV through NIR wavelengths - of the transitional Type Ia SNe 2007on and 2011iv. The detailed observational dataset combined with modelled comparisons, and the fact both objects were located in the same host galaxy NGC 1404, allowed us to obtain insights on their progenitors and their ability to serve as distance indicators. The main findings of this study are as follows.

- The transitional Type Ia SNe 2007on and 2011iv exhibit spectral and light-curve properties consistent with being an extension of the normal SN Ia population, and are not consistent with the observed properties of subluminous SN 1991bg-like SNe Ia.

- The difference in their peak luminosities is caused by differences in their ${ }^{56} \mathrm{Ni}$ production. This drives the differences in their $B-V$ colours around maximum brightness, as well as subtle differences in their spectroscopic properties.

- The $B-V$ colour evolution of SN 2011iv is found to transition from being bluer to redder than SN 2007on between maximum brightness and several weeks later. We suggest that this behaviour is linked to the progenitor of SN 2011iv having a higher $\rho_{\mathrm{c}}$ than the progenitor of SN 2007on. With a higher $\rho_{\mathrm{c}}$, SN $2011 \mathrm{iv}$ produced more stable ${ }^{58} \mathrm{Ni}$ in the centre of its ejecta, leading to the formation of a central hole in the distribution of ${ }^{56} \mathrm{Ni}$. This ultimately leads to less energy deposition in the central region of the ejecta and hence the redder colours observed in SN 2011iv as compared with SN 2007on along the Lira relation. The colour difference of $\sim 0.12$ mag suggests that $\rho_{\mathrm{c}}$ in SN 2011iv was a factor of two larger than in SN 2007on.

- An analysis of the $B$ - and $H$-band distance estimates of SN 2007on and SN 2011iv reveals relative differences between the two objects of $\sim 14 \%$ and $\sim 9 \%$, respectively. These differences serve as a warning in the use of transitional $\mathrm{SNe}$ Ia in future efforts to measure cosmological parameters. New observational campaigns centred around lowluminosity SNe Ia should place an effort on obtaining data extending out to two to three months past maximum light, in order to account for luminosity discrepancies related to $\rho_{\mathrm{c}}$ differences in their progenitors.

Acknowledgements. This work is based in part on observations made at the Las Campanas Observatory, including the $6.5 \mathrm{~m}$ Magellan Telescope. It is also based in part on spectra collected at the European Organization for Astronomical Research in the Southern Hemisphere, Chile (ESO Programmes 184.D-1151, 184.D-1152, 088.D-0222, 184.D-1140, 080.A-0516, 080.C-0833); the $8.1 \mathrm{~m}$ Gemini-S Telescope (Program GS-2011B-Q-1); the Nordic Optical Telescope (Program 44-024); and the NASA/ESA Hubble Space Telescope (GO-12592), obtained at the Space Telescope Science Institute (STScI), which is operated by the Association of Universities for Research in Astronomy, Inc., under National Aeronautics and Space Administration (NASA) contract NAS 5-26555 (Programs GO-12298, GO-12582, GO-12592, GO-13286, and GO-13646). Swift spectroscopic observations were performed under program GI-5080130. Some of the data presented herein were obtained at the W. M. Keck Observatory, which 
is operated as a scientific partnership among the California Institute of Technology, the University of California, and NASA; the observatory was made possible by the generous financial support of the W. M. Keck Foundation. We thank J. Silverman for useful discussions pertaining to the HST observations and E. Newton for obtaining some NIR spectroscopic observations. Supernova research at Aarhus University is supported in part by a Sapere Aude Level 2 grant funded by the Danish Agency for Science and Technology and Innovation, and the Instrument Centre for Danish Astrophysics (IDA). C. Gall also acknowldeges support from the Carlsberg Foundation. M. Stritzinger is also supported by a research grant (13261) from VILLUM FONDEN. The CSP-I is supported by the US National Science Foundation (NSF) under grants AST-0306969, AST-0607438, AST-1008343, AST-1613426, AST-1613455, and AST-1613472. A portion of the work presented here was done at the Aspen Center for Physics, which is supported by US NSF grant PHY-1066293. A. V. Filippenko is grateful for financial assistance from US NSF grant AST-1211916, the TABASGO Foundation, the Christopher R. Redlich Fund, and the Miller Institute for Basic Research in Science (U.C. Berkeley); he also acknowledges HST grants GO-13286, GO-13646, and AR-14295 from STScI, which is operated by AURA under NASA contract NAS 5-26555. Filippenko's work was conducted in part at the Aspen Center for Physics, which is supported by NSF grant PHY-1607611; he thanks the Center for its hospitality during the neutron stars workshop in June and July 2017. G. Pignata acknowledges support provided by the Millennium Institute of Astrophysics (MAS) through grant IC120009 of the Programa Iniciativa Cientifica Milenio delMinisterio de Economia, Fomento y Turismo de Chile. N. Elias de la Rosa acknowledges financial support by the 1994 PRIN-INAF 2014 (project "Transient Universe: unveiling new types of stellar explosions with PESSTO") and by MIUR PRIN 2010-2011, "The dark Universe and the cosmic evolution of baryons: from current surveys to Euclid". S. Benetti is partially supported by the PRIN-INAF 2014 project "Transient Universe: unveiling new types of stellar explosions with PESSTO". P. Hoeflich acknowledges financial support by the grant 1715133 by the National Science Foundation entitled "Signatures of Type Ia Supernovae Explosions and their Cosmological Implications". R. J Foley is supported by NASA under Contract No. NNG16PJ34C issued through the WFIRST Science Investigation Teams Programme. The UCSC group is supported in part by NASA grant 14-WPS14-0048, US NSF grant AST-1518052, and from fellowships from the Alfred P. Sloan Foundation and the David and Lucile Packard Foundation. We thank the STScI staff for accommodating ou target-of-opportunity programs. A. Armstrong, R. Bohlin, S. Holland, S. Meyett D. Sahnow, P. Sonnentrucker, and D. Taylor were critical for the execution of these programs. Finally, we are grateful to N. Gehrels and the Swift team for executing our programme promptly.

\section{References}

Albrecht, A., Bernstein, G., Cahn, R., et al. 2006, ArXiv e-prints [arXiv: astro-ph/0609591]

Arnett, W. D. 1982, ApJ, 253, 785

Ashall, C., Mazzali, P., Bersier, D., et al. 2014, MNRAS, 445, 4427

Ashall, C., Mazzali, P., Sasdelli, M., \& Prentice, S. J. 2016a, MNRAS, 460, 3529

Ashall, C., Mazzali, P. A., Pian, E., \& James, P. A. 2016b, MNRAS, 463, 1891

Ashall, C., Mazzali, P. A., Stritzinger, M., et al. 2018, MNRAS, submitted

Axelrod, T. S. 1980, Ph.D. Thesis, California Univ., Santa Cruz, USA

Baron, E., Hoeflich, P., Friesen, B., et al. 2015, MNRAS, 454, 2549

Barone-Nugent, R. L., Lidman, C., Wyithe, J. S. B., et al. 2012, MNRAS, 425, 1007

Ben-Ami, S., Hachinger, S., Gal-Yam, A., et al. 2015, ApJ, 803, 40

Betoule, M., Kessler, R., Guy, J., et al. 2014, A\&A, 568, A22

Bildsten, L., \& Hall, D. M. 2001, ApJ, 549, L219

Blakeslee, J. P., Jordán, A., Mei, S., et al. 2009, ApJ, 694, 556

Blondin, S., Matheson, T., Kirshner, R. P., et al. 2012, AJ, 143, 126

Brachwitz, F., Dean, D. J., Hix, W. R., et al. 2000, ApJ, 536, 934

Branch, D. 1987, ApJ, 316, L81

Branch, D., \& Venkatakrishna, K. L. 1986, ApJ, 306, L21

Branch, D., Dang, L. C., Hall, N., et al. 2006, PASP, 118, 560

Bravo, E., Althaus, L. G., García-Berro, E., \& Domínguez, I. 2011, A\&A, 526, A26

Breeveld, A. A., Landsman, W., Holland, S. T., et al. 2011, eds. J. E. McEnery, J. L. Racusin, \& N. Gehrels, in AIP Conf. Ser. 1358, 373

Brown, P. J., Breeveld, A. A., Holland, S., Kuin, P., \& Pritchard, T. 2014, Ap\&SS 354,89

Brown, P. J., Baron, E., Milne, P., Roming, P. W. A., \& Wang, L. 2015, ApJ, 809, 37

Bufano, F., Immler, S., Turatto, M., et al. 2009, ApJ, 700, 1456

Burns, C. R., Stritzinger, M., Phillips, M. M., et al. 2011, AJ, 141, 19

Burns, C. R., Stritzinger, M., Phillips, M. M., et al. 2014, ApJ, 789, 32

Burrows, D. N., Hill, J. E., Nousek, J. A., et al. 2005, Space Sci. Rev., 120, 165

Calder, A. C., Plewa, T., Vladimirova, N., Lamb, D. Q., \& Truran, J. W. 2004, ArXiv e-prints [arXiv: astro-ph/0405162]
Castelli, F., \& Kurucz, R. L. 2003, in Modelling of Stellar Atmospheres, eds. N. Piskunov, W. W. Weiss, \& D. F. Gray, IAU Symp., 210, A20

Ciaraldi-Schoolmann, F., Seitenzahl, I. R., \& Röpke, F. K. 2013, A\&A, 559, A117

Contreras, C., Hamuy, M., Phillips, M. M., et al. 2010, AJ, 139, 519

Corwin, Jr., H. G., Buta, R. J., \& de Vaucouleurs, G. 1994, AJ, 108, 2128

Cristiani, S., Cappellaro, E., Turatto, M., et al. 1992, A\&A, 259, 63

Dessart, L., Hillier, D. J., Blondin, S., \& Khokhlov, A. 2014, MNRAS, 441, 3249

Diamond, T. R., Hoeflich, P., \& Gerardy, C. L. 2015, ApJ, 806, 107

Domínguez, I., Höflich, P., \& Straniero, O. 2001, Nucl. Phys. A, 688, 21

Dong, S., Katz, B., Kushnir, D., \& Prieto, J. L. 2015, MNRAS, 454, L61

Drescher, C., Parker, S., Brimacombe, J., Noguchi, T., \& Nakano, S. 2011, Central Bureau Electron. Tel., 2940, 1

Fesen, R., Hoeflich, P., \& Hamilton, A. 2016, in Supernova Remnants: An Odyssey in Space after Stellar Death, 115

Fesen, R. A., Hoeflich, P. A., Hamilton, A. J. S., et al. 2007, ApJ, 658, 396

Fesen, R. A., Höflich, P. A., \& Hamilton, A. J. S. 2015, ApJ, 804, 140

Filippenko, A. V. 1997, ARA\&A, 35, 309

Filippenko, A. V., Richmond, M. W., Branch, D., et al. 1992a, AJ, 104, 1543

Filippenko, A. V., Richmond, M. W., Matheson, T., et al. 1992b, ApJ, 384, L15

Fink, M., Kromer, M., Seitenzahl, I. R., et al. 2014, MNRAS, 438, 1762

Fitzpatrick, E. L. 1999, PASP, 111, 63

Folatelli, G. 2004, New Astron. Rev., 48, 623

Folatelli, G., Phillips, M. M., Burns, C. R., et al. 2010, AJ, 139, 120

Folatelli, G., Morrell, N., Phillips, M. M., et al. 2013, ApJ, 773, 53

Foley, R. J., Filippenko, A. V., \& Jha, S. W. 2008, ApJ, 686, 117

Foley, R. J., Challis, P. J., Filippenko, A. V., et al. 2012a, ApJ, 744, 38

Foley, R. J., Kromer, M., Howie Marion, G., et al. 2012b, ApJ, 753, L5

Foley, R. J., Pan, Y.-C., Brown, P., et al. 2016, MNRAS, 461, 1308

Friesen, B., Baron, E., Wisniewski, J. P., et al. 2014, ApJ, 792, 120

Friesen, B., Baron, E., Parrent, J. T., et al. 2017, MNRAS, submitted

Gal-Yam, A., Simon, J., Klotz, A., \& Rosolowsky, E. 2007, ATel, 1263, 1

Gamezo, V. N., Khokhlov, A. M., Oran, E. S., Chtchelkanova, A. Y., \&

Rosenberg, R. O. 2003, Science, 299, 77

Gamezo, V. N., Khokhlov, A. M., \& Oran, E. S. 2005a, ApJ, 623, 337

Gamezo, V. N., Khokhlov, A. M., \& Oran, E. S. 2005b, ApJ, 623, 337

Ganeshalingam, M., Li, W., Filippenko, A. V., et al. 2010, ApJS, 190, 418

Garavini, G., Folatelli, G., Nobili, S., et al. 2007, A\&A, 470, 411

Garnavich, P. M., Bonanos, A. Z., Krisciunas, K., et al. 2004, ApJ, 613, 1120

Gerardy, C. L., Hoeflich, P., Fesen, R. A., et al. 2004, ApJ, 607, 391

Graham, A. W., Colless, M. M., Busarello, G., Zaggia, S., \& Longo, G. 1998,

A\&AS, 133, 325

Hachinger, S., Mazzali, P. A., \& Benetti, S. 2006, MNRAS, 370, 299

Hachinger, S., Mazzali, P. A., Tanaka, M., Hillebrandt, W., \& Benetti, S. 2008, MNRAS, 389, 1087

Hamuy, M., Folatelli, G., Morrell, N. I., et al. 2006, PASP, 118, 2

Hoeflich, P. 2006, Nucl. Phys. A, 777, 579

Hoeflich, P., Khokhlov, A., Wheeler, J. C., et al. 1996, ApJ, 472, L81

Hoeflich, P., Wheeler, J. C., \& Thielemann, F. K. 1998, ApJ, 495, 617

Hoeflich, P., Gerardy, C. L., Fesen, R. A., \& Sakai, S. 2002, ApJ, 568, 791

Hoeflich, P., Hsiao, E. Y., Ashall, C., et al. 2017, ApJ, 846, 58

Höflich, P. 1995, ApJ, 443, 89

Höflich, P., Gerardy, C. L., Nomoto, K., et al. 2004, ApJ, 617, 1258

Höflich, P., Krisciunas, K., Khokhlov, A. M., et al. 2010, ApJ, 710, 444

Hoyle, F., \& Fowler, W. A. 1960, ApJ, 132, 565

Hristov, B., Collins, D. C., Hoeflich, P., \& Weatherford, C. 2016, in AAS Meeting Abstracts, 227, 237.16

Hsiao, E. Y., Conley, A., Howell, D. A., et al. 2007, ApJ, 663, 1187

Hsiao, E. Y., Marion, G. H., Phillips, M. M., et al. 2013, ApJ, 766, 72

Hsiao, E. Y., Burns, C. R., Contreras, C., et al. 2015, A\&A, 578, A9

Iwamoto, K., Brachwitz, F., Nomoto, K., et al. 1999, ApJS, 125, 439

Kasen, D., \& Woosley, S. E. 2007, ApJ, 656, 661

Kelly, P. L., Filippenko, A. V., Burke, D. L., et al. 2015, Science, 347, 1459

Khokhlov, A. M. 1991, A\&A, 245, 114

Khokhlov, A. M. 1995, ApJ, 449, 695

Khokhlov, A. M. 2000, ArXiv e-prints [arXiv:astro-ph/0008463]

Khokhlov, A. M., Oran, E. S., \& Wheeler, J. C. 1997, ApJ, 478, 678

Kirshner, R. P., Jeffery, D. J., Leibundgut, B., et al. 1993, ApJ, 415, 589

Krisciunas, K., Marion, G. H., Suntzeff, N. B., et al. 2009, AJ, 138, 1584

Kromer, M., \& Sim, S. A. 2009, MNRAS, 398, 1809

Landolt, A. U. 1992, AJ, 104, 372

Leibundgut, B., Kirshner, R. P., Phillips, M. M., et al. 1993, AJ, 105, 301

Leloudas, G., Stritzinger, M. D., Sollerman, J., et al. 2009, A\&A, 505, 265

Lentz, E. J., Baron, E., Branch, D., Hauschildt, P. H., \& Nugent, P. E. 2000, ApJ, 530,966 
Lira, P. 1996, Master's thesis, Univ. Chile

Lisewski, A. M., Hillebrandt, W., Woosley, S. E., Niemeyer, J. C., \& Kerstein, A. R. 2000, ApJ, 537, 405

Liu, M. C., Graham, J. R., \& Charlot, S. 2002, ApJ, 564, 216

Livne, E. 1999, ApJ, 527, L97

Livne, E., \& Arnett, D. 1995, ApJ, 452, 62

Livne, E., Asida, S., \& Hoeflich, P. 2005, ApJ, 632, 443

Loewenstein, M., Mushotzky, R. F., Tamura, T., et al. 1994, ApJ, 436, L75

Machacek, M., Dosaj, A., Forman, W., et al. 2005, ApJ, 621, 663

Maeda, K., Benetti, S., Stritzinger, M., et al. 2010, Nature, 466, 82

Mandel, K. S., Narayan, G., \& Kirshner, R. P. 2011, ApJ, 731, 120

Masters, K. L., Jordán, A., Côté, P., et al. 2010, ApJ, 715, 1419

Mazzali, P. A. 2000, A\&A, 363, 705

Mazzali, P. A., Foley, R. J., Deng, J., et al. 2007, ApJ, 661, 892

Mazzali, P. A., Sullivan, M., Hachinger, S., et al. 2014, MNRAS, 439, 1959

Milne, P. A., Brown, P. J., Roming, P. W. A., et al. 2010, ApJ, 721, 1627

Morrell, N., Folatelli, G., \& Stritzinger, M. 2007, Central Bureau Electron. Tel., 1131,1

Motohara, K., Maeda, K., Gerardy, C. L., et al. 2006, ApJ, 652, L101

Narayan, G., Rest, A., Tucker, B. E., et al. 2016, ApJS, 224, 3

Niemeyer, J. C., \& Hillebrandt, W. 1995a, ApJ, 452, 779

Niemeyer, J. C., \& Hillebrandt, W. 1995b, ApJ, 452, 769

Niemeyer, J. C., Hillebrandt, W., \& Woosley, S. E. 1996, ApJ, 471, 903

Noguchi, T., Nakano, S., Chen, J., et al. 2011, Central Bureau Electron. Tel., 2940, 1

Nomoto, K., Thielemann, F.-K., \& Yokoi, K. 1984, ApJ, 286, 644

Nugent, P., Phillips, M., Baron, E., Branch, D., \& Hauschildt, P. 1995, ApJ, 455, L147

Nugent, P. E., Sullivan, M., Cenko, S. B., et al. 2011, Nature, 480, 344

Pan, Y.-C., Foley, R. J., Kromer, M., et al. 2015, MNRAS, 452, 4307

Pastorello, A., Mazzali, P. A., Pignata, G., et al. 2007, MNRAS, 377, 1531

Penney, R., \& Hoeflich, P. 2014, ApJ, 795, 84

Persson, S. E., Murphy, D. C., Krzeminski, W., Roth, M., \& Rieke, M. J. 1998 AJ, 116, 2475

Phillips, M. M. 1993, ApJ, 413, L105

Phillips, M. M., Phillips, A. C., Heathcote, S. R., et al. 1987, PASP, 99, 592

Phillips, M. M., Wells, L. A., Suntzeff, N. B., et al. 1992, AJ, 103, 1632

Phillips, M. M., Lira, P., Suntzeff, N. B., et al. 1999, AJ, 118, 1766

Phillips, M. M., Simon, J. D., Morrell, N., et al. 2013, ApJ, 779, 38

Plewa, T., Calder, A. C., \& Lamb, D. Q. 2004, ApJ, 612, L37

Pollas, C., \& Klotz, A. 2007, Central Bureau Electron. Tel., 1121, 1

Reinecke, M., Hillebrandt, W., \& Niemeyer, J. C. 1999, A\&A, 347, 739

Remming, I. S., \& Khokhlov, A. M. 2014, ApJ, 794, 87

Rheault, J.-P., DePoy, D. L., Behm, T. W., et al. 2010, in Ground-based and Airborne Instrumentation for Astronomy III, Proc. SPIE, 7735, 773564
Riess, A. G., Macri, L. M., Hoffmann, S. L., et al. 2016, ApJ, 826, 56 Roelofs, G., Bassa, C., Voss, R., \& Nelemans, G. 2008, MNRAS, 391, 290 Roming, P. W. A., Kennedy, T. E., Mason, K. O., et al. 2005, Space Sci. Rev., 120,95

Röpke, F. K., Hillebrandt, W., Niemeyer, J. C., \& Woosley, S. E. 2006, A\&A, 448, 1

Ruiz-Lapuente, P., Cappellaro, E., Turatto, M., et al. 1992, ApJ, 387, L33

Sauer, D. N., Mazzali, P. A., Blondin, S., et al. 2008, MNRAS, 391, 1605

Schlafly, E. F., \& Finkbeiner, D. P. 2011, ApJ, 737, 103

Schlegel, D. J., Finkbeiner, D. P., \& Davis, M. 1998, ApJ, 500, 525

Skibba, R. A., Engelbracht, C. W., Dale, D., et al. 2011, ApJ, 738, 89

Smartt, S. J., Valenti, S., Fraser, M., et al. 2015, A\&A, 579, A40

Smith, J. A., Tucker, D. L., Kent, S., et al. 2002, AJ, 123, 2121

Srivastav, S., Anupama, G. C., Sahu, D. K., \& Ravikumar, C. D. 2017, MNRAS, 466, 2436

Steer, I., Madore, B. F., Mazzarella, J. M., et al. 2017, AJ, 153, 37

Sternberg, A., Gal-Yam, A., Simon, J. D., et al. 2011, Science, 333, 856

Sternberg, A., Gal-Yam, A., Simon, J. D., et al. 2014, MNRAS, 443, 1849

Stritzinger, M., \& Leibundgut, B. 2005, A\&A, 431, 423

Stritzinger, M., Mazzali, P. A., Sollerman, J., \& Benetti, S. 2006, A\&A, 460, 793

Stritzinger, M., Burns, C. R., Phillips, M. M., et al. 2010, AJ, 140, 2036

Stritzinger, M., Morrell, N., \& Foley, R. J. 2011a, Central Bureau Electron. Tel., 2940, 3

Stritzinger, M. D., Phillips, M. M., Boldt, L. N., et al. 2011b, AJ, 142, 156

Stritzinger, M. D., Valenti, S., Hoeflich, P., et al. 2015, A\&A, 573, A2

Stubbs, C. W., \& Brown, Y. J. 2015, Mod. Phys. Lett. A, 30, 1530030

Sullivan, M., Ellis, R. S., Aldering, G., et al. 2003, MNRAS, 340, 1057

Suzuki, N., Rubin, D., Lidman, C., et al. 2012, ApJ, 746, 85

Taubenberger, S., Hachinger, S., Pignata, G., et al. 2008, MNRAS, 385, 75

Telesco, C. M., Höflich, P., Li, D., et al. 2015, ApJ, 798, 93

Timmes, F. X., Brown, E. F., \& Truran, J. W. 2003, ApJ, 590, L83

Tonry, J. L. 1991, ApJ, 373, L1

Turatto, M., Benetti, S., Cappellaro, E., et al. 1996, MNRAS, 283, 1

Uenishi, T., Nomoto, K., \& Hachisu, I. 2003, Nucl. Phys. A, 718, 623

Vogt, S. S., Allen, S. L., Bigelow, B. C., et al. 1994, in Instrumentation in Astronomy VIII, eds. D. L. Crawford, \& E. R. Craine, Proc. SPIE, 2198, 362

Voss, R., \& Nelemans, G. 2008, Nature, 451, 802

Walker, E. S., Hachinger, S., Mazzali, P. A., et al. 2012, MNRAS, 427, 103

Yoon, S.-C., \& Langer, N. 2004, in Cosmic explosions in three dimensions, eds. P. Hoeflich, P. Kumar, \& J. C. Wheeler, 94

Yoon, S.-C., Langer, N., \& Scheithauer, S. 2004, A\&A, 425, 217

Zel'Dovich, Y. B., Librovich, V. B., Makhviladze, G. M., \& Sivashinskil, G. I. 1970, J. Appl. Mech. Tech. Phys., 11, 264 


\section{Appendix A: The UV flux ratio}

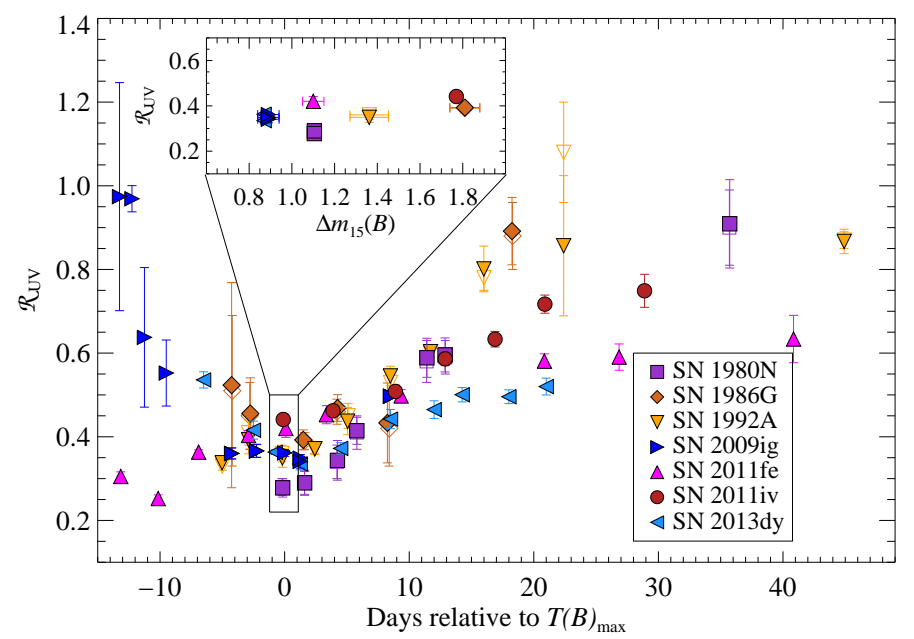

Fig. A.1. Temporal evolution of the UV ratio, $\mathcal{R}_{\mathrm{UV}}$. The $\mathcal{R}_{\mathrm{UV}}$ values for SN 2011iv between $0 \mathrm{~d}$ and $+29 \mathrm{~d}$ are shown as red filled circles. For comparison, we calculated the $\mathcal{R}_{\mathrm{UV}}$ for SN $1980 \mathrm{~N}$ and SN 1986G (Foley et al. 2008), SN 1992A (Kirshner et al. 1993), SN 2009ig (Foley et al. 2012a), and for SN 2011fe and SN 2013dy (Pan et al. 2015). Results are shown as solid orange, purple, yellow, and dark-blue symbols, respectively. Additionally, the derived $\mathcal{R}_{\mathrm{UV}}$ from Foley et al. (2008) for SN 1986G, SN 1980N, and SN 1992A are presented as open symbols consisting of the same colour and shape. The small differences arise from different assumptions about redshift and host-galaxy reddening, as described in Appendix A.

To calculate the UV flux ratio, $\mathcal{R}_{\mathrm{UV}}$, we adopt the definition of Foley et al. (2008) as $\mathcal{R}_{\mathrm{UV}}=f_{\lambda}(2770 \AA) / f_{\lambda}(2900 \AA)$. This is done for both SN 2011iv and other SNe Ia with available UV spectra including SN 1980N, SN 1986G, SN 1992A (Foley et al. 2008; Kirshner et al. 1993), SN 2009ig (Foley et al. 2012a), SN 2011fe (Mazzali et al. 2014), and SN 2013dy (Pan et al. 2015). To compute $\mathcal{R}_{\mathrm{UV}}$, all of the spectra were corrected for extinction using IDL routines equipped with the standard extinction-curve parameterisation of Fitzpatrick (1999). Milky Way reddening values and the host-galaxy recessional velocities are taken from NED. For SN 1986G, we adopt host-galaxy reddening parameters of $A_{V}=2.03 \mathrm{mag}$ and $R_{V}=2.57$ (Phillips et al. 2013).

Figure A.1 displays the temporal evolution of $\mathcal{R}_{\mathrm{UV}}$ for the selected sample of SNe Ia, which are characterised by different values of $\Delta m_{15}$ and host-galaxy properties. Seemingly, $\mathcal{R}_{\mathrm{UV}}$ does not follow a simple trend with epoch, though it appears that for all SNe Ia $\mathcal{R}_{\mathrm{UV}}$ increases between $0 \mathrm{~d}$ and $+40 \mathrm{~d}$. However, prior to $B$-band maximum brightness, $\mathcal{R}_{\mathrm{UV}}$ either increases or decreases over time for the SN Ia sample. The inset of Fig. A.1 provides a close-up view of the epoch around $B$-band maximum $( \pm 1 \mathrm{~d})$ and indicates that $\mathcal{R}_{\mathrm{UV}}$ of the selected SNe Ia does not follow a clear trend with $\Delta m_{15}$.

Additionally, also compared in Fig. A.1 are $\mathcal{R}_{\mathrm{UV}}$ values of SN 1980N, SN 1992A, SN 1986G, and those of Foley et al. (2008) computed using the same set of data. While the results overall agree, there are small differences owing to different assumed values of either reddening or host-galaxy recession velocity. As also discussed in the literature (e.g. Walker et al. 2012), $\mathcal{R}_{\mathrm{UV}}$ is not a broad-band colour measurement, and it may therefore be sensitive to various issues pertaining to both the quality of the data and data analysis, but also environmental and or intrinsic differences.

\section{Appendix B: Analysis of visual-wavelength spectroscopy}

\section{B.1. Optical pseudo-equivalent width}

In analogy to the UV spectral region, here we quantify the properties of the most prominent optical absorption features by measuring the $\mathrm{pEW}$. We assess the $\mathrm{pEW}$ of eight features in a similar way as outlined in Sect. 5.1.2, adopting the definition and naming convention as described by Folatelli et al. (2013, see their Table 4). In short, the specific pEWs are as follows: pW1 (Ca II H\&K), pW2 (Si II 14130), pW3 (Mg II), pW4 (Fe II), pW5 (S II W), pW6 (Si II 25972), pW7 (Si II 26355), and pW8 (Ca II NIR triplet). We note that for SN 2007on and SN 2011iv, the pW3 (Mg II) is a blend dominated by either Mg II $\lambda 4481$ or Si III $\lambda 4560$, and pW4 (Fe II) is shaped by S II $\lambda 5032$, Si II $\lambda 5055$, and Fe II $\lambda 5083$.

Figure B.1 presents the temporal evolution of the $\mathrm{pEW}$ values for all eight features as measured from the spectroscopic sequences of SN 2007on and SN 2011iv (see Tables F.6 and F.7). Additionally, Fig. B.1 displays the pEW values of SN 1986G, SN 1991bg, and iPTF13ebh, as well as the average $\mathrm{pEW}$ values (and their associated $1 \sigma$ dispersion) as measured from the CSP-I SN Ia sample (Folatelli et al. 2013).

Inspection of Fig. B.1 reveals that fast-declining SNe Ia show very similar $\mathrm{pEW}$ values across all epochs, although the $\mathrm{pEW}$ values of pW4 are somewhat lower in SN 2007on. The measured $\mathrm{pEW}$ values of the fast-declining SNe Ia are found to be largely similar with the average $\mathrm{pEW}$ values of the normal SNe Ia, except in the case of pW1 (Ca II H\&K) and pW5 (S II W), which are both consistently below the mean sample values. On the other hand, the pW6 (Si II 25972) pEW values are larger than what is found in normal SNe Ia, and this is also consistent with observations of other fast-declining SNe Ia (Hachinger et al. 2006). This phenomenon is a consequence of the increasing Si III to Si II recombination rate that is caused by a decrease in temperature, leading to a more prevalent Si II $\lambda 5972$ feature (see Hachinger et al. 2008). Finally, the measured pEW values of pW2 (Si II 24130) and pW7 (Si II 26355) are found to be in agreement with the average $\mathrm{pEW}$ values of the normal SNe Ia, while for $\mathrm{pW} 3$ (Mg II) and pW4 (Fe II) the fast-declining objects have larger $\mathrm{pEW}$ values until about $+10 \mathrm{~d}$ to $+20 \mathrm{~d}$.

To categorise the spectroscopic diversity of $\mathrm{SNe}$ Ia, Branch et al. (2006) introduced a classification scheme based on the pEW values of pW6 (Si II 25972) and pW7 (Si II 26355) at maximum light. There are four subclasses defined: the "cool" (CL), the "broad line" (BL), the "shallow silicon" (SS), and the "core normal" $(\mathrm{CN})$. Adopting the definition as outlined by Blondin et al. (2012) and Folatelli et al. (2013), SN 2007on and SN 2011iv are both clearly CL SN Ia.

\section{B.2. Optical line velocity}

Here we present measurements of the Doppler velocity at maximum absorption of the most prominent ions. This provides an estimate of the velocity distribution of the various line-forming regions of the ejecta. Measurements were obtained by fitting a Gaussian function to the absorption profiles using a custommade IDL program.

Figure B.2 displays the Doppler velocity measurements of SN 1986G, SN 1991bg, SN 2007on, SN 2011iv, and iPTF13ebh for ten different spectral line features. The uncertainty in the measurements is roughly $500 \mathrm{~km} \mathrm{~s}^{-1}$. Additionally, the average velocity values (and their associated $1 \sigma$ dispersion) of 


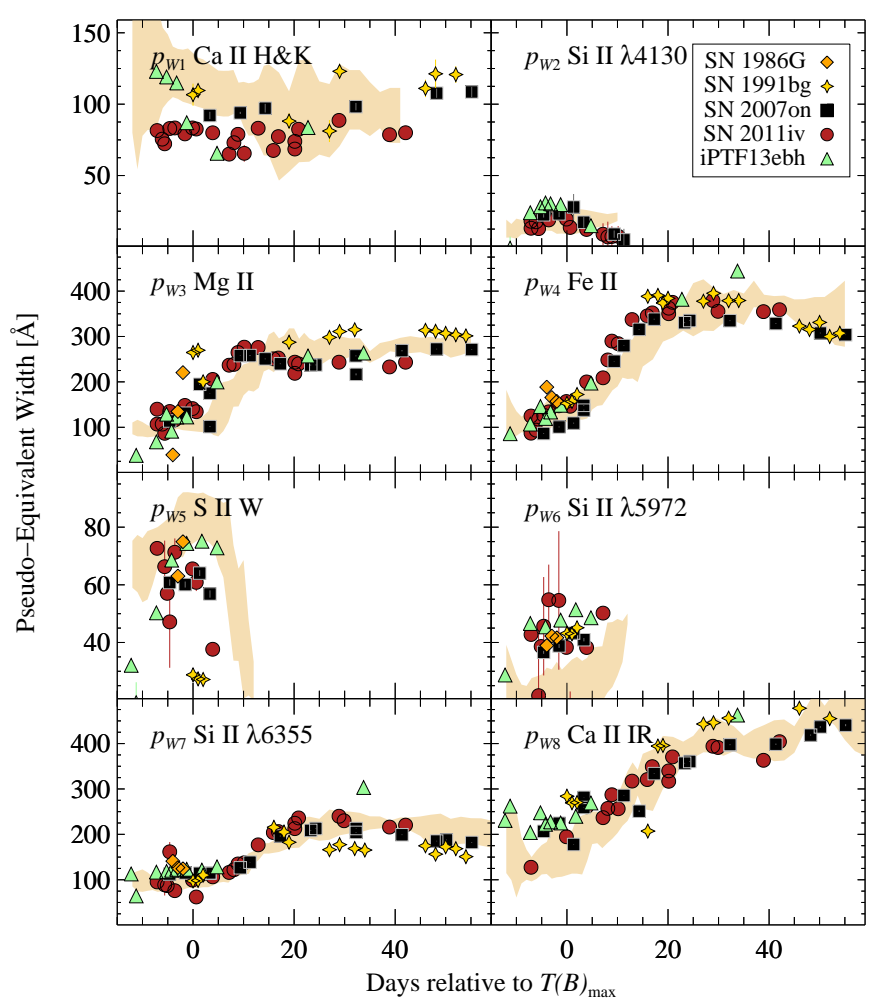

Fig. B.1. Temporal evolution of eight pEW spectral indicators. Black squares and red dots correspond to the pEW values of SN 2007on and SN 2011iv, respectively. Additionally, presented are pEW values determined from spectra of the subluminous Type Ia SN 1986G (orange diamond), SN 1991bg (yellow star), and the transitional SN Ia iPTF13ebh (Hsiao et al. 2015, green triangles). The yellow shaded areas corresponds to the average $\mathrm{pEW}$ values measured from a large set of normal SNe Ia (Folatelli et al. 2013).

seven absorption features obtained from the CSP-I SN Ia sample (Folatelli et al. 2013) are shown in Fig. B.2. Overall, the velocities of the various spectral features in the fast-declining SNe Ia are found to be marginally less than those of normal SNe Ia. Comparison of velocities and their temporal evolution among the fast-declining SNe Ia indicated no significant differences.

\section{Appendix C: Nuclear burning fronts in multi-dimensional objects}

The findings presented in Sect. 6.2 are based on comparisons of the light-curve properties of SN 2007on and SN 2011iv to a suite of spherical 1D DD explosion models though the physics and many aspects of nuclear burning fronts are inherently multidimentional be it the deflagration phase, the transition between deflagration to detonation, and the detonation phase.

During the last decades, significant progress has been made toward a better understanding of the physics of deflagration hydrodynamical flames in SNe Ia. On a microscopic scale, a deflagration propagates due to heat conduction by electrons. Though the laminar flame speed is well known, the fronts are hydrodymamical unstable which requires multi-dimensional simulations adopting various sub-grid scale model (Khokhlov 1995, 2000; Niemeyer \& Hillebrandt 1995b; Reinecke et al. 1999; Lisewski et al. 2000; Röpke et al. 2006; Gamezo et al. 2003, 2005a; Fink et al. 2014; Zel'Dovich et al. 1970). All groups found that Rayleigh-Taylor instabilities (RT) govern the morphology of the fronts in the regime of linear instabilities, and

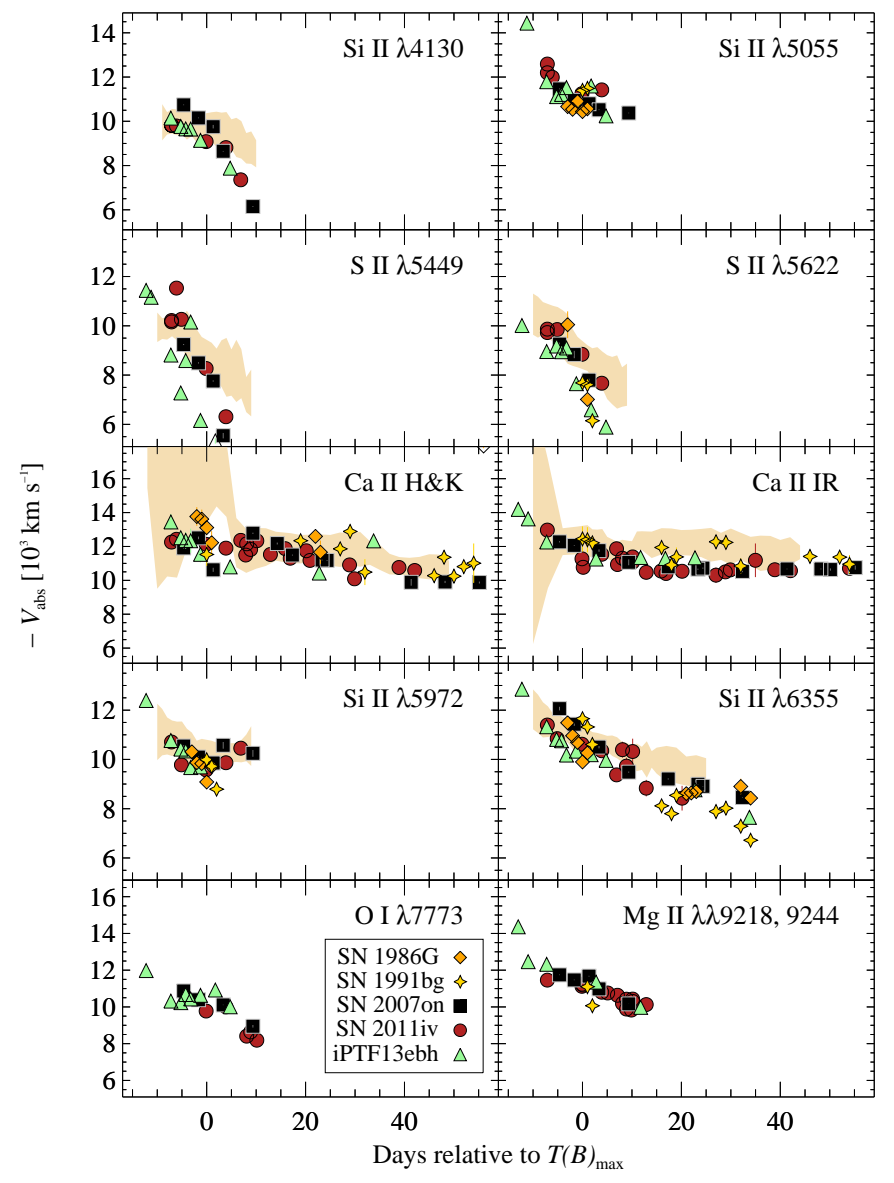

Fig. B.2. Temporal evolution of the Doppler velocity of prominent ions. Velocity measurements of SN 2007on (black squares) and SN 2011iv (red circles) are compared to measurements of SN 1986G, SN 1991bg, and iPTF 13ebh (colour coded as in Fig. B.1). The yellow shaded areas correspond to the average velocities measured from a large set of normal SNe Ia (Folatelli et al. 2013).

other instabilities (e.g. Kelvin-Helmholtz) become important. Differences in the simulations occur due to the adoption of various sub-grid models and due the sensitivity with respect to the initial conditions of the WD at the time of the thermonuclear runaway and multi-spot ignitions (Niemeyer et al. 1996; Calder et al. 2004; Plewa et al. 2004; Livne et al. 2005). All simulations to date predict significant large-scale mixing of burned and unburned material in the WD already during the deflagration phase.

Detailed studies of well-observed SNe Ia suggest there is a process at work which partially suppresses RT instabilities during the deflagration burning phase. For example, direct imaging of the supernova remnant (SNR) S Andromedae suggests a large Ca-free core, indicative of high-density burning and limited mixing (Fesen et al. 2007, 2015, 2016). Additional lines of evidence of partially suppressed RT mixing are significantly degraded spectral fits by models having an injection of radioactive material into the Si or S layers (Hoeflich et al. 2002), pot-bellied nebular line profiles (Höflich et al. 2004; Motohara et al. 2006; Maeda et al. 2010; Stritzinger et al. 2015; Diamond et al. 2015), and broad [Co III], [Fe II], and [Ar II] spectral features seen in late-time MIR spectra, which at the same time exhibit narrow ${ }^{58} \mathrm{Ni}$ lines (Gerardy et al. 2004; Telesco et al. 2015; Fesen et al. 2015). The underlying physics driving the suppression of RT mixing is currently unknown, though effects associated with 

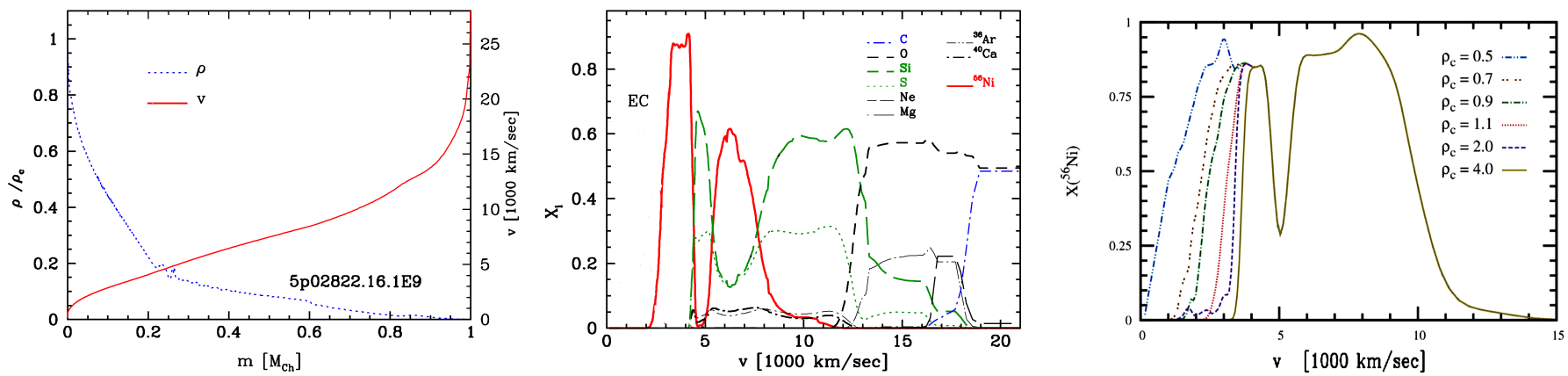

Fig. D.1. Simulation results corresponding to model 5p0z22.16.1E9 which best-fits the observations of SN 2007on. This DD model simulates the disruption of a WD that explodes with $\rho_{\mathrm{tr}}=1.6 \times 10^{7} \mathrm{~g} \mathrm{~cm}^{-3}$ and $\rho_{\mathrm{c}}=1.0 \times 10^{9} \mathrm{~g} \mathrm{~cm}^{-3}$. Left panel: both the density (blue dotted line) and velocity structures (red solid line) are shown vs. mass. Middle panel: element abundances of the most important elements out of 218 isotopes presented in mass fraction $\left(X_{i}\right)$ vs. velocity. The composition of the central region is dominated by numerous iron-group electron capture (EC) isotopes (Hoeflich et al. 1998; Brachwitz et al. 2000). Right panel: variation in the ${ }^{56} \mathrm{Ni}$ distribution vs. velocity with $\rho_{\mathrm{c}}$ varied from $(0.5-4.0) \times$ $10^{9} \mathrm{~g} \mathrm{~cm}^{-3}$ for a normal-bright SN Ia model (see Diamond et al. 2015).

the presence of prevalent magnetic fields could be a possibility which can suppress large scale instabilties (Höflich et al. 2004; Penney \& Hoeflich 2014; Remming \& Khokhlov 2014; Diamond et al. 2015; Hristov et al. 2016), or it may be related to the initial condition of the WD such as rapid rotation (Uenishi et al. 2003; Yoon \& Langer 2004; Hoeflich 2006). In this study, we adopt spherical explosion models as they naturally partially suppress RT mixing during the deflagration burning phase (Domínguez et al. 2001).

Hydrodynamical instabilities and the interplay between hydrodynamics and nuclear burning also plays a role in the transition from the deflagration to detonation burning phases. However, here the processes at play are not associated with large scale hydrodynamical instabilities, but rather to small scale mixing of burned and unburned material at the interface of a compressional shock wave following the so-called Zel'dovich mechanism (e.g. Niemeyer \& Hillebrandt 1995a; Khokhlov et al. 1997). Other mechanisms suggested invoke crossing shock waves produced in the highly turbulent medium (Livne 1999) and mixing by shear flows in rapidly, differentially rotating WDs (?Uenishi et al. 2003; Yoon et al. 2004; Yoon \& Langer 2004). The mechanism(s) for small scale mixing is still under debate and in all simulations the transition from the deflagration to detonation phase is initiated by microscopic mixing within the adopted parameterisation (see, e.g. Livne \& Arnett 1995; Niemeyer \& Hillebrandt 1995a; Khokhlov et al. 1997; Gamezo et al. 2005b; Ciaraldi-Schoolmann et al. 2013).

\section{Appendix D: Model comparisons}

We now present a comparison between predicted observables of the models and SNe 2007on and 2011iv. As a baseline we use models originating from a $5 M_{\odot}$ main sequence star with solar metallicity (Hoeflich et al. 2002). The best-fit model parameters are determined from the interpolations of an extend grid of DD models with $\rho_{\mathrm{c}}=0.5 \ldots 6 \times 10^{9} \mathrm{~g} \mathrm{~cm}^{-3}$ and $\rho_{\text {tr }}$ $=0.5 \ldots 2.7 \times 10^{7} \mathrm{~g} \mathrm{~cm}^{-3}$ using the $\Delta m_{15}-$ CMAGIC optimization for determining the model parameters (Hoeflich et al. 2017).

Comparison of key observables to predictions of these models (computed using the CSP-I passbands) are determined using the absolute $B$ - and $V$-band light curves, the $B-V$ colours and the $B$ - and $V$-band decline-rate parameters. We note that the $B-V$ colour offset noted in Sect. 3.2 is used to calculate the relative difference in the $\rho_{\mathrm{c}}$ between SNe 2007on and 2011iv in- dicates a factor of two differences in $\rho_{\mathrm{c}}$, assuming the conical value of $\rho_{\mathrm{c}}=2.0 \times 10^{9} \mathrm{~g} \mathrm{~cm}^{-3}$ for SN 2011iv.

The best-fit model for SN 2007on is characterised by $\rho_{\mathrm{c}}$ $=1.0 \times 10^{9} \mathrm{~g} \mathrm{~cm}^{-3}$ and $\rho_{\mathrm{tr}}=1.6 \times 10^{7} \mathrm{~g} \mathrm{~cm}^{-3}$ and corresponds to model 5p0z22.16.1E9 (see Hoeflich et al. 2017). Model 5p0z22.16.1E9 produces $0.008 M_{\odot}$ of stable ${ }^{58} \mathrm{Ni}$ and $0.32 M_{\odot}$ of radioactive ${ }^{56} \mathrm{Ni}$ mass of which $0.13 M_{\odot}$ is produced during the deflagration burning phase and $0.19 M_{\odot}$ during the detonation burning phase. The difference between the model observables $\left[M_{B}, B-V\right.$ at $\left.T(B)_{\max }, \Delta m_{15}(B), \Delta m_{15}(V)\right]$ of [-18.29 mag, $0.11 \mathrm{mag}, 1.69 \mathrm{mag}, 1.16 \mathrm{mag}]$ and those corresponding to SN 2007on of [ $-18.23 \pm 0.08 \mathrm{mag}, 0.08 \pm 0.01 \mathrm{mag}$, $1.96 \pm 0.01 \mathrm{mag}, 1.14 \pm 0.01 \mathrm{mag}]$ are $[-0.06 \mathrm{mag}, 0.03 \mathrm{mag}$, $-0.27 \mathrm{mag}, 0.02 \mathrm{mag}$ ], respectively.

The best-fit model for SN 2011iv is characterised by $\rho_{\mathrm{c}}$ $=2.0 \times 10^{9} \mathrm{~g} \mathrm{~cm}^{-3}$ and $\rho_{\mathrm{tr}}=1.7 \times 10^{7} \mathrm{~g} \mathrm{~cm}^{-3}$ and corresponds to model $5 \mathrm{p} 0 \mathrm{z} 22.18 \mathrm{~b}$, which is an interpolation between models 5p0z22.16 and 5p0z22.18 (Hoeflich et al. 2002). Model 5p0z22.18b produces $0.02 M_{\odot}$ of stable ${ }^{58} \mathrm{Ni}$ and $0.37 M_{\odot}$ of radioactive ${ }^{56} \mathrm{Ni}$ of which $0.16 M_{\odot}$ was produced during the deflagration burning phase and $0.21 M_{\odot}$ during the detonation burning phase. The difference between the model observables $\left[M_{B}, B-V\right.$ at $\left.T(B)_{\max }, \Delta m_{15}(B), \Delta m_{15}(V)\right]$ of [-18.64 mag, $0.09 \mathrm{mag}, 1.67 \mathrm{mag}, 1.08 \mathrm{mag}]$ and those corresponding to SN $2011 \mathrm{iv}$ of $[-18.83 \pm 0.08 \mathrm{mag}, 0.01 \pm 0.01 \mathrm{mag}, 1.77 \pm$ $0.01 \mathrm{mag}, 1.08 \pm 0.01 \mathrm{mag}]$ are $[-0.19 \mathrm{mag},+0.08 \mathrm{mag}$, $-0.10 \mathrm{mag}, 0.0 \mathrm{mag}]$, respectively. We note, as demonstrated in Fig. 11, that the UVOIR light curve computed for $5 \mathrm{p} 0 \mathrm{z} 22.18 \mathrm{~b}$ is found to fit the UVOIR light curve SN 2011iv fairly well.

As an example of the model calculations we show in Fig. D.1 the best-fit model for SN 2007on (i.e. 5p0z22.16.1E9) its density and velocity structures as a function of mass (left panel), its final abundance tomography (middle panel), and the effect of varying $\rho_{\mathrm{c}}$ on the final distribution of ${ }^{56} \mathrm{Ni}$ (right panel). The middle panel of Fig. D.1 demonstrates that the abundance in the inner region is dominated by electron capture elements, and the size of this region is determined by $\rho_{\mathrm{c}}$ (see right panel). We note that the outer abundance tomography is largely determined by the detonation burning phase and therefore on $\rho_{\text {tr }}$ (Diamond et al. 2015). This justifies the procedure to interpolate in $\rho_{\mathrm{c}}$ and $\rho_{\mathrm{tr}}$.

The interpolated best-fit model for SN 2011iv (i.e. model 5p0z22.18b) has an overall similar structure as that shown in Fig. D.1 for model 5p0z22.16.1E9, but its intermediate mass elements are shifted to a higher velocity by $\approx 1000 \mathrm{~km} \mathrm{~s}^{-1}$ and 


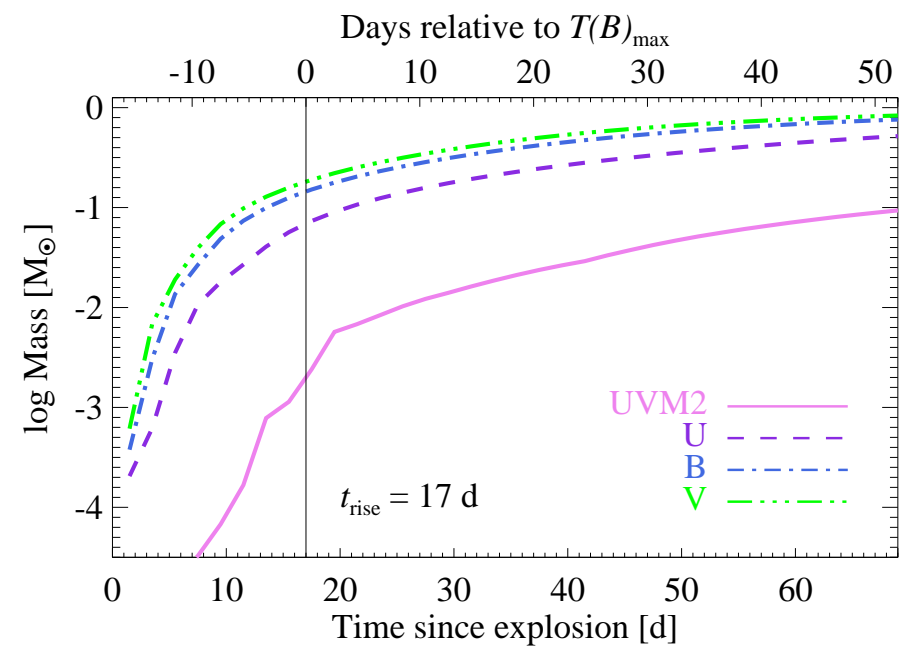

Fig. E.1. The amount of SN Ia ejecta mass probed by the emission contained within the uvm2, $U, B$, and $V$ bands as a function of days past explosion. These calculations are based on the DD model 5p0z22.18b. The masses are calculated by first averaging the opacity across the filter range, and then the total mass at this opacity is integrated from infinity down to an optical depth of unity.

the electron-capture elements extend to $3200 \mathrm{~km} \mathrm{~s}^{-1}$ (see right panel).

\section{Appendix E: Lack of evidence for CSM}

As shown in Fig. E.1, in the case of the DD model 5p0z22.18b the UV spectrum is formed in the outermost layers of the ejecta. By $+10 \mathrm{~d}$ past explosion the model indicates the Swift uvm 2 band samples a mass range $\sim 10^{-5}-10^{-2} M_{\odot}$, while the $U, B$, and $V$ bands sample the mass range $\sim 10^{-2}-0.7 M_{\odot}$. It follows that the early colours of SN 2007on and SN 2011iv (see Figs. 8 and 9) are driven by material from the outer ejecta. Since the colours of the two objects are very similar at peak, this suggests that the structure and condition of their outer layers are also similar.

The peak $B-V$ colours of $\mathrm{SN} 2007$ on $(0.08 \mathrm{mag})$ and SN 2011 iv (0.01 mag) are very similar and entirely consistent with the predictions between the DD models 5p0z22.16 and $5 \mathrm{p} 0 \mathrm{z} 22.18 \mathrm{~b}$, and they suggest no interaction between the SN ejecta and their environment. However, UV observations offer the opportunity to place limits on interaction expected to be produced when the rapidly expanding SN ejecta shocks CSM (Gerardy et al. 2004; Fesen et al. 2007).
To ascertain the velocity of the material associated with the outermost layers of the ejecta, we examine the UV pW01 feature (see Fig. E.2), which is located at the expected wavelength interval of a prevalent magnesium feature associated with material in the outer ejecta. As magnesium is a product of carbon burning, its presence is expected to be more dominant in subluminous SNe Ia since they experience incomplete burning compared to normal SNe Ia. Assuming the pW01 feature observed in SN 2011iv is dominated by absorption from the Mg II $\lambda \lambda 2796$, 2803 doublet resonance transition, we show in the left panel of Fig. E.2 its corresponding Doppler velocity at maximum absorption $\left(-V_{\mathrm{abs}}\right)$, while the right panel shows the evolution of the blue-wing velocity. Also included for comparison are identical measurements made for those SNe Ia with published UV spectra: SN 1992A (Kirshner et al. 1993), SN 2011fe (Mazzali et al. 2014), and SN 2013dy (Pan et al. 2015).

At maximum brightness, the $-V_{\text {abs }}$ of $\mathrm{Mg}$ II in SN 2011iv is in excess of $\sim 16000 \mathrm{~km} \mathrm{~s}^{-1}$ and decreases down to $\sim 14000 \mathrm{~km} \mathrm{~s}^{-1}$ within a month, while the highest velocity reached as inferred from the blue wing is $\sim 25000 \mathrm{~km} \mathrm{~s}^{-1}$. Interestingly, SN 2011fe exhibits $-V_{\text {abs }}$ and blue-wing velocity values that are consistent with SN 2011iv extending from maximum light to a month later, while those of SN 1992A and SN 2013dy are significantly higher, exhibiting $-V_{\text {abs }}$ and bluewing velocity values at maximum in excess of $20000 \mathrm{~km} \mathrm{~s}^{-1}$ and $\sim 27000 \mathrm{~km} \mathrm{~s}^{-1}$, respectively. This could be due to SN $2011 \mathrm{fe}$ having experienced enhanced mixing of carbon-burning products into the photospheric region (Nugent et al. 2011), thus leading to a reduction of carbon-burning products at high velocity and enhanced carbon-burning products at lower velocities.

In the $5 \mathrm{p} 0 \mathrm{z} 22.18 \mathrm{~b}$ model considered here, magnesium forms in a shell of $0.2 M_{\odot}$ with a velocity extent of $\sim 13250$ $19350 \mathrm{~km} \mathrm{~s}^{-1}$. This is consistent with the velocity measurements of SN 2011 iv. For comparison, in the normal SN Ia model 5 p0z22.25, the magnesium forms in a shell of $0.1 M_{\odot}$, extending from between $\sim 15500$ to $>25000 \mathrm{~km} \mathrm{~s}^{-1}$. In the case of the $5 \mathrm{p} 0 \mathrm{z} 22.18 \mathrm{~b}$ model, the outer unburned $\mathrm{C}+\mathrm{O}$ mass amounts to $0.04 M_{\odot}$. This implies no significant pulsation or interaction occurred with material in excess of $0.04 M_{\odot}$ during the explosion. For example, if another $0.04 M_{\odot}$ of material would interact with the peak blue-wing velocity, we would expect (owing to momentum conservation) to reduce the peak blue-wing velocity down to $16000 \mathrm{~km} \mathrm{~s}^{-1}$.

Finally, we note that the strength of the pW02 feature, which is probably produced by a multitude of Fe II lines, is determined by the primordial abundances of Fe-group elements at high velocity. We therefore expect this feature to be similar among the $\mathrm{SNe}$, as demonstrated in the bottom panel of Fig. 13. 
C. Gall et al.: CSP observations of SN 2007on and SN 2011iv
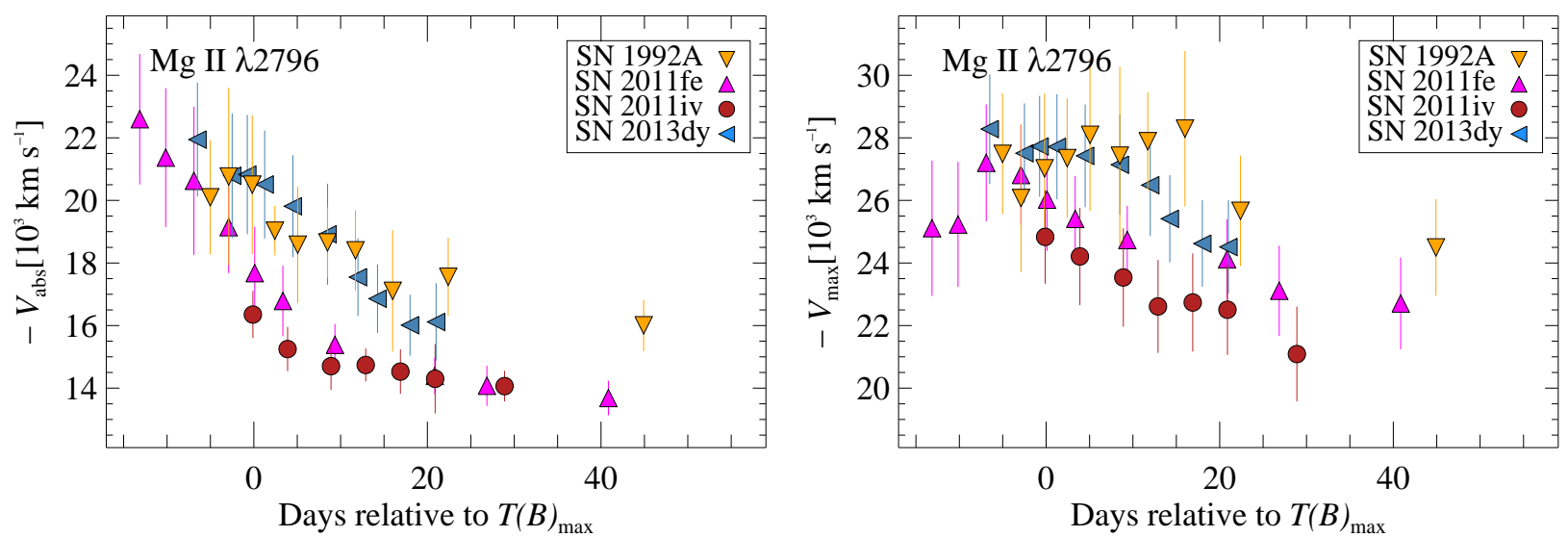

Fig. E.2. Left: evolution of the Doppler velocity at maximum absorption evaluated for the pW01 feature (see Fig. 13) attributed to the Mg II $\lambda \lambda 2796,2803$ doublet for SN 1992A, SN 2011fe, SN $2011 \mathrm{iv}$, and SN 2013dy. Right: blue-wing velocity measurements of the Mg II feature for the same objects. 


\section{Appendix F: Additional tables}

Table F.1. UVOT UV photometry of SN 2007on and SN 2011iv.

\begin{tabular}{|c|c|c|c|c|}
\hline MJD & $\overline{\text { Phase }^{a}}$ & $u v w 2$ & uvm2 & uvw1 \\
\hline \multicolumn{5}{|c|}{ SN 2007on } \\
\hline 54410.7 & -9.1 & $16.524(080)$ & 16.783(067) & $15.170(065)$ \\
\hline 54412.1 & -7.9 & $16.068(075)$ & $16.196(067)$ & $14.631(050)$ \\
\hline 54414.4 & -5.4 & $15.598(065)$ & $15.804(066)$ & $14.237(045)$ \\
\hline 54416.5 & -3.3 & $15.574(067)$ & $15.785(066)$ & $14.205(045)$ \\
\hline 54418.9 & -1.0 & $15.786(075)$ & $16.005(067)$ & $14.404(046)$ \\
\hline 54420.5 & +0.7 & $15.975(075)$ & $16.094(068)$ & $14.594(050)$ \\
\hline 54422.5 & +2.7 & $16.220(076)$ & $16.472(070)$ & $14.912(055)$ \\
\hline 54423.9 & +4.1 & $16.321(094)$ & $16.578(114)$ & $15.150(077)$ \\
\hline 54424.7 & +4.9 & $16.526(077)$ & $16.725(072)$ & $15.242(063)$ \\
\hline 54426.3 & +6.5 & $16.781(077)$ & $16.991(073)$ & $15.532(064)$ \\
\hline 54428.7 & +8.9 & $17.052(079)$ & $17.176(078)$ & $15.841(065)$ \\
\hline 54430.4 & +10.6 & $17.331(080)$ & $17.288(080)$ & $16.074(066)$ \\
\hline 54432.5 & +12.7 & $17.502(081)$ & $17.547(082)$ & $16.314(067)$ \\
\hline 54435.5 & +15.7 & $17.654(081)$ & $17.629(080)$ & $16.481(067)$ \\
\hline 54436.8 & +17.0 & $17.704(082)$ & $17.703(082)$ & $16.528(067)$ \\
\hline 54438.8 & +19.0 & $17.794(085)$ & $17.769(088)$ & $16.610(070)$ \\
\hline 54442.8 & +23.0 & $17.837(083)$ & $17.809(083)$ & $16.801(069)$ \\
\hline 54446.1 & +26.3 & $18.023(088)$ & 17.957(092) & $16.973(074)$ \\
\hline 54450.1 & +30.3 & 18.101(088) & $17.969(089)$ & $17.072(073)$ \\
\hline 54454.2 & +34.4 & $18.165(090)$ & $18.084(097)$ & $17.227(076)$ \\
\hline 54458.8 & +39.0 & $18.389(093)$ & $18.236(098)$ & $17.350(077)$ \\
\hline 54461.3 & +41.5 & $18.447(089)$ & $18.353(094)$ & $17.512(075)$ \\
\hline 54468.7 & +48.9 & $18.746(095)$ & $18.850(110)$ & $17.719(079)$ \\
\hline \multicolumn{5}{|c|}{ SN 2011iv } \\
\hline 55900.2 & -5.9 & & $\cdots$ & $13.922(046)$ \\
\hline 55900.3 & -5.8 & $15.386(079)$ & $\ldots$ & $\ldots$ \\
\hline 55901.4 & -4.7 & $15.182(064)$ & $\ldots$ & $13.820(047)$ \\
\hline 55901.8 & -4.3 & $15.163(068)$ & $\ldots$ & $13.820(048)$ \\
\hline 55901.9 & -4.2 & $15.199(080)$ & $\ldots$ & $\ldots$ \\
\hline 55902.5 & -3.6 & $15.143(069)$ & $15.605(068)$ & $\ldots$ \\
\hline 55902.6 & -3.5 & $\ldots$ & $\ldots$ & $13.798(046)$ \\
\hline 55903.3 & -2.8 & $15.160(065)$ & $\ldots$ & $\ldots$ \\
\hline 55903.4 & -2.7 & $\ldots$ & $15.560(066)$ & $13.811(048)$ \\
\hline 55904.8 & -1.3 & $\ldots$ & $\ldots$ & $13.890(048)$ \\
\hline 55905.7 & -0.4 & $15.357(082)$ & $\ldots$ & $\ldots$ \\
\hline 55906.6 & +0.5 & $15.322(067)$ & $15.720(067)$ & $14.049(048)$ \\
\hline 55907.5 & +1.4 & $15.485(079)$ & $\ldots$ & $\ldots$ \\
\hline 55909.3 & +3.2 & $15.708(079)$ & $16.059(070)$ & $14.390(055)$ \\
\hline 55911.5 & +5.4 & $16.092(083)$ & $16.486(074)$ & $14.849(067)$ \\
\hline 55913.3 & +7.2 & $16.418(088)$ & $16.845(082)$ & $\ldots$ \\
\hline 55913.5 & +7.4 & $\ldots$ & $\ldots$ & $15.147(072)$ \\
\hline 55915.7 & +9.6 & $16.827(096)$ & $17.277(090)$ & $15.525(075)$ \\
\hline 55917.9 & +11.8 & $17.197(106)$ & 17.631(101) & $16.014(086)$ \\
\hline 55919.8 & +13.7 & $17.437(116)$ & 17.911(114) & $16.308(094)$ \\
\hline 55922.7 & +16.6 & $17.788(133)$ & $18.063(121)$ & $16.545(101)$ \\
\hline 55925.5 & +19.4 & $17.934(140)$ & $18.368(139)$ & $16.806(114)$ \\
\hline 55928.7 & +22.6 & $18.060(151)$ & $18.368(141)$ & $17.087(140)$ \\
\hline 55931.6 & +25.5 & $18.356(176)$ & $18.480(149)$ & $17.109(140)$ \\
\hline 55934.2 & +28.1 & $18.452(184)$ & $18.449(145)$ & $17.139(131)$ \\
\hline
\end{tabular}

Notes. $1 \sigma$ uncertainties given in parentheses are in millimag. ${ }^{(a)}$ Days relative to $T(B)_{\max }$. 
C. Gall et al.: CSP observations of SN 2007on and SN 2011iv

Table F.2. Optical photometry of the local sequence stars in the field of NGC 1404 in the "standard" system.

\begin{tabular}{|c|c|c|c|c|c|c|c|c|}
\hline Star & $\alpha(2000)$ & $\delta(2000)$ & $u^{\prime}$ & $g^{\prime}$ & $r^{\prime}$ & $i^{\prime}$ & $B$ & V \\
\hline \multicolumn{9}{|c|}{ SN 2007on } \\
\hline 1 & 03:38:37.30 & $-35: 33: 58.6$ & $16.400(021)$ & $14.266(005)$ & $13.477(005)$ & $13.195(006)$ & $14.866(005)$ & $13.907(004)$ \\
\hline 2 & 03:38:41.79 & $-35: 35: 39.2$ & $15.778(015)$ & $14.261(005)$ & 13.681(005) & $13.510(006)$ & $14.718(005)$ & 13.996(004) \\
\hline 3 & 03:38:49.88 & $-35: 32: 14.1$ & $15.533(014)$ & $14.288(005)$ & $13.869(005)$ & $13.764(006)$ & $14.664(005)$ & $14.084(004)$ \\
\hline 4 & 03:39:07.63 & $-35: 32: 46.5$ & $15.619(015)$ & $14.552(005)$ & $14.179(005)$ & $14.090(006)$ & $14.903(005)$ & $14.373(004)$ \\
\hline 5 & 03:39:08.62 & $-35: 32: 11.7$ & $16.179(019)$ & $14.693(005)$ & $14.202(005)$ & $14.082(006)$ & $15.118(006)$ & $14.463(004)$ \\
\hline 6 & $03: 38: 57.88$ & $-35: 34: 08.2$ & $17.093(035)$ & $15.945(006)$ & $15.514(006)$ & $15.398(007)$ & $16.319(009)$ & 15.733(006) \\
\hline 7 & 03:39:08.80 & $-35: 33: 18.9$ & $17.212(041)$ & $16.198(007)$ & $15.834(006)$ & $15.733(008)$ & $16.535(010)$ & $16.028(007)$ \\
\hline 8 & 03:39:06.75 & $-35: 34: 48.0$ & $\ldots$ & $17.510(014)$ & $16.419(008)$ & $15.994(009)$ & 18.103(033) & $17.046(013)$ \\
\hline 9 & 03:38:37.33 & $-35: 35: 12.4$ & $\cdots$ & $18.255(025)$ & $16.911(011)$ & $15.538(007)$ & $18.865(084)$ & $17.658(022)$ \\
\hline 10 & 03:39:08.98 & $-35: 33: 00.0$ & $\ldots$ & $18.084(021)$ & $17.305(014)$ & $16.978(016)$ & $18.581(057)$ & $17.744(023)$ \\
\hline 11 & 03:38:37.45 & $-35: 35: 52.3$ & $\cdots$ & 18.011(019) & $17.369(015)$ & $17.186(020)$ & $18.336(044)$ & $17.675(022)$ \\
\hline 13 & 03:38:48.09 & $-35: 32: 49.5$ & $\ldots$ & $19.510(084)$ & $18.684(048)$ & $18.291(057)$ & $\ldots$ & $\ldots$ \\
\hline 14 & 03:38:41.83 & $-35: 36: 49.1$ & $\cdots$ & $\ldots$ & $18.699(051)$ & $17.751(032)$ & $\ldots$ & $\cdots$ \\
\hline 15 & 03:38:51.21 & $-35: 31: 20.5$ & $\cdots$ & $19.436(104)$ & $18.267(033)$ & $17.172(020)$ & 18.841(199) & $19.215(199)$ \\
\hline 16 & 03:39:04.84 & $-35: 36: 40.0$ & $\cdots$ & $19.606(115)$ & $18.623(045)$ & $18.226(055)$ & $\ldots$ & $\ldots$ \\
\hline 17 & 03:39:06.74 & $-35: 32: 10.8$ & $\ldots$ & $19.590(165)$ & $18.525(043)$ & $17.358(023)$ & $\ldots$ & $\ldots$ \\
\hline 18 & 03:38:40.93 & $-35: 32: 06.9$ & $\ldots$ & $\ldots$ & $19.081(082)$ & $17.683(031)$ & $\ldots$ & $\ldots$ \\
\hline 19 & 03:38:40.66 & $-35: 36: 54.0$ & $\ldots$ & $19.693(179)$ & $19.257(089)$ & $18.651(151)$ & $\cdots$ & $\ldots$ \\
\hline 21 & 03:38:33.98 & $-35: 35: 38.5$ & $\ldots$ & $19.580(110)$ & $19.123(101)$ & 18.591(193) & $\ldots$ & $\ldots$ \\
\hline \multicolumn{9}{|c|}{ SN 2011iv } \\
\hline 1 & $03: 38: 37.30$ & $-35: 33: 58.6$ & $16.425(062)$ & $14.258(033)$ & $13.457(008)$ & $13.142(006)$ & 14.864(016) & $13.882(007)$ \\
\hline 2 & 03:38:41.79 & $-35: 35: 39.2$ & $15.762(022)$ & $14.256(032)$ & $13.669(008)$ & $13.462(007)$ & $14.710(015)$ & 13.984(011) \\
\hline 3 & 03:38:49.88 & $-35: 32: 14.1$ & $15.491(027)$ & $14.281(028)$ & $13.855(010)$ & $13.707(008)$ & $14.655(020)$ & $14.068(013)$ \\
\hline 4 & 03:39:07.63 & $-35: 32: 46.5$ & $15.621(025)$ & $14.550(031)$ & $14.168(011)$ & $14.032(012)$ & 14.891(017) & $14.365(013)$ \\
\hline 5 & 03:39:08.62 & $-35: 32: 11.7$ & $16.165(039)$ & $14.687(030)$ & $14.195(010)$ & $14.036(013)$ & $15.116(021)$ & $14.453(015)$ \\
\hline 6 & $03: 38: 57.88$ & $-35: 34: 08.2$ & $17.137(048)$ & $15.937(032)$ & $15.499(018)$ & $15.355(024)$ & $16.331(028)$ & $15.720(023)$ \\
\hline 7 & 03:39:08.79 & $-35: 33: 18.9$ & $17.289(039)$ & $16.184(052)$ & $15.817(024)$ & $15.683(030)$ & $16.520(029)$ & $16.007(030)$ \\
\hline 8 & 03:39:06.75 & $-35: 34: 48.0$ & $\ldots$ & $17.524(054)$ & $16.399(043)$ & $15.960(030)$ & $18.215(035)$ & $17.058(042)$ \\
\hline 9 & 03:38:37.33 & $-35: 35: 12.4$ & $\cdots$ & $18.244(045)$ & $16.907(026)$ & $15.498(029)$ & $19.229(181)$ & $17.676(042)$ \\
\hline 10 & 03:39:08.98 & $-35: 33: 00.0$ & $\ldots$ & $18.070(039)$ & $17.272(070)$ & $16.989(058)$ & $18.576(089)$ & $17.714(041)$ \\
\hline 11 & 03:38:37.45 & $-35: 35: 52.3$ & $\ldots$ & $17.972(047)$ & $17.355(073)$ & $17.138(061)$ & $18.457(038)$ & $17.691(062)$ \\
\hline
\end{tabular}


A\&A 611, A58 (2018)

Table F.3. Near-IR photometry of the local sequence stars in the field of NGC 1404 in the "natural" system.

\begin{tabular}{|c|c|c|c|c|c|}
\hline Star & $\alpha(2000)$ & $\delta(2000)$ & $Y$ & $J$ & $H$ \\
\hline \multicolumn{6}{|c|}{ SN 2007on } \\
\hline 101 & 03:38:41.79 & $-35: 35: 39.0$ & $12.779(015)$ & $12.485(028)$ & $12.041(013)$ \\
\hline 102 & $03: 39: 14.28$ & $-35: 31: 43.0$ & $12.794(011)$ & $12.565(020)$ & $12.291(011)$ \\
\hline 103 & 03:39:19.77 & $-35: 31: 55.7$ & $14.276(010)$ & $13.986(023)$ & $13.505(019)$ \\
\hline 104 & 03:39:06.76 & $-35: 34: 47.9$ & $15.058(053)$ & $14.646(033)$ & $13.980(032)$ \\
\hline 105 & 03:38:51.10 & $-35: 31: 21.1$ & $15.865(042)$ & $15.399(033)$ & $14.847(023)$ \\
\hline 106 & 03:39:06.73 & $-35: 32: 11.0$ & $15.987(034)$ & $15.512(046)$ & $14.934(083)$ \\
\hline 107 & 03:39:17.84 & $-35: 36: 52.0$ & $16.112(023)$ & $15.626(044)$ & $14.905(063)$ \\
\hline 108 & 03:39:19.31 & $-35: 36: 31.3$ & $16.092(030)$ & $15.828(046)$ & $15.438(108)$ \\
\hline 109 & 03:38:49.81 & $-35: 37: 41.8$ & $16.767(056)$ & $16.320(118)$ & $15.697(093)$ \\
\hline 110 & 03:39:17.19 & $-35: 31: 39.2$ & $17.068(089)$ & $16.689(161)$ & $15.936(125)$ \\
\hline 111 & 03:39:04.86 & $-35: 36: 39.6$ & $17.296(167)$ & $16.858(078)$ & $16.192(129)$ \\
\hline 112 & 03:38:48.09 & $-35: 32: 49.6$ & & $17.169(107)$ & $\ldots$ \\
\hline 113 & 03:39:15.06 & $-35: 38: 18.0$ & $18.099(191)$ & $17.516(161)$ & $\ldots$ \\
\hline 114 & 03:39:13.60 & $-35: 37: 55.5$ & $17.853(106)$ & $\ldots$ & $16.914(089)$ \\
\hline 115 & 03:39:07.93 & $-35: 37: 46.8$ & $17.759(167)$ & $17.318(149)$ & $16.740(081)$ \\
\hline 116 & 03:39:17.60 & $-35: 36: 08.3$ & $18.130(029)$ & $\ldots$ & $16.949(133)$ \\
\hline 117 & 03:39:07.54 & $-35: 36: 58.9$ & $18.327(058)$ & $17.432(166)$ & $\ldots$ \\
\hline 118 & 03:38:57.88 & $-35: 34: 08.1$ & $14.823(025)$ & $14.580(040)$ & $14.238(046)$ \\
\hline 119 & 03:38:49.86 & $-35: 32: 14.6$ & $13.113(013)$ & $12.863(021)$ & $12.534(013)$ \\
\hline 120 & 03:38:50.42 & $-35: 31: 46.3$ & $\ldots$ & $17.036(146)$ & $\ldots$ \\
\hline 121 & 03:38:52.98 & $-35: 38: 08.2$ & $16.114(039)$ & $15.810(072)$ & $15.294(093)$ \\
\hline 122 & 03:39:16.09 & $-35: 37: 51.4$ & 11.487(013) & $11.247(027)$ & $10.947(025)$ \\
\hline 123 & 03:39:13.98 & $-35: 35: 58.6$ & $16.604(042)$ & $16.130(058)$ & $15.561(066)$ \\
\hline 124 & 03:39:08.78 & $-35: 33: 19.2$ & $15.124(025)$ & $14.867(018)$ & $14.520(034)$ \\
\hline 125 & 03:39:08.99 & $-35: 32: 59.9$ & $16.233(045)$ & $15.863(061)$ & $15.322(077)$ \\
\hline 126 & 03:39:07.63 & $-35: 32: 46.6$ & 13.466(018) & $13.223(026)$ & $12.902(019)$ \\
\hline 127 & 03:39:08.60 & $-35: 32: 11.8$ & $13.425(017)$ & 13.161(024) & $12.821(015)$ \\
\hline \multicolumn{6}{|c|}{ SN 2011iv } \\
\hline 101 & 03:38:55.09 & $-35: 36: 06.4$ & $11.349(088)$ & $11.192(127)$ & \\
\hline 102 & 03:38:50.15 & $-35: 35: 32.1$ & $15.076(128)$ & $14.696(070)$ & $14.112(086)$ \\
\hline 104 & 03:38:48.55 & $-35: 35: 31.2$ & $\ldots$ & $16.984(184)$ & \\
\hline
\end{tabular}


C. Gall et al.: CSP observations of SN 2007on and SN 2011iv

Table F.4. Optical photometry of SN 2007on and SN 2011iv in the "natural" system.

\begin{tabular}{|c|c|c|c|c|c|c|}
\hline JD-2 450000 & $u$ (mag) & $g$ (mag) & $r$ (mag) & $i$ (mag) & $B(\mathrm{mag})$ & $V(\mathrm{mag})$ \\
\hline \multicolumn{7}{|c|}{ SN 2007on } \\
\hline 4411.79 & $14.466(009)$ & & $14.176(008)$ & $14.330(010)$ & $14.174(007)$ & $14.173(006)$ \\
\hline 4413.82 & $13.971(017)$ & $13.584(016)$ & $13.633(016)$ & $13.805(017)$ & $13.655(016)$ & $13.650(016)$ \\
\hline 4417.72 & $13.652(009)$ & $13.085(006)$ & $\ldots$ & $13.368(013)$ & $\ldots$ & $13.128(006)$ \\
\hline 4418.75 & & 13.009(007) & $\ldots$ & $\ldots$ & $13.136(006)$ & $13.058(008)$ \\
\hline 4421.72 & $13.935(009)$ & ... & $\ldots$ & $\ldots$ & 13.095(004) & \\
\hline 4422.73 & $14.043(014)$ & $13.002(006)$ & $12.950(006)$ & $\ldots$ & $13.161(006)$ & $12.957(006)$ \\
\hline 4423.73 & $14.217(011)$ & $\ldots$ & $\ldots$ & $13.564(013)$ & $\ldots$ & $13.020(008)$ \\
\hline 4425.73 & $14.492(010)$ & $\ldots$ & $13.100(004)$ & & $\ldots$ & \\
\hline 4426.66 & $\ldots$ & $13.313(005)$ & 13.149(006) & $13.706(013)$ & $13.588(006)$ & $13.118(006)$ \\
\hline 4427.68 & $\ldots$ & $\ldots$ & $13.201(008)$ & $\ldots$ & $13.752(009)$ & $\ldots$ \\
\hline 28.72 & $15.102(024)$ & $\ldots$ & $\ldots$ & $\ldots$ & $13.948(006)$ & $13.164(009)$ \\
\hline 9.69 & $\ldots$ & $13.734(005)$ & $13.365(005)$ & $\ldots$ & 14.091(006) & $\ldots$ \\
\hline .67 & $15.498(023)$ & 13.833(006) & $\ldots$ & $13.737(013)$ & $14.278(006)$ & $\ldots$ \\
\hline & $16.207(C$ & $14.541(007)$ & $13.640(007)$ & $13.740(013)$ & $\ldots$ & $13.956(008)$ \\
\hline 4440.69 & $16.625(023)$ & & 14.094 & $\ldots$ & $\ldots$ & \\
\hline 4443.73 & & $15.269(005)$ & $14.411(0$ & $\cdots$ & $15.675(007)$ & $14.690(005)$ \\
\hline 4445.73 & $16.837(017)$ & $\ldots$ & $14.528(006)$ & $\ldots$ & $15.765(007)$ & $14.813(006)$ \\
\hline 4447.66 & $16.915(027)$ & $15.443(008)$ & & $14.673(012)$ & .. & $\ldots$ \\
\hline 4449.70 & $17.052(030)$ & $15.536(008)$ & $14.768(009)$ & & $15.931(009)$ & $14.986(009)$ \\
\hline 4453.64 & $17.119(033)$ & $\ldots$ & $14.963(007)$ & $15.005(011)$ & $16.067(009)$ & $15.154(008)$ \\
\hline 4455.62 & $17.209(036)$ & $\ldots$ & $15.052(008)$ & $15.109(011)$ & $16.107(009)$ & $15.230(007)$ \\
\hline 4456.67 & $17.225(041)$ & $\ldots$ & $15.097(006)$ & $15.147(011)$ & $\ldots$ & $\ldots$ \\
\hline 4457.63 & $17.236(028)$ & $15.766(008)$ & $15.132(007)$ & $15.203(011)$ & $16.180(010)$ & \\
\hline 4458.69 & $17.292(032)$ & $\ldots$ & $15.202(008)$ & $15.243(011)$ & $16.196(010)$ & $15.335(009)$ \\
\hline 4462.73 & $17.409(025)$ & $\ldots$ & & $15.411(011)$ & $16.311(009)$ & $15.465(007)$ \\
\hline 7.63 & 17.57 & $\ldots$ & $15.552(007)$ & $15.626(011)$ & $16.424(009)$ & $\ldots$ \\
\hline 8.64 & 17.65 & $\ldots$ & $15.587(008)$ & $15.665(013)$ & $16.450(010)$ & $15.651(011)$ \\
\hline 70.59 & $17.628(039)$ & $16.090(009)$ & $15.683(012)$ & $15.758(014)$ & $16.469(011)$ & $\ldots$ \\
\hline 4472.61 & $17.711(0$ & $\ldots$ & $\ldots$ & $15.822(0$ & $16.537(0$ & $\ldots$ \\
\hline 4475.61 & $17.791(0$ & $16.199(007)$ & $\ldots$ & $15.948(012)$ & 16.56 & $\cdots$ \\
\hline 4476.65 & $17.790(0$ & $\ldots$ & $15.917(010)$ & $\ldots$ & 16.608( & $\ldots$ \\
\hline 4484.59 & $18.048(078)$ & $\ldots$ & $\ldots$ & $16.272(014)$ & $16.720(013)$ & $16.103(010)$ \\
\hline 4488.58 & $18.219(086)$ & $\ldots$ & $16.399(010)$ & $\ldots$ & $16.844(014)$ & $16.231(010)$ \\
\hline 4490.58 & $18.232(030)$ & $16.485(007)$ & $16.477(009)$ & $\ldots$ & $16.869(010)$ & \\
\hline 4494.62 & $18.344(023)$ & $\ldots$ & $16.639(009)$ & $\ldots$ & $16.932(009)$ & $16.396(009)$ \\
\hline 4497.62 & $18.408(083)$ & $\ldots$ & $\ldots$ & $16.699(014)$ & $16.987(013)$ & $16.500(009)$ \\
\hline 4499.60 & $18.468(103)$ & $16.682(009)$ & $16.841(009)$ & $16.750(014)$ & $17.026(016)$ & $16.557(019)$ \\
\hline 4508.61 & $18.841(065)$ & $16.896(009)$ & $17.212(012)$ & $17.021(014)$ & $17.203(013)$ & $16.817(011)$ \\
\hline
\end{tabular}

Notes. Values in parentheses are $1 \sigma$ measurement uncertainties in millimag. 
Table F.4. continued.

\begin{tabular}{|c|c|c|c|c|c|c|}
\hline JD-2 450000 & $u$ (mag) & $g$ (mag) & $r(\mathrm{mag})$ & $i$ (mag) & $B$ (mag) & $V$ (mag) \\
\hline \multicolumn{7}{|c|}{ SN 2011iv } \\
\hline 5902.68 & $12.994(016)$ & $12.564(014)$ & $12.666(009)$ & $12.966(010)$ & $12.611(013)$ & $12.653(009)$ \\
\hline 5903.67 & $12.946(014)$ & 12.491(013) & $12.609(009)$ & 12.952(008) & $12.536(009)$ & $12.586(008)$ \\
\hline 5904.66 & $12.962(021)$ & 12.434(017) & $12.551(009)$ & $12.950(012)$ & $12.523(014)$ & $12.531(009)$ \\
\hline 5905.71 & 13.001(016) & $12.419(014)$ & $12.534(011)$ & $13.015(014)$ & $12.495(012)$ & $12.501(011)$ \\
\hline 5906.73 & $13.054(018)$ & $12.459(014)$ & $12.511(010)$ & $13.010(017)$ & $12.514(013)$ & $12.473(009)$ \\
\hline 5907.67 & $13.146(017)$ & $12.424(014)$ & $12.480(009)$ & $13.002(017)$ & $12.525(011)$ & $12.441(008)$ \\
\hline 5908.73 & $\ldots$ & $\ldots$ & $\ldots$ & $13.053(017)$ & $\ldots$ & $\ldots$ \\
\hline 5909.68 & $\ldots$ & $\ldots$ & $\ldots$ & $13.040(017)$ & $\cdots$ & $\cdots$ \\
\hline 5910.68 & $\cdots$ & $\ldots$ & $\ldots$ & $13.047(017)$ & $\cdots$ & $\ldots$ \\
\hline 5911.63 & $\ldots$ & $\ldots$ & $\ldots$ & 13.142(017) & $\ldots$ & $\ldots$ \\
\hline 5912.67 & $\ldots$ & $\ldots$ & $\ldots$ & $13.116(017)$ & $\ldots$ & $\ldots$ \\
\hline 5915.59 & $14.406(018)$ & $\cdots$ & $\ldots$ & $13.205(017)$ & $13.336(012)$ & $12.731(011)$ \\
\hline 5916.63 & $14.647(013)$ & $\ldots$ & $12.874(010)$ & $13.206(008)$ & $13.528(009)$ & $12.856(008)$ \\
\hline 5917.63 & $14.890(015)$ & $13.343(014)$ & 12.907(009) & 13.188(009) & $13.720(011)$ & $12.951(008)$ \\
\hline 5918.61 & 15.098(013) & 13.453(012) & $12.930(006)$ & 13.174(008) & 13.867(009) & $13.043(007)$ \\
\hline 5922.62 & 15.742(016) & $\ldots$ & $\ldots$ & $\ldots$ & $\ldots$ & $\ldots$ \\
\hline 5923.58 & $15.841(016)$ & $\ldots$ & $\ldots$ & $\ldots$ & $\ldots$ & $\ldots$ \\
\hline 5924.60 & $15.939(015)$ & & 13.197(012) & $13.268(016)$ & $14.669(013)$ & \\
\hline 5925.57 & $16.009(013)$ & $14.295(013)$ & $13.284(010)$ & $13.280(012)$ & $14.762(011)$ & $13.621(009)$ \\
\hline 5926.54 & $16.104(015)$ & $14.384(013)$ & $13.349(012)$ & $13.317(013)$ & $14.867(011)$ & $13.702(007)$ \\
\hline 5928.58 & $16.227(015)$ & $14.603(013)$ & $13.586(012)$ & $13.473(009)$ & $15.032(009)$ & $13.891(007)$ \\
\hline 5929.57 & $\ldots$ & $14.538(013)$ & $\ldots$ & $13.526(013)$ & $14.999(010)$ & $\ldots$ \\
\hline 5930.60 & $\ldots$ & 14.742(013) & $13.739(010)$ & $13.669(008)$ & $15.185(009)$ & $14.076(007)$ \\
\hline 5931.58 & $16.418(016)$ & $14.764(012)$ & 13.798(009) & 13.702(007) & $15.251(010)$ & 14.142(008) \\
\hline 5932.58 & $16.481(016)$ & $14.896(017)$ & 13.903(013) & 13.817(012) & $15.305(012)$ & $14.217(012)$ \\
\hline 5933.58 & $16.508(016)$ & 14.924(014) & $13.968(014)$ & $13.870(010)$ & $15.361(012)$ & $14.250(009)$ \\
\hline 5934.58 & $16.561(016)$ & $14.968(016)$ & $14.032(015)$ & $13.936(014)$ & $15.397(012)$ & $14.313(010)$ \\
\hline 5935.57 & $16.598(016)$ & 14.993(014) & $14.088(009)$ & $14.020(008)$ & $15.432(010)$ & $14.381(009)$ \\
\hline 5937.56 & $16.669(014)$ & 15.087(013) & $14.206(012)$ & $14.216(016)$ & $15.511(011)$ & $14.448(007)$ \\
\hline 5938.58 & 16.691(016) & $\ldots$ & $\ldots$ & 14.167(017) & $15.544(015)$ & $\ldots$ \\
\hline 5939.57 & $16.737(016)$ & $15.126(015)$ & $14.283(016)$ & $14.180(017)$ & $15.571(013)$ & $\ldots$ \\
\hline 5940.57 & $16.762(017)$ & $15.155(015)$ & $14.338(016)$ & 14.287(017) & $15.587(012)$ & $14.549(011)$ \\
\hline 5941.56 & $16.802(016)$ & $15.179(016)$ & $14.398(017)$ & $14.282(017)$ & $15.595(010)$ & $14.571(015)$ \\
\hline 5942.55 & $16.805(018)$ & $15.197(016)$ & $14.420(017)$ & $14.291(017)$ & $15.628(012)$ & \\
\hline 5943.57 & $16.836(016)$ & $15.219(013)$ & & $14.451(010)$ & $15.646(010)$ & $14.636(010)$ \\
\hline 5949.58 & 16.993(016) & $15.355(016)$ & $14.700(020)$ & $14.679(014)$ & $15.771(013)$ & $14.795(010)$ \\
\hline 5953.63 & 17.088(017) & 15.392(015) & $14.800(016)$ & $14.805(017)$ & $15.838(012)$ & $14.917(010)$ \\
\hline 5955.62 & $17.126(018)$ & $15.438(020)$ & & $14.808(017)$ & $15.864(013)$ & $14.960(016)$ \\
\hline 5957.63 & 17.184(019) & $15.488(016)$ & $14.940(016)$ & 14.988(009) & $15.920(010)$ & $15.020(008)$ \\
\hline 5962.60 & 17.302(018) & 15.591(016) & $15.102(014)$ & $15.140(015)$ & $15.988(010)$ & $15.134(010)$ \\
\hline 5963.66 & $17.301(021)$ & $15.610(016)$ & $15.155(015)$ & $15.137(018)$ & $15.996(010)$ & $15.163(008)$ \\
\hline 5965.66 & $17.338(023)$ & $15.626(019)$ & $15.208(015)$ & $15.269(016)$ & $16.029(011)$ & $\ldots$ \\
\hline 5967.65 & 17.382(023) & 15.662(012) & $15.299(016)$ & $15.318(015)$ & $16.068(010)$ & $15.276(008)$ \\
\hline 5969.61 & 17.502(019) & 15.676(019) & $15.396(022)$ & 15.368(018) & $16.088(010)$ & $15.320(011)$ \\
\hline 5972.64 & $17.576(020)$ & $15.714(020)$ & $15.547(030)$ & $15.486(016)$ & 16.117(011) & $15.368(011)$ \\
\hline 5981.61 & $17.811(021)$ & $15.850(020)$ & 15.797(022) & $15.775(016)$ & $16.255(011)$ & $15.596(009)$ \\
\hline 5985.57 & $17.910(020)$ & $15.921(020)$ & $15.972(029)$ & $15.909(018)$ & $16.309(011)$ & $15.699(011)$ \\
\hline 5991.61 & 17.998(040) & $16.075(014)$ & $16.140(013)$ & $16.078(011)$ & & \\
\hline 5994.57 & $18.113(042)$ & $16.146(023)$ & $16.268(018)$ & $16.198(016)$ & $16.487(014)$ & $15.954(012)$ \\
\hline 5997.55 & 18.195(027) & 16.138(019) & $16.376(022)$ & $16.265(019)$ & $16.514(012)$ & $16.003(012)$ \\
\hline 6001.57 & $18.400(027)$ & $16.222(018)$ & $16.490(021)$ & $16.359(021)$ & $16.578(012)$ & $16.091(012)$ \\
\hline 6004.58 & 18.491(033) & $16.276(017)$ & $\ldots$ & $16.465(018)$ & $16.619(012)$ & $16.151(009)$ \\
\hline 6006.57 & $18.525(033)$ & $16.305(021)$ & $\cdots$ & $16.542(017)$ & $16.666(013)$ & $16.222(013)$ \\
\hline 6008.50 & $18.556(028)$ & $16.368(025)$ & $\cdots$ & $16.574(020)$ & $16.696(015)$ & $16.235(019)$ \\
\hline 6013.53 & $\ldots$ & $16.452(018)$ & $\ldots$ & $16.625(023)$ & 16.783(013) & $16.388(012)$ \\
\hline 6016.53 & $\ldots$ & $16.504(015)$ & $\ldots$ & $16.710(025)$ & $16.860(013)$ & $16.438(014)$ \\
\hline 6019.51 & $18.845(049)$ & $16.567(017)$ & $\ldots$ & $16.750(017)$ & $16.937(013)$ & $16.463(010)$ \\
\hline 6024.53 & $\ldots$ & $\ldots$ & $\cdots$ & $16.909(020)$ & $\ldots$ & $\ldots$ \\
\hline 6026.50 & $\ldots$ & $16.690(015)$ & $\ldots$ & $16.928(015)$ & $\ldots$ & $\ldots$ \\
\hline 6031.51 & $\cdots$ & $\ldots$ & $\cdots$ & $\ldots$ & 17.048(016) & $\cdots$ \\
\hline 6032.50 & $\ldots$ & $16.889(016)$ & $\ldots$ & $\ldots$ & $\ldots$ & $\ldots$ \\
\hline
\end{tabular}


Table F.5. NIR photometry of SN 2007on and SN 2011 iv in the "natural" system.

\begin{tabular}{|c|c|c|c|c|}
\hline JD-2 450000 & $Y(\mathrm{mag})$ & $J$ (mag) & $H$ (mag) & Telescope $^{a}$ \\
\hline \multicolumn{5}{|c|}{ SN 2007on } \\
\hline 4410.73 & $14.419(018)$ & $14.353(016)$ & $14.273(017)$ & SWO \\
\hline 4410.74 & $14.450(017)$ & $14.312(021)$ & $14.259(016)$ & SWO \\
\hline 4412.73 & $13.835(017)$ & $13.739(010)$ & $13.742(014)$ & SWO \\
\hline 4412.74 & $13.850(013)$ & $13.723(014)$ & 13.737(009) & SWO \\
\hline 4415.75 & $13.324(014)$ & $13.217(011)$ & $\ldots$ & SWO \\
\hline 4415.76 & $13.331(013)$ & $13.216(014)$ & $\ldots$ & SWO \\
\hline 4421.75 & $13.435(026)$ & $13.304(041)$ & $13.365(046)$ & DUP \\
\hline 4421.76 & $13.438(027)$ & $13.312(041)$ & $13.345(046)$ & DUP \\
\hline 4422.84 & $13.481(025)$ & .. & ... & DUP \\
\hline 4422.85 & $13.511(026)$ & $\ldots$ & $\ldots$ & DUP \\
\hline 4423.76 & $13.546(026)$ & $13.533(040)$ & $\ldots$ & DUP \\
\hline 4423.76 & $13.559(027)$ & $\ldots$ & $\ldots$ & DUP \\
\hline 4424.73 & $13.572(012)$ & $13.627(013)$ & $13.473(011)$ & SWO \\
\hline 4424.77 & $13.589(014)$ & $13.649(013)$ & $13.456(013)$ & SWO \\
\hline 4425.77 & $13.672(025)$ & $13.850(040)$ & $\ldots$ & DUP \\
\hline 4425.78 & $13.672(025)$ & $13.890(042)$ & $\ldots$ & DUP \\
\hline 4426.79 & $13.696(026)$ & $13.989(041)$ & $13.542(046)$ & DUP \\
\hline 4426.80 & $13.720(026)$ & $14.016(041)$ & $13.528(046)$ & DUP \\
\hline 4427.73 & $13.704(026)$ & $14.125(041)$ & $13.535(046)$ & DUP \\
\hline 4427.73 & $13.676(026)$ & $14.125(041)$ & $13.527(046)$ & DUP \\
\hline 4428.73 & $13.684(025)$ & $14.220(040)$ & $13.517(046)$ & DUP \\
\hline 4428.73 & $13.710(026)$ & $14.207(040)$ & $13.539(046)$ & DUP \\
\hline 4431.73 & $13.534(014)$ & $14.229(014)$ & $13.434(012)$ & SWO \\
\hline 4431.74 & $13.524(013)$ & $14.243(014)$ & 13.451(009) & SWO \\
\hline 4432.68 & $13.483(012)$ & $14.239(016)$ & 13.391(014) & SWO \\
\hline 4432.69 & $13.475(016)$ & $14.232(015)$ & $13.411(012)$ & SWO \\
\hline 4437.72 & $13.185(012)$ & $14.040(011)$ & $13.425(012)$ & SWO \\
\hline 4437.73 & $13.252(014)$ & $14.035(014)$ & $13.431(008)$ & SWO \\
\hline 4439.70 & $13.181(017)$ & $14.062(012)$ & $13.542(015)$ & SWO \\
\hline 4439.70 & $13.210(016)$ & $14.065(015)$ & $13.559(008)$ & SWO \\
\hline 4442.72 & $13.400(017)$ & $14.429(015)$ & $13.840(013)$ & SWO \\
\hline 4442.73 & 13.417(018) & $14.442(015)$ & $13.851(010)$ & SWO \\
\hline 4444.79 & $13.562(007)$ & $14.712(012)$ & $14.026(013)$ & SWO \\
\hline 4444.80 & 13.563(008) & $14.725(011)$ & $14.021(013)$ & SWO \\
\hline 4446.69 & $13.719(013)$ & $14.928(015)$ & $14.179(015)$ & SWO \\
\hline 4446.70 & $13.762(016)$ & $14.922(016)$ & $14.186(013)$ & SWO \\
\hline 4448.67 & $13.839(011)$ & $15.141(013)$ & $14.300(014)$ & SWO \\
\hline 4448.67 & 13.841(008) & $15.149(014)$ & $14.331(010)$ & SWO \\
\hline 4454.66 & $14.237(013)$ & $15.733(015)$ & $14.678(016)$ & SWO \\
\hline 4454.67 & $14.255(013)$ & $15.690(021)$ & $14.682(014)$ & SWO \\
\hline 4456.66 & $14.388(026)$ & $\ldots$ & $14.786(046)$ & DUP \\
\hline 4457.73 & $14.459(026)$ & $\ldots$ & $14.845(046)$ & DUP \\
\hline 4457.73 & $14.482(026)$ & $\ldots$ & $14.883(047)$ & DUP \\
\hline 4461.69 & $14.689(010)$ & $16.315(020)$ & $15.014(013)$ & SWO \\
\hline 4461.70 & $14.700(012)$ & $16.299(021)$ & $15.053(011)$ & SWO \\
\hline 4463.66 & $14.826(013)$ & $16.449(017)$ & $15.134(014)$ & SWO \\
\hline 4463.67 & $14.844(014)$ & 16.491(019) & $15.178(013)$ & SWO \\
\hline 4468.67 & $15.142(027)$ & $\ldots$ & $15.431(046)$ & DUP \\
\hline 4468.67 & $15.138(026)$ & $\ldots$ & $15.430(046)$ & DUP \\
\hline 4471.66 & $15.270(012)$ & $16.952(025)$ & $15.540(018)$ & SWO \\
\hline 4471.67 & $15.303(015)$ & $17.063(033)$ & $15.542(022)$ & SWO \\
\hline 4477.62 & $15.574(010)$ & $17.497(051)$ & $15.872(021)$ & SWO \\
\hline 4477.63 & $15.581(010)$ & $17.545(051)$ & $15.874(024)$ & SWO \\
\hline 4485.64 & $15.927(013)$ & $17.972(081)$ & $16.338(031)$ & SWO \\
\hline 4485.65 & $15.919(013)$ & $18.049(078)$ & $16.323(035)$ & SWO \\
\hline 4491.60 & $16.140(011)$ & 18.146(093) & $16.656(035)$ & SWO \\
\hline 4491.61 & $16.166(013)$ & $18.313(103)$ & $16.623(044)$ & SWO \\
\hline
\end{tabular}

Notes. Values in parentheses are $1 \sigma$ measurement uncertainties in millimag. ${ }^{(a)}$ SWO and DUP correspond to the Swope and du Pont telescopes, respectively. NIR response functions are reported in Krisciunas et al., in prep. 
Table F.5. continued.

\begin{tabular}{ccccc}
\hline \hline JD-2 450 000 & $Y(\mathrm{mag})$ & $J(\mathrm{mag})$ & $H(\mathrm{mag})$ & Telescope $^{a}$ \\
\hline \multicolumn{5}{c}{ SN 2011iv } \\
\hline 5902.70 & $12.943(073)$ & $12.967(042)$ & $13.043(081)$ & DUP \\
5903.59 & $12.970(073)$ & $12.866(041)$ & $12.978(078)$ & DUP \\
5906.66 & $13.157(073)$ & $12.925(045)$ & $13.004(079)$ & DUP \\
5907.76 & $13.213(073)$ & $13.073(042)$ & $13.076(078)$ & DUP \\
5908.74 & $13.260(073)$ & $13.182(042)$ & $13.118(078)$ & DUP \\
5908.75 & $13.248(073)$ & $\ldots$ & $\ldots$ & DUP \\
5909.65 & $13.285(073)$ & $13.178(058)$ & $13.148(080)$ & DUP \\
5909.63 & $13.318(073)$ & $\ldots$ & $\ldots$ & DUP \\
5910.65 & $13.292(073)$ & $13.318(042)$ & $13.149(078)$ & DUP \\
5910.67 & $\ldots$ & $\ldots$ & $13.226(078)$ & DUP \\
5911.65 & $\ldots$ & $13.505(042)$ & $13.305(080)$ & DUP \\
5912.71 & $13.339(073)$ & $13.760(043)$ & $13.297(081)$ & DUP \\
5913.63 & $13.351(074)$ & $13.989(045)$ & $13.451(083)$ & DUP \\
5914.66 & $13.319(074)$ & $14.106(045)$ & $13.413(082)$ & DUP \\
5915.63 & $13.262(073)$ & $14.120(042)$ & $13.439(080)$ & DUP \\
5927.54 & $12.610(073)$ & $13.585(042)$ & $12.847(078)$ & DUP \\
5927.55 & $12.592(073)$ & $\ldots$ & $\ldots$ & DUP \\
5928.56 & $\ldots$ & $13.546(042)$ & $12.906(078)$ & DUP \\
5929.55 & $12.746(073)$ & $13.770(043)$ & $13.183(078)$ & DUP \\
5930.53 & $12.698(073)$ & $\ldots$ & $\ldots$ & DUP \\
5931.55 & $12.715(138)$ & $13.791(042)$ & $13.223(078)$ & DUP \\
5992.57 & $16.212(073)$ & $17.861(053)$ & $\ldots$ & DUP \\
6001.55 & $16.540(073)$ & $\ldots$ & $16.599(083)$ & DUP \\
6021.48 & $16.974(076)$ & $\ldots$ & $\ldots$ & DUP \\
6022.50 & $17.218(074)$ & $18.806(077)$ & $\ldots$ & DUP \\
\hline
\end{tabular}

Table F.6. Journal of spectroscopic observations for SN 2007 on.

\begin{tabular}{|c|c|c|c|c|}
\hline Date & JD-2450000 & Phase $^{a}$ & Telescope & Instrument \\
\hline \multicolumn{5}{|c|}{ Visual-wavelength spectroscopy } \\
\hline 2007 Nov. 11.3 & 4415.8 & -4.0 & du Pont & $\mathrm{WFCCD}^{b}$ \\
\hline 2007 Nov. 14.3 & 4418.8 & -1.0 & du Pont & $\mathrm{WFCCD}^{b}$ \\
\hline 2007 Nov. 17.2 & 4421.7 & +1.9 & Baade & $\mathrm{IMACS}^{b}$ \\
\hline 2007 Nov. 19.2 & 4423.7 & +3.9 & NTT & $\mathrm{EMMI}^{b}$ \\
\hline 2007 Nov. 25.2 & 4429.7 & +9.9 & Clay & $\operatorname{MagE}^{b}$ \\
\hline 2007 Nov. 27.2 & 4431.7 & +11.9 & NTT & $\mathrm{EMMI}^{c}$ \\
\hline 2007 Nov. 30.2 & 4434.7 & +14.9 & ESO-3P6 & $\mathrm{EFOSC}^{b}$ \\
\hline 2007 Dec. 03.2 & 4437.7 & +17.9 & du Pont & $\mathrm{WFCCD}^{b}$ \\
\hline 2007 Dec. 09.2 & 4443.7 & +23.9 & du Pont & $\mathrm{WFCCD}^{b}$ \\
\hline 2007 Dec. 10.2 & 4444.7 & +24.9 & du Pont & $\mathrm{WFCCD}^{b}$ \\
\hline 2007 Dec. 18.1 & 4452.6 & +32.8 & NTT & $\mathrm{EMMI}^{b}$ \\
\hline 2007 Dec. 27.3 & 4461.8 & +42.0 & Baade & IMACS $^{b}$ \\
\hline 2008 Jan. 03.1 & 4468.6 & +48.8 & ESO-3P6 & $\mathrm{EFOSC}^{b}$ \\
\hline 2008 Jan. 05.1 & 4470.6 & +50.8 & du Pont & $\mathrm{B} \& \mathrm{C}^{b}$ \\
\hline 2008 Jan. 10.1 & 4475.6 & +55.8 & du Pont & $\mathrm{B} \& \mathrm{C}^{b}$ \\
\hline 2008 Jan. 27.1 & 4492.6 & +72.8 & ESO-3P6 & $\mathrm{EFOSC}^{b}$ \\
\hline 2008 Feb. 28.2 & 4493.7 & +73.9 & NTT & $\mathrm{EMMI}^{c}$ \\
\hline 2008 Feb. 01.1 & 4497.6 & +77.8 & du Pont & $\mathrm{B} \& \mathrm{C}^{b}$ \\
\hline 2008 Feb. 13.1 & 4509.6 & +89.8 & NTT & $\mathrm{EMMI}^{b}$ \\
\hline 2008 Feb. 25.1 & 4521.6 & +101.8 & Clay & $\mathrm{LDSS}^{b}$ \\
\hline 2008 Aug. 27.3 & 4705.8 & +286.0 & Clay & $\operatorname{LDSS}^{b}$ \\
\hline 2008 Nov. 03.0 & 4773.5 & +353.7 & Gemini-South & ${\mathrm{GMOS}-\mathrm{S}^{d}}^{2}$ \\
\hline 2008 Nov. 30.0 & 4800.5 & +380.7 & Gemini-South & GMOS-S $^{d}$ \\
\hline \multicolumn{5}{|c|}{ NIR spectroscopy } \\
\hline 2007 Dec. 19.2 & 4453.7 & +33.9 & NTT & $\mathrm{SOFI}^{c}$ \\
\hline 2008 Feb. 14.1 & 4510.5 & +90.7 & NTT & $\mathrm{SOFI}^{c}$ \\
\hline
\end{tabular}

Notes. ${ }^{(a)}$ Days relative to $T(B)_{\max }$, i.e. JD-2 454 419.8. ${ }^{(b)}$ Published by Folatelli et al. (2013). ${ }^{(c)}$ This work, see Sect. 2. ${ }^{(d)}$ Previously included in analysis by Maeda et al. (2010). We note that the $+380 \mathrm{~d}$ spectrum now includes a vastly improved wavelength calibration at the blue end of the spectrum. 
Table F.7. Journal of spectroscopic observations for SN 2011iv.

\begin{tabular}{|c|c|c|c|c|}
\hline Date & JD-2450 000 & Phase $^{a}$ & Telescope & Instrument \\
\hline \multicolumn{5}{|c|}{ UV - Visual-wavelength spectroscopy } \\
\hline 2011 Dec. 05.4 & 5901.0 & -5.1 & SWIFT & UVOT \\
\hline 2011 Dec. 06.6 & 5902.1 & -4.0 & SWIFT & UVOT \\
\hline 2011 Dec. 07.6 & 5903.1 & -3.0 & SWIFT & UVOT \\
\hline 2011 Dec. 09.6 & 5905.1 & -1.0 & SWIFT & UVOT \\
\hline 2011 Dec. 11.7 & 5907.2 & +1.1 & SWIFT & UVOT \\
\hline 2011 Dec. 11.0 & 5906.5 & +0.4 & HST & STIS \\
\hline 2011 Dec. 15.0 & 5910.5 & +4.4 & HST & STIS \\
\hline 2011 Dec. 20.0 & 5915.5 & +9.4 & HST & STIS \\
\hline 2011 Dec. 24.0 & 5919.5 & +13.4 & HST & STIS \\
\hline 2011 Dec. 28.0 & 5923.5 & +17.4 & HST & STIS \\
\hline 2012 Jan. 01.0 & 5927.5 & +21.4 & HST & STIS \\
\hline 2012 Jan. 09.0 & 5935.5 & +29.4 & HST & STIS \\
\hline \multicolumn{5}{|c|}{ Visual-wavelength spectroscopy } \\
\hline 2011 Dec. 04.0 & 5899.5 & -6.6 & Gemini-South & GMOS-S \\
\hline 2011 Dec. 04.0 & 5899.5 & -6.6 & du Pont & $\mathrm{B} \& \mathrm{C}$ \\
\hline 2011 Dec. 05.0 & 5900.6 & -5.5 & du Pont & $\mathrm{B} \& \mathrm{C}$ \\
\hline 2011 Dec. 06.0 & 5901.6 & -4.5 & du Pont & $\mathrm{B} \& \mathrm{C}$ \\
\hline 2011 Dec. 18.0 & 5913.5 & +7.4 & WHT & ISIS \\
\hline 2011 Dec. 18.1 & 5913.8 & +7.7 & SOAR & Goodman \\
\hline 2011 Dec. 19.0 & 5914.5 & +8.4 & WHT & ISIS \\
\hline 2011 Dec. 19.2 & 5914.7 & +8.6 & NTT & EFOSC \\
\hline 2011 Dec. 20.0 & 5915.5 & +9.4 & WHT & ISIS \\
\hline 2011 Dec. 21.0 & 5916.5 & +10.4 & WHT & ISIS \\
\hline 2011 Dec. 21.1 & 5916.6 & +10.5 & du Pont & WFCCD \\
\hline 2011 Dec. 27.0 & 5922.0 & +15.9 & NOT & ALFOSC \\
\hline 2011 Dec. 31.1 & 5926.6 & +20.5 & du Pont & WFCCD \\
\hline 2011 Dec. 31.2 & 5926.8 & +20.7 & SOAR & Goodman \\
\hline 2011 Jan. 10.0 & 5936.0 & +29.9 & NOT & ALFOSC \\
\hline 2012 Jan. 19.0 & 5945.6 & +39.5 & du Pont & WFCCD \\
\hline 2012 Jan. 22.2 & 5948.7 & +42.6 & NTT & EFOSC \\
\hline 2012 Feb. 04.1 & 5961.5 & +55.4 & Clay & MIKE \\
\hline 2012 Mar. 02.1 & 5988.5 & +82.4 & Clay & LDSS3 \\
\hline 2012 Apr. 30.9 & 6048.5 & +142.4 & du Pont & WFCCD \\
\hline 2012 Aug. 10.4 & 6150.0 & +243.9 & du Pont & WFCCD \\
\hline 2012 Aug. 27.3 & 6166.8 & +260.7 & NTT & EFOSC \\
\hline 2012 Sept. 11.3 & 6181.8 & +275.7 & du Pont & WFCCD \\
\hline \multicolumn{5}{|c|}{ NIR spectrscopy } \\
\hline 2011 Dec. 09.0 & 5904.5 & -1.6 & Baade & FIRE \\
\hline 2011 Dec. 11.0 & 5906.5 & +0.4 & Baade & FIRE \\
\hline 2011 Dec. 11.0 & 5906.7 & +0.6 & Baade & FIRE \\
\hline 2011 Dec. 14.0 & 5909.5 & +3.4 & VLT & ISAAC \\
\hline 2011 Dec. 16.2 & 5911.7 & +5.6 & Baade & FIRE \\
\hline 2011 Dec. 18.2 & 5913.7 & +7.6 & Baade & FIRE \\
\hline 2011 Dec. 20.2 & 5915.7 & +9.6 & NTT & SOFI \\
\hline 2011 Dec. 21.2 & 5916.7 & +10.6 & Baade & FIRE \\
\hline 2011 Dec. 29.1 & 5924.6 & +18.5 & VLT & ISAAC \\
\hline 2012 Jan. 07.1 & 5933.6 & +27.5 & Baade & FIRE \\
\hline 2012 Jan. 15.1 & 5941.6 & +35.5 & Baade & FIRE \\
\hline 2012 Jan. 19.2 & 5945.7 & +39.6 & Baade & FIRE \\
\hline 2012 Feb. 03.1 & 5960.6 & +54.5 & Baade & FIRE \\
\hline 2012 Feb. 16.1 & 5973.6 & +67.5 & NTT & SOFI \\
\hline 2012 Mar. 03.1 & 5989.6 & +83.5 & Baade & FIRE \\
\hline 2012 Apr. 30.0 & 6047.5 & +141.4 & Baade & FIRE \\
\hline
\end{tabular}

Notes. ${ }^{(a)}$ Days relative to $T(B)_{\max }$, i.e. JD-2 455906.1 . 
Table F.8. SNooPy UVOIR light curve of SN 2007on.

\begin{tabular}{|c|c|c|}
\hline Phase (day) ${ }^{a}$ & $L_{\text {bol }}\left(\mathrm{erg} \mathrm{s}^{-1}\right)^{b}$ & $\bar{U}^{\prime}$ ncertainty $\left(\mathrm{erg} \mathrm{s}^{-1}\right)^{c}$ \\
\hline-7.87 & $3.472 \times 10^{47}$ & $3.145 \times 10^{45}$ \\
\hline-6.80 & $2.308 \times 10^{42}$ & $3.834 \times 10^{39}$ \\
\hline-5.88 & $2.988 \times 10^{42}$ & $2.516 \times 10^{40}$ \\
\hline-4.78 & $3.735 \times 10^{42}$ & $1.676 \times 10^{40}$ \\
\hline-3.21 & $4.756 \times 10^{42}$ & $8.906 \times 10^{40}$ \\
\hline-1.12 & $5.600 \times 10^{42}$ & $3.607 \times 10^{40}$ \\
\hline+0.12 & $5.726 \times 10^{42}$ & $7.056 \times 10^{40}$ \\
\hline+1.36 & $5.703 \times 10^{42}$ & $1.198 \times 10^{40}$ \\
\hline+2.85 & $5.422 \times 10^{42}$ & $1.051 \times 10^{40}$ \\
\hline+4.07 & $5.147 \times 10^{42}$ & $4.063 \times 10^{40}$ \\
\hline+4.84 & $4.779 \times 10^{42}$ & $2.826 \times 10^{40}$ \\
\hline+6.04 & $4.454 \times 10^{42}$ & $5.467 \times 10^{40}$ \\
\hline+7.03 & $4.117 \times 10^{42}$ & $3.410 \times 10^{40}$ \\
\hline+7.98 & $3.794 \times 10^{42}$ & $1.229 \times 10^{40}$ \\
\hline+8.71 & $3.523 \times 10^{42}$ & $2.079 \times 10^{40}$ \\
\hline+10.03 & $3.253 \times 10^{42}$ & $8.012 \times 10^{39}$ \\
\hline+10.99 & $2.974 \times 10^{42}$ & $1.409 \times 10^{40}$ \\
\hline+11.96 & $2.775 \times 10^{42}$ & $1.575 \times 10^{40}$ \\
\hline+12.69 & $2.665 \times 10^{42}$ & $9.730 \times 10^{39}$ \\
\hline+13.94 & $2.490 \times 10^{42}$ & $1.162 \times 10^{40}$ \\
\hline+14.87 & $2.389 \times 10^{42}$ & $5.700 \times 10^{39}$ \\
\hline+16.92 & $2.231 \times 10^{42}$ & $7.396 \times 10^{39}$ \\
\hline+17.76 & $2.170 \times 10^{42}$ & $6.581 \times 10^{39}$ \\
\hline+18.95 & $2.056 \times 10^{42}$ & $1.178 \times 10^{40}$ \\
\hline+20.91 & $1.851 \times 10^{42}$ & $2.629 \times 10^{40}$ \\
\hline+21.92 & $1.710 \times 10^{42}$ & $2.391 \times 10^{40}$ \\
\hline+23.92 & $1.446 \times 10^{42}$ & $1.966 \times 10^{40}$ \\
\hline+24.93 & $1.332 \times 10^{42}$ & $1.339 \times 10^{40}$ \\
\hline+25.98 & $1.229 \times 10^{42}$ & $6.977 \times 10^{39}$ \\
\hline+26.92 & $1.145 \times 10^{42}$ & $5.039 \times 10^{39}$ \\
\hline+27.86 & $1.058 \times 10^{42}$ & $3.160 \times 10^{39}$ \\
\hline+29.83 & $9.329 \times 10^{41}$ & $6.400 \times 10^{39}$ \\
\hline $\begin{array}{r}127.05 \\
+30.87\end{array}$ & $8.778 \times 10^{41}$ & $4.627 \times 10^{39}$ \\
\hline+32.26 & $8.194 \times 10^{41}$ & $6.796 \times 10^{39}$ \\
\hline+34.78 & $7.270 \times 10^{41}$ & $1.390 \times 10^{39}$ \\
\hline $\begin{array}{l}+34.10 \\
+35.78\end{array}$ & $6.929 \times 10^{41}$ & $2.545 \times 10^{39}$ \\
\hline $\begin{array}{l}+53.10 \\
+36.33\end{array}$ & $6.682 \times 10^{41}$ & $1.823 \times 10^{39}$ \\
\hline $\begin{array}{l}+30.53 \\
+37.78\end{array}$ & $6.379 \times 10^{41}$ & $1.755 \times 10^{39}$ \\
\hline $\begin{array}{r}51.10 \\
+38.74\end{array}$ & $6.131 \times 10^{41}$ & $2.644 \times 10^{39}$ \\
\hline+39.80 & $5.876 \times 10^{41}$ & $3.588 \times 10^{39}$ \\
\hline+40.91 & $5.640 \times 10^{41}$ & $3.475 \times 10^{39}$ \\
\hline+42.77 & $5.244 \times 10^{41}$ & $3.100 \times 10^{39}$ \\
\hline+43.39 & $5.041 \times 10^{41}$ & $3.261 \times 10^{39}$ \\
\hline+44.72 & $4.822 \times 10^{41}$ & $2.960 \times 10^{39}$ \\
\hline+48.68 & $4.111 \times 10^{41}$ & $1.128 \times 10^{39}$ \\
\hline+49.68 & $3.958 \times 10^{41}$ & $4.050 \times 10^{39}$ \\
\hline $\begin{array}{l}+50.84 \\
\end{array}$ & $3.828 \times 10^{41}$ & $1.457 \times 10^{39}$ \\
\hline+51.63 & $3.688 \times 10^{41}$ & $2.102 \times 10^{39}$ \\
\hline+52.68 & $3.558 \times 10^{41}$ & $3.110 \times 10^{38}$ \\
\hline+53.63 & $3.435 \times 10^{41}$ & $1.297 \times 10^{39}$ \\
\hline+56.62 & $3.127 \times 10^{41}$ & $1.760 \times 10^{39}$ \\
\hline $\begin{array}{r}75.64 \\
+57.64\end{array}$ & $3.037 \times 10^{41}$ & $3.112 \times 10^{39}$ \\
\hline+58.59 & $2.946 \times 10^{41}$ & $3.324 \times 10^{39}$ \\
\hline+65.54 & $2.412 \times 10^{41}$ & $1.422 \times 10^{39}$ \\
\hline+66.56 & $2.294 \times 10^{41}$ & $1.818 \times 10^{39}$ \\
\hline+69.49 & $2.090 \times 10^{41}$ & $2.981 \times 10^{38}$ \\
\hline
\end{tabular}

Notes. ${ }^{(a)}$ Days relative to $L_{\max }$, i.e. JD-2 454421.6. ${ }^{(b)}$ Assuming $\mu=31.27$ mag to set the absolute flux scale. ${ }^{(c)}$ Uncertainties represent SNooPy fitting errors obtained via Monte Carlos simulations, and do not include the error in the adopted distance.
Table F.9. SNooPy UVOIR light curve of SN 2011iv.

\begin{tabular}{|c|c|c|}
\hline Phase (day) ${ }^{a}$ & $L_{\mathrm{bol}}\left(\mathrm{erg} \mathrm{s}^{-1}\right)^{b}$ & Uncertainty $\left(\mathrm{erg} \mathrm{s}^{-1}\right)^{c}$ \\
\hline-2.60 & $9.327 \times 10^{42}$ & $3.920 \times 10^{40}$ \\
\hline-1.78 & $9.224 \times 10^{42}$ & $2.312 \times 10^{40}$ \\
\hline-0.99 & $9.309 \times 10^{42}$ & $1.307 \times 10^{40}$ \\
\hline+0.40 & $9.186 \times 10^{42}$ & $2.010 \times 10^{39}$ \\
\hline+1.35 & $8.996 \times 10^{42}$ & $1.005 \times 10^{39}$ \\
\hline+2.29 & $8.891 \times 10^{42}$ & $3.116 \times 10^{40}$ \\
\hline+3.38 & $8.430 \times 10^{42}$ & $4.221 \times 10^{40}$ \\
\hline+4.30 & $8.045 \times 10^{42}$ & $6.834 \times 10^{40}$ \\
\hline+4.98 & $7.731 \times 10^{42}$ & $7.739 \times 10^{40}$ \\
\hline+6.30 & $6.972 \times 10^{42}$ & $7.638 \times 10^{40}$ \\
\hline+7.16 & $6.574 \times 10^{42}$ & $6.734 \times 10^{40}$ \\
\hline+8.28 & $5.978 \times 10^{42}$ & $5.829 \times 10^{40}$ \\
\hline+8.95 & $5.662 \times 10^{42}$ & $7.638 \times 10^{40}$ \\
\hline+10.22 & $5.177 \times 10^{42}$ & $3.518 \times 10^{40}$ \\
\hline+11.26 & $4.735 \times 10^{42}$ & $3.518 \times 10^{40}$ \\
\hline+12.25 & $4.445 \times 10^{42}$ & $1.910 \times 10^{40}$ \\
\hline+13.22 & $4.242 \times 10^{42}$ & $1.809 \times 10^{40}$ \\
\hline+15.41 & $3.875 \times 10^{42}$ & $2.010 \times 10^{40}$ \\
\hline+17.22 & $3.691 \times 10^{42}$ & $2.111 \times 10^{40}$ \\
\hline+18.17 & $3.593 \times 10^{42}$ & $2.915 \times 10^{40}$ \\
\hline+19.17 & $3.437 \times 10^{42}$ & $4.824 \times 10^{40}$ \\
\hline+20.14 & $3.318 \times 10^{42}$ & $4.020 \times 10^{40}$ \\
\hline+21.07 & $3.170 \times 10^{42}$ & $3.618 \times 10^{40}$ \\
\hline+22.10 & $2.990 \times 10^{42}$ & $2.714 \times 10^{40}$ \\
\hline+23.11 & $2.759 \times 10^{42}$ & $9.347 \times 10^{40}$ \\
\hline+24.09 & $2.633 \times 10^{42}$ & $9.045 \times 10^{40}$ \\
\hline+25.08 & $2.368 \times 10^{42}$ & $1.266 \times 10^{41}$ \\
\hline+26.09 & $2.240 \times 10^{42}$ & $6.935 \times 10^{40}$ \\
\hline+27.11 & $2.072 \times 10^{42}$ & $4.322 \times 10^{40}$ \\
\hline+28.10 & $1.957 \times 10^{42}$ & $4.020 \times 10^{40}$ \\
\hline+29.09 & $1.832 \times 10^{42}$ & $3.015 \times 10^{40}$ \\
\hline+29.72 & $1.726 \times 10^{42}$ & $3.015 \times 10^{40}$ \\
\hline+32.05 & $1.479 \times 10^{42}$ & $1.709 \times 10^{40}$ \\
\hline+33.07 & $1.432 \times 10^{42}$ & $2.211 \times 10^{40}$ \\
\hline+34.04 & $1.366 \times 10^{42}$ & $1.608 \times 10^{40}$ \\
\hline+35.03 & $1.273 \times 10^{42}$ & $1.206 \times 10^{40}$ \\
\hline+36.03 & $1.227 \times 10^{42}$ & $1.809 \times 10^{40}$ \\
\hline+37.01 & $1.186 \times 10^{42}$ & $1.809 \times 10^{40}$ \\
\hline+38.01 & $1.100 \times 10^{42}$ & $1.407 \times 10^{40}$ \\
\hline+43.99 & $8.850 \times 10^{41}$ & $1.508 \times 10^{40}$ \\
\hline+48.01 & $7.733 \times 10^{41}$ & $8.442 \times 10^{39}$ \\
\hline+50.00 & $7.357 \times 10^{41}$ & $1.809 \times 10^{39}$ \\
\hline+51.99 & $6.680 \times 10^{41}$ & $7.035 \times 10^{39}$ \\
\hline+56.93 & $5.841 \times 10^{41}$ & $6.030 \times 10^{38}$ \\
\hline+57.98 & $5.739 \times 10^{41}$ & $2.513 \times 10^{39}$ \\
\hline+59.97 & $5.387 \times 10^{41}$ & $2.010 \times 10^{38}$ \\
\hline+61.94 & $5.108 \times 10^{41}$ & $1.106 \times 10^{39}$ \\
\hline+63.89 & $4.831 \times 10^{41}$ & $8.040 \times 10^{38}$ \\
\hline+66.90 & $4.451 \times 10^{41}$ & $6.030 \times 10^{38}$ \\
\hline+75.82 & $3.552 \times 10^{41}$ & $2.010 \times 10^{39}$ \\
\hline+79.75 & $3.200 \times 10^{41}$ & $2.613 \times 10^{39}$ \\
\hline+85.76 & $2.768 \times 10^{41}$ & $1.608 \times 10^{39}$ \\
\hline+86.70 & $2.690 \times 10^{41}$ & $6.030 \times 10^{38}$ \\
\hline+88.69 & $2.538 \times 10^{41}$ & $1.005 \times 10^{38}$ \\
\hline+91.66 & $2.394 \times 10^{41}$ & $1.005 \times 10^{39}$ \\
\hline
\end{tabular}

Notes. ${ }^{(a)}$ Days relative to $L_{\max }$, i.e. JD-2 $455907.6 .{ }^{(b)}$ Assuming $\mu=31.27$ mag to set the absolute flux scale. ${ }^{(c)}$ Uncertainties represent SNooPy fitting errors obtained via Monte Carlos simulations, and do not include the error in the adopted distance. 
C. Gall et al.: CSP observations of SN 2007on and SN 2011iv

Table F.10. UVOIR light curve of SN 2011 iv derived from spectra.

\begin{tabular}{cccc}
\hline \hline${\text { Phase }(\text { day })^{a}}$ & $L_{\text {bol }}\left(\mathrm{erg} \mathrm{s}^{-1}\right)^{b}$ & Wavelength range $(\AA)$ & Instrument \\
\hline-0.12 & $8.813 \times 10^{42}$ & $1615.50-24776.80$ & STIS, FIRE \\
+3.88 & $7.068 \times 10^{42}$ & $1626.50-24776.80$ & STIS, FIRE \\
+6.88 & $5.806 \times 10^{42}$ & $3347.32-24776.80$ & ISIS, FIRE \\
+7.88 & $5.481 \times 10^{42}$ & $3331.15-24776.80$ & ISIS, FIRE \\
+8.88 & $5.168 \times 10^{42}$ & $1620.00-24776.80$ & STIS, FIRE \\
+8.88 & $5.055 \times 10^{42}$ & $3293.07-24776.80$ & ISIS, FIRE \\
+9.88 & $4.632 \times 10^{42}$ & $3296.12-24776.80$ & ISIS, FIRE \\
+10.13 & $4.447 \times 10^{42}$ & $3698.07-24776.80$ & WFCCD, FIRE \\
+12.88 & $3.981 \times 10^{42}$ & $1624.50-24776.80$ & STIS, FIRE \\
+16.88 & $3.380 \times 10^{42}$ & $1624.50-24776.80$ & STIS, FIRE \\
+20.13 & $3.011 \times 10^{42}$ & $3632.00-24776.80$ & WFCCD, FIRE \\
+20.88 & $3.033 \times 10^{42}$ & $1627.50-24776.80$ & STIS, FIRE \\
+28.88 & $1.856 \times 10^{42}$ & $1635.00-24776.80$ & STIS, FIRE \\
+38.88 & $1.019 \times 10^{42}$ & $3628.00-24776.80$ & WFCCD, FIRE \\
\hline
\end{tabular}

Notes. ${ }^{(a)}$ Days relative to $L_{\max }$, i.e. JD-2 455 907.6. ${ }^{(b)}$ Assuming $\mu=31.27$ mag to set the absolute flux scale. 